\title{
Turbulence Modulation in Gas-Particle Flows: A Comparison of SElECted Models
}

\author{
by
}

Sarah M. Hodgson

\begin{abstract}
A thesis submitted in conformity with the requirements for the degree of Master of Applied Science Graduate Department of Mechanical and Industrial Engineering University of Toronto
\end{abstract}

Copyright (C) 1999 by Sarah M. Hodgson 
National Library

of Canada

Acquisitions and

Bibliographic Services

395 Wellington Street

Otrawa ON K1A ONA

Canada
Bibliothèque nationale du Canada

Acquisitions et services bibliographiques

395. ne Wellington Otawa ON K1A ONA Canada
The author has granted a nonexclusive licence allowing the National Library of Canada to reproduce, loan, distribute or sell copies of this thesis in microform, paper or electronic formats.

The author retains ownership of the copyright in this thesis. Neither the thesis nor substantial extracts from it may be printed or otherwise reproduced without the author's permission.
L'auteur a accordé une licence non exclusive permettant à la Bibliothèque nationale du Canada de reproduire, prêter, distribuer ou vendre des copies de cette thèse sous la forme de microfiche/film, de reproduction sur papier ou sur format électronique.

L'auteur conserve la propriété du droit d'auteur qui protège cette thèse. $\mathrm{Ni}$ la thèse ni des extraits substantiels de celle-ci ne doivent être imprimés ou autrement reproduits sans son autorisation. 


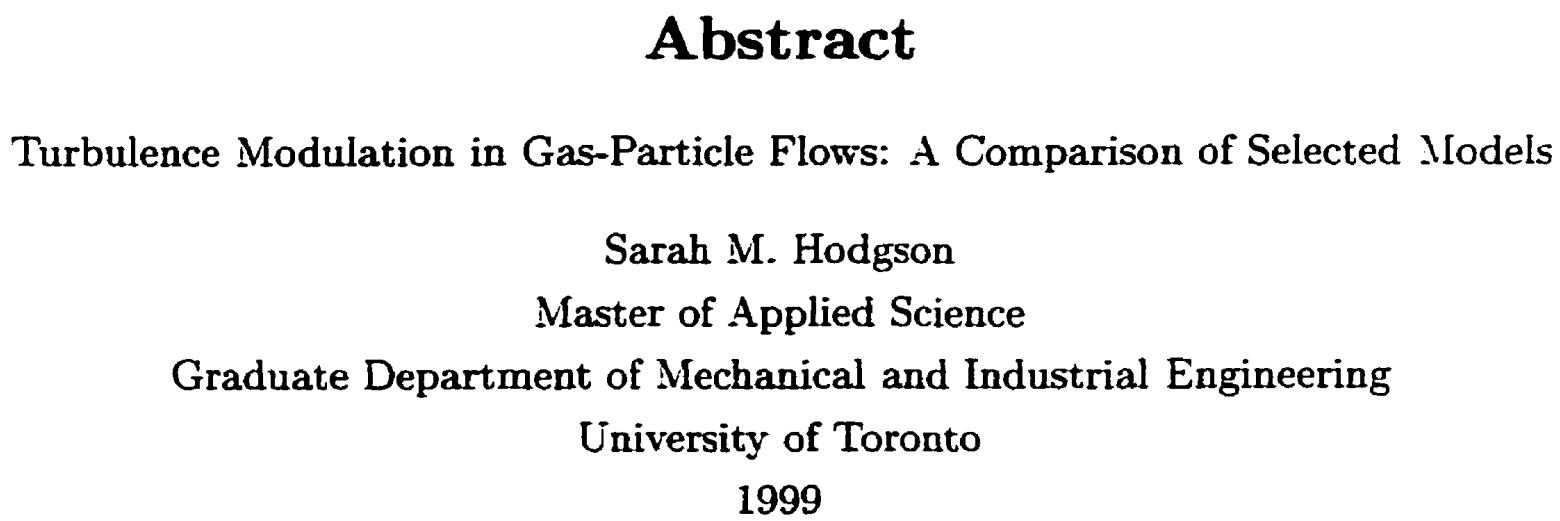

The problem of turbulence modulation, the process whereby the gas-phase turbulence is modified by the presence of particles, is investigated. Experimental trends are examined and parameters affecting turbulence modulation and the mechanisms by which turbulence modulation occurs are identified. A new model that accounts for the crossing trajectory effect is presented. This model and the turbulence modulation models of Chen and Wood [4], Tu and Fletcher [45], and Mostafa and Mongia [30] are investigated using the TASCflow CFD code. The models are compared with the experimental results of Tsuji et al [44]. The model of $\mathrm{Tu}$ and Fletcher is not able to reproduce either general experimental trends or the experimental results, while the other three models can predict the general experimental trends but cannot reproduce the experimental results. Analysis shows that the turbulent viscosity, $\mu_{t}$, plays an important role in modifying the turbulence intensity profiles. The new model was not able to capture the crossing trajectory effect for the flow considered. 


\section{Acknowledgements}

First and foremost I would like to thank my supervisor, Prof. Marilyn Lightstone. for the support and guidance she has provided. Her commitment to teaching and research has been inspiring.

I would also like to thank Eleanor Hennick, Ross Rock and everyone in the CFD lab for helping me out when I got stuck.

Finally a great big thanks to Katharine, James, Mat, Anna-Marie, Kirsten. Celia. Matthew and Andrea for keeping me sane and listening to me even when they didn $t$ know what I was talking about. 


\section{Dedication}

In memory of my mother, Marguerite Hodgson. 


\section{Contents}

List of Tables $\quad$ viii

List of Figures $\quad$ ix

$\begin{array}{ll}\text { Nomenclature } & \text { xii }\end{array}$

1 Introduction $\quad 1$

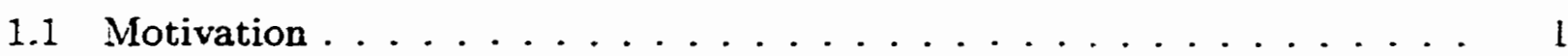

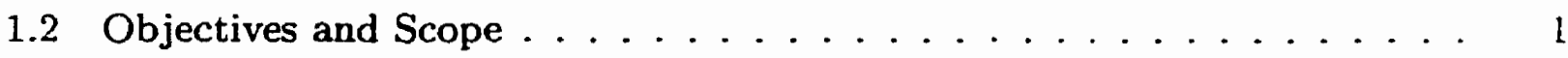

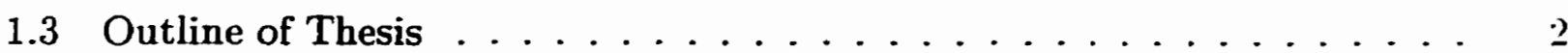

2 Background 4

2.1 Experimental Trends ...................... . 4

2.1.1 Parameters Affecting Turbulence Modulation . . . . . . . . . . . .

2.1.2 Mechanisms of Modulation . . . . . . . . . . . . . . 6

2.1.3 Mean Gas-Phase .................. . . 8

2.2 Physics of the Flow . . . . . . . . . . . . . . . . . 8

2.2.1 Particle Equation of Motion ............... . . s

2.2.2 Conservation Equations for the Gas-Phase . . . . . . . . . . 1:3

2.2.3 Turbulent Kinetic Energy ................ It

2.2.4 Turbulent Dissipation Equation . . . . . . . . . . . . 14

2.2.5 Coupling Between the Phases .............. . . . . . . . . .

2.3 Review of Existing Models . . . . . . . . . . . . . . . . IT

2.3.1 Lagrangian versus Eulerian Formulation ............ I

2.3.2 Particulate-Phase Model . . . . . . . . . . . . 19

2.3.3 Gas-Phase Model ... . . . . . . . . . . . . . 20

2.3.4 Turbulence Modulation Models . . . . . . . . . . . 22

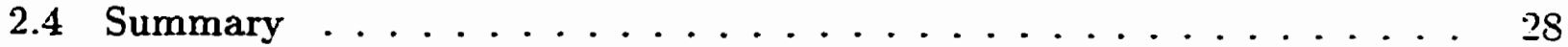


3 The Model of Lightstone and Hodgson $\quad 31$

3.1 Motivation . . . . . . . . . . . . . . . . . . . 31

3.2 The Model of Lightstone and Hodgson . . . . . . . . . . . . . . . . . . . . . . . . . .

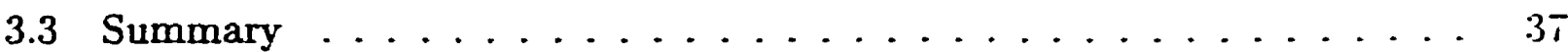

4 Numerical Solution $\quad 38$

4.1 Introduction . . . . . . . . . . . . . . . . . 38

4.2 Numerical Solution to the Governing Equations for the Gas-Phase . . . $\quad 38$

4.2.1 Grid Specification . . . . . . . . . . . . . . . . 38

4.2 .2 Mass and Momentum Equations . . . . . . . . . . . . 39

$4.2 .3 \quad k$ and $\epsilon$ Equations . . . . . . . . . . . . . . . 41

4.2.4 Evaluation of the Discretized Equations _........... . 42

4.2 .5 Boundary Conditions . . . . . . . . . . . . . 4.

4.2.6 Pressure-Velocity Coupling . . . . . . . . . . . . . 48

4.2 .7 Linear Solver .................... . . . 48

4.3 Numerical Solution of the Particulate-Phase . . . . . . . . . . . . 49

4.3.1 Implementation of the SSF Model . . . . . . . . . . . . . 49

4.3 .2 Particle Injection . . . . . . . . . . . . . . . j0

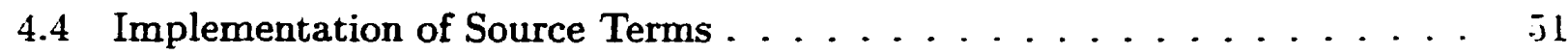

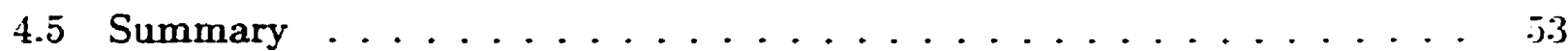

5 Validation $\quad \mathbf{5 4}$

5.1 Laminar Pipe Flow . . . . . . . . . . . . . . . . . j4

5.2 Turbulent Pipe Flow . . . . . . . . . . . . . . . . . . 6

5.3 Particle Dispersion . . . . . . . . . . . . . . . . . 64

5.3.1 Relevance to Taylor's Analysis . . . . . . . . . . . . . . . 68

5.4 Turbulent Source Term Implementation . . . . . . . . . . . . . . . . 69

5.5 Momentum Source Term Implementation . . . . . . . . . . . . . . il

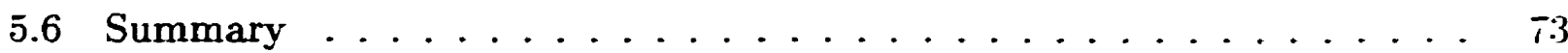

6 Results $\quad 75$

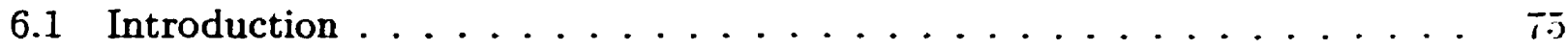

6.2 The Experiment of Tsuji et al, 1983 . . . . . . . . . . . . T.j

6.3 Turbulence Modulation Models . . . . . . . . . . . . . . . T

6.4 Simulation .............................

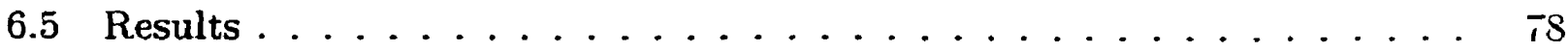

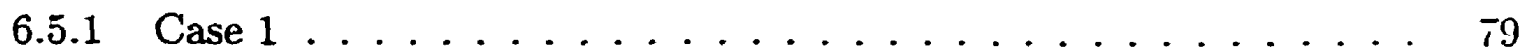




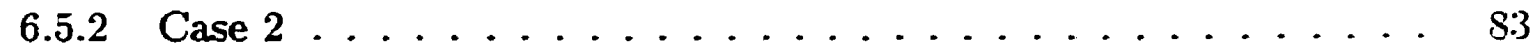

6.5 .3 Case $3 \ldots \ldots \ldots \ldots \ldots \ldots \ldots \ldots \ldots \ldots \ldots$

6.5.4 Turbulence Modulation Trend Predictions . . . . . . . . . 90

6.6 Analysis . . . . . . . . . . . . . . . . . . 04

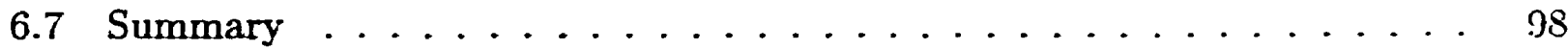

7 Conclusion $\quad 100$

7.1 Summary and Conclusions . . . . . . . . . . . . . 100

7.2 Recommendations for Future Work . . . . . . . . . . . . . 102

A Derivation of Turbulent Kinetic Energy Equation $\quad 104$

$\begin{array}{ll}\text { B Analysis of Momentum Source Term } & 106\end{array}$

$\begin{array}{ll}\text { Bibliography } & 107\end{array}$ 


\section{List of Tables}

2.1 Experimental Results $\ldots \ldots \ldots \ldots \ldots$

2.2 Summary of Models Reviewed . . . . . . . . . . . . . . . 29

5.1 Summary of Sensitivity Analysis . . . . . . . . . . . . 64

6.1 Turbulence Modulation Models to be Investigated . . . . . . . . it

6.2 Particle test cases . . . . . . . . . . . . . . . . . is

6.3 Summary of Handchecks: Case $1 \ldots \ldots \ldots \ldots . \ldots . \ldots . \ldots . \ldots$ 


\section{List of Figures}

3.1 Particle and Fluid Trajectories $\ldots \ldots \ldots \ldots \ldots \ldots \ldots$

4.1 Flux element with 7 of the 8 nodes visible . . . . . . . . . . 39

4.2 Boundary and Interior Control Volumes . . . . . . . . . . 40

4.3 One-Dimensional flow moving from point $\mathrm{P}$ to $\mathrm{E} \ldots \ldots \ldots$

4.4 Near-Wall Region $\ldots \ldots \ldots \ldots . \ldots . \ldots . \ldots$

5.1 Computational Grid (truncated in k-direction) . . . . . . . . . . . j

5.2 Axial velocity, analytical solution and code prediction using wedge-shaped

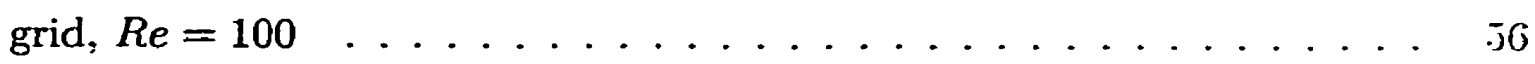

5.3 Mean velocity: Prediction of analytical solution and experimental data of Tsuji et al.[44], $R e \approx 23000 \ldots \ldots \ldots \ldots \ldots \ldots$.js

5.4 Turbulence intensity: Prediction of experimental data of Tsuji et al.[4t].

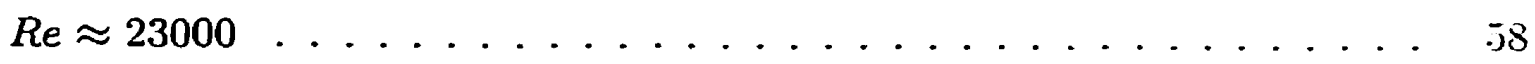

5.5 Mean velocity: Prediction using coarse $(15 \times 3 \times 1500)$ and fine $(30 \times 3 \times 1700)$ grids of analytical solution and experimental data of Tsuji et al.[44], Re $\approx$

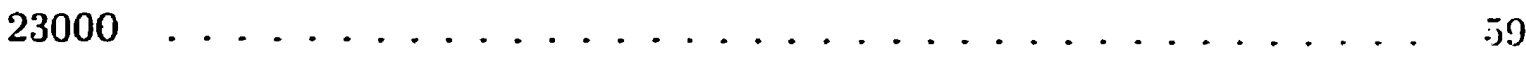

5.6 Turbulence intensity: Prediction using coarse $(15 \times 3 \times 1500)$ and fine $(30 \times 3 \times 1700)$ grids of experimental data of Tsuji et al.[44], $R e \approx 23000 \quad \ldots . . . \quad .59$

5.7 Mean velocity: Sensitivity of prediction to changes in inlet values of $T_{i}$. Prediction of analytical solution and experimental data of Tsuji et al.[4t].

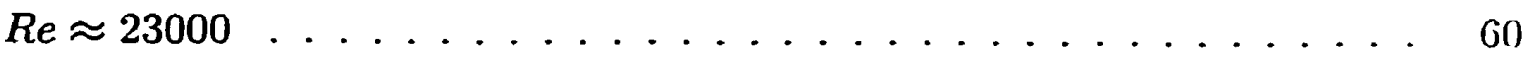

5.8 Turbulence intensity: Sensitivity of prediction to changes in inlet values of $T_{i}$. Prediction of experimental data of Tsuji et al.[44], $R e \approx 23000$.

5.9 Mean velocity: Sensitivity of prediction to changes in inlet values of $L_{\epsilon}$. Prediction of analytical solution and experimental data of Tsuji et al. [tt].

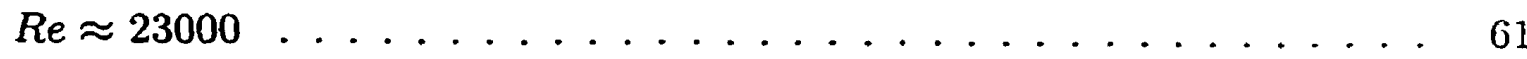

5.10 Turbulence intensity: Sensitivity of prediction to changes in inlet values of $L_{\epsilon}$. Prediction of experimental data of Tsuji et al.[44], $R e \approx 23000$. . 
5.11 Mean velocity: Sensitivity of prediction to changes in $y^{+}$. Prediction of analytical solution and experimental data of Tsuji et al.[44]: $R e \approx 23000$

5.12 Turbulence intensity: Sensitivity of prediction to changes in $y^{\dagger}$. Prediction of experimental data of Tsuji et al.[44], $R e \approx 23000 \ldots . . . . .$.

5.13 Mean velocity: Sensitivity of prediction to changes log-law of the wall constant, $C$. Prediction of analytical solution and experimental data of Tsuji et al.[44], $R e \approx 23000$

5.14 Turbulence intensity: Sensitivity of prediction to changes in log-law of the wall constant, $C$. Prediction of experimental data of Tsuji et al.[44]. $R e \approx 23000 \ldots \ldots \ldots . \ldots . \ldots . \ldots . . \ldots 6$

5.15 Particle path in a wedge-shape geometry ............ 6.5

5.16 Circular Cross-Section . . . . . . . . . . . . . . 66

5.17 Particle Dispersion: Prediction of Vames and Hanratty's [46] experimental results using $\overline{x^{2}}=\overline{r^{2}} / 2, \ldots \ldots \ldots . \ldots \ldots 9$

5.18 Analytical solution and code predictions for $\epsilon \ldots \ldots . \ldots$

5.19 Analytical solution and code predictions for $k \ldots \ldots \ldots$

6.1 Experimental setup ......................... 76

6.2 Mean velocity: Predictions of experimental data of Tsuji et al[44], $R e \approx$

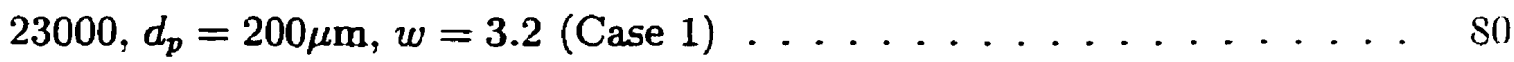

6.3 Turbulence intensity: Predictions of experimental data of Tsuji et al[44]. $R e \approx 23000, d_{p}=200 \mu \mathrm{m}, w=3.2($ Case 1) . . . . . . . . . . .

6.4 Effect of doubling number of particles injected: Turbulence intensity predictions of experimental data of Tsuji et al[44], $R e \approx 23000, d_{p}=200 \mu \mathrm{m}$. $w=3.2($ Case 1$) \ldots \ldots \ldots$

6.5 Turbulent Viscosity, $\mu_{t}$ : Predictions from the models and clean solution. Case 1. .......................... 81

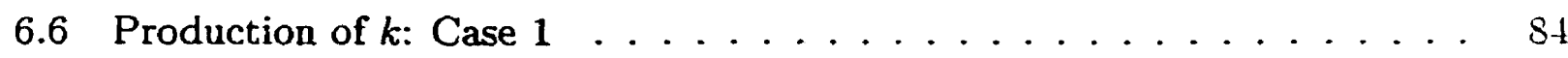

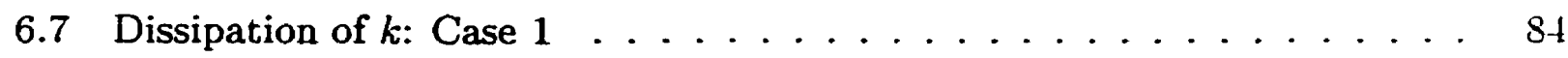

6.8 Source to $k$ equation, $S_{k}$ : Case $1 \ldots \ldots \ldots$.j

6.9 Total source to $k$ equation, Production $-\epsilon-S_{k}$ : Case $1 \ldots \ldots$.j

6.10 Mean velocity: Predictions of experimental data of Tsuji et al[44]. $R e \approx$ $23000, d_{p}=200 \mu \mathrm{m}, w=1.3$ (Case 2) . . . . . . . . . . si

6.11 Turbulence intensity: Predictions of experimental data of Tsuji et al[4t].

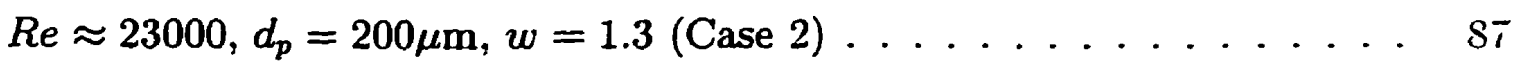

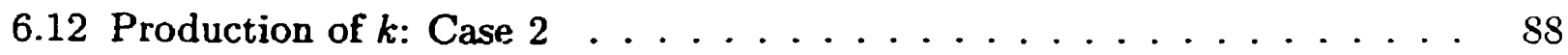


6.13 Dissipation of $k$ : Case $2 \ldots \ldots \ldots \ldots$. . . . . . . . . . . .

6.14 Source to $k$ equation, $S_{k}$ : Case $2 \ldots \ldots \ldots \ldots$

6.15 Total source to $k$ equation, Production $-\epsilon-S_{k}$ : Case $2 \ldots \ldots$

6.16 Turbulent Viscosity, $\mu_{t}$ : Predictions from the models and clean solution. Case 2. . . . . . . . . . . . . . . . . . 90

6.17 Mean velocity: Predictions of experimental data of Tsuji et al[44], Re $\approx$ $22000, d_{p}=500 \mu \mathrm{m}, w=3.4$ (Case 3$) \ldots \ldots . . \ldots 91$

6.18 Turbulence intensity: Predictions of experimental data of Tsuji et al[44]. $R e \approx 22000, d_{p}=500 \mu \mathrm{m}, w=1.3($ Case 3$) \ldots \ldots$ 91

6.19 Production of $k$ : Case $3 \ldots \ldots \ldots 2$

6.20 Dissipation of $k$ : Case $3 \ldots \ldots \ldots \ldots . \ldots \ldots$

6.21 Source to $k$ equation, $S_{k}$ : Case $3 \ldots \ldots . \ldots . \ldots . \ldots$

6.22 Total source to $k$ equation, Production $-\epsilon-S_{k}$ : Case $2 \ldots . . \ldots 9$

6.23 Turbulent Viscosity, $\mu_{t}$ : Predictions from the models and clean solution. Case 2. . . . . . . . . . . . . . . . . . 94

6.24 Chen and Wood: Prediction of change in turbulence intensity . . . . . 9j

$6.25 \mathrm{Tu}$ and Fletcher: Prediction of change in turbulence intensity . . . . . 95

6.26 Mostafa and Mongia: Prediction of change in turbulence intensity . . . 9 96

6.27 Lightstone and Hodgson: Prediction of change in turbulence intensity . . 96

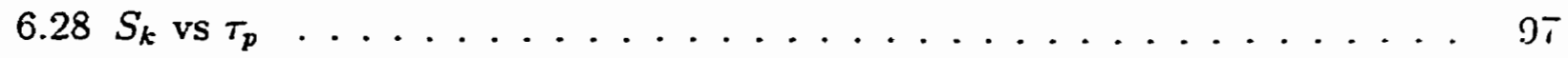




\title{
Nomenclature
}

\author{
SYMBol Definition \\ English \\ $A$ \\ $B_{k}, B_{\epsilon}$ \\ $C$ \\ $C(r)$ \\ $C_{D}$ \\ $C_{\epsilon 3}$ \\ $C_{\mu}, C_{1}, C_{2}$ \\ $d_{p}$ \\ $D$ \\ $D_{p}$ \\ $f$ \\ $f_{r}(r)$ \\ $f_{x}(x)$ \\ $F_{x y}(x, y)$ \\ $F$ \\ $F_{e}$ \\ $F_{p}$ \\ $F_{p}^{\prime}$ \\ $\frac{p}{F_{p}}$ \\ $g$ \\ $i, j, k$ \\ $k$ \\ $l_{e}$ \\ $l_{w}$ \\ $L_{\epsilon}$ \\ $L_{h}$

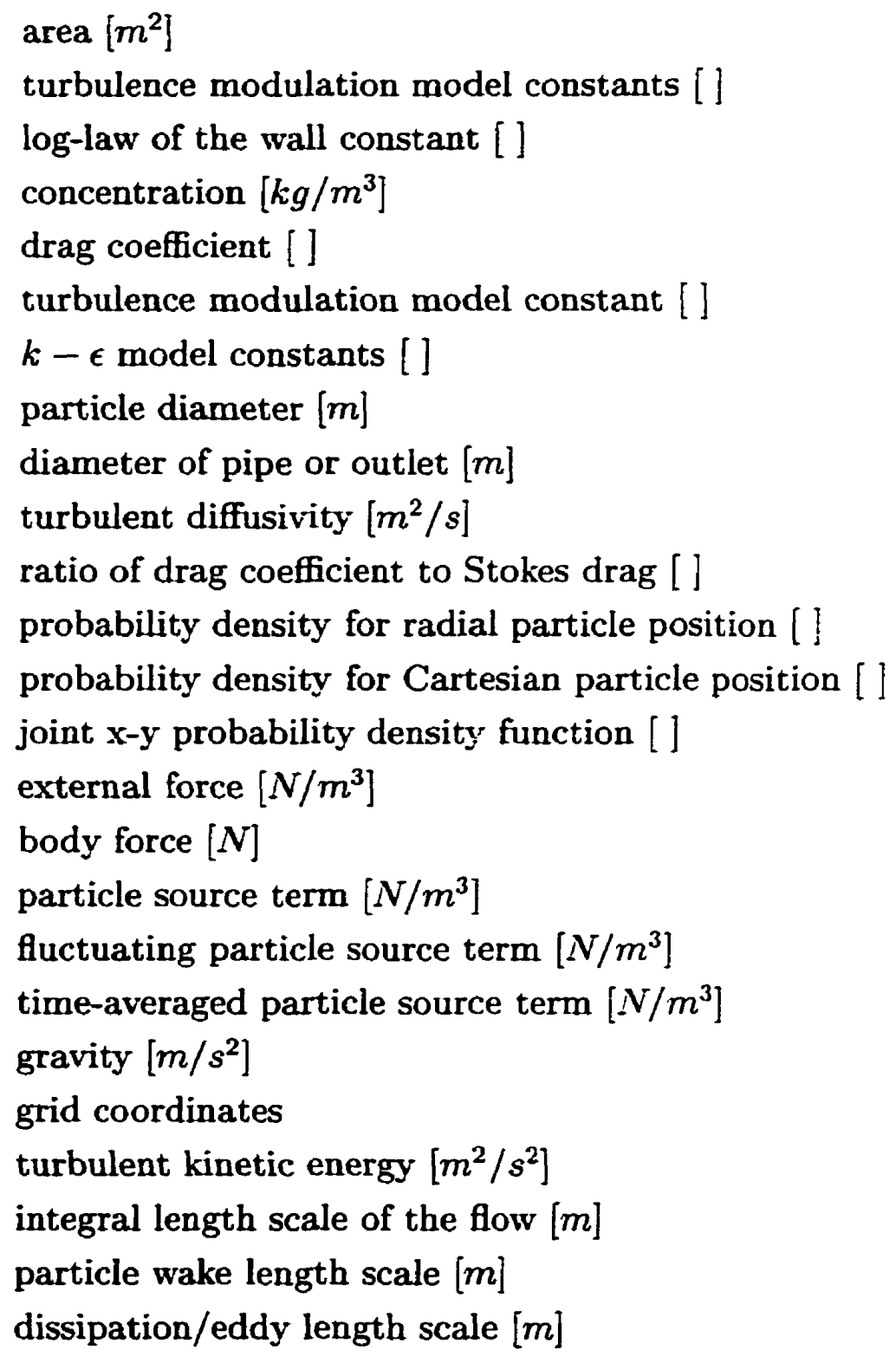




\begin{tabular}{|c|c|}
\hline SYMBOL & DEFINITION \\
\hline$L_{I}$ & Lagrangian integral length scale $[m]$ \\
\hline$\dot{m}$ & mass flow rate $[\mathrm{kg} / \mathrm{s}]$ \\
\hline$m_{p}$ & particle mass $[\mathrm{kg}]$ \\
\hline$n_{j}$ & outward normal surface vector \\
\hline$N_{n}$ & shape functions [] \\
\hline$P$ & pressure $\left[n / m^{2}\right]$ \\
\hline$P_{d}$ & energy production by fluid in presence of particles $\left[\mathrm{m}^{2} / \mathrm{s}^{3}\right]$ \\
\hline$P_{i}$ & inherent turbulence production $\left[\mathrm{m}^{2} / \mathrm{s}^{3}\right]$ \\
\hline$P_{\mathrm{\epsilon}}$ & rate of energy dissipation $[\mathrm{Nm} / \mathrm{s}]$ \\
\hline$r, \theta, z$ & cylindrical coordinates \\
\hline$R$ & pipe radius $[m]$ \\
\hline$R e$ & Reynolds number [] \\
\hline$R e_{p}$ & particle Reynolds number [] \\
\hline$R_{g p}$ & autocorrelation function [] \\
\hline$R_{E U L}$ & Eulerian spatial correlation [] \\
\hline$R_{L A G}$ & Lagrangian temporal correlation [] \\
\hline $\operatorname{Re}$ & Reynolds number [] \\
\hline$R e_{p}$ & particle Reynolds number [] \\
\hline$S_{\epsilon}$ & source to $\epsilon$ equation $\left[\mathrm{m}^{2} / \mathrm{s}^{4}\right]$ \\
\hline$S_{k}$ & source to $k$ equation $\left[\mathrm{m}^{2} / \mathrm{s}^{3}\right]$ \\
\hline$t$ & time $[s]$ \\
\hline$T_{i}$ & turbulence intensity [ ] \\
\hline$u$ & instantaneous velocity $[\mathrm{m} / \mathrm{s}]$ \\
\hline$u^{\prime}$ & fluctuating velocity $[\mathrm{m} / \mathrm{s}]$ \\
\hline$U, V, W$ & time-averaged velocity $[\mathrm{m} / \mathrm{s}]$ \\
\hline$U_{c}$ & mean centreline velocity $[\mathrm{m} / \mathrm{s}]$ \\
\hline$u_{R E L}$ & relative velocity between the phases $[\mathrm{m} / \mathrm{s}]$ \\
\hline$u_{\tau}$ & velocity tangent to the wall $[\mathrm{m} / \mathrm{s}]$ \\
\hline$u^{+}$ & dimensionless log-law of the wall velocity [ ] \\
\hline$\forall$ & volume $\left[m^{3}\right]$ \\
\hline$w$ & loading ratio [] \\
\hline$W_{e}$ & work done by eddy on a particle $[\mathrm{Nm}]$ \\
\hline$x, y, z$ & Cartesian coordinates \\
\hline$y^{+}$ & dimensionless log-law of the wall length [ ] \\
\hline
\end{tabular}


SYMBOL

\section{Greek}

$\alpha$

$\Gamma$

$\delta_{i j}$

$\delta t$

$\Delta t$

$\Delta n$

$\epsilon$

$\lambda$

$\mu$

$\mu_{\text {eff }}$

$\mu_{t}$

$\nu$

$\phi$

$\rho$

$\rho_{s}$

$\sigma^{2}$

$\sigma_{k}, \sigma_{\epsilon}$

$\tau_{c}$

$\tau_{e}$

$\tau_{\text {int }}$

$\tau_{L I}$

$\tau_{p}$

$\tau_{t r}$

$\tau_{w}$

$\tau^{*}$

Subscripts

$g$

$p$

Abbreviations

CDS

LP

MW
Definition

particle concentration $\left[\mathrm{kg} / \mathrm{m}^{3}\right]$

normally distributed random variable

Kronecker delta []

timestep $[s]$

timestep $[s]$

distance form wall to near-wall node $[\mathrm{m}]$

kinetic energy dissipation rate $\left[\mathrm{m}^{2} / \mathrm{s}^{3}\right]$

average interparticle spacing $[\mathrm{m}]$

viscosity $\left[\mathrm{Ns} / \mathrm{m}^{2}\right]$

effective viscosity $\left[\mathrm{Ns} / \mathrm{m}^{2}\right]$

turbulent viscosity $\left[\mathrm{Ns} / \mathrm{m}^{2}\right]$

kinematic viscosity $\left[\mathrm{m}^{2} / \mathrm{s}\right]$

particulate phase volume fraction []

density $\left[\mathrm{kg} / \mathrm{m}^{3}\right]$

particulate phase loading []

variance

$k-\epsilon$ model constants []

time between particle collisions $[s]$

eddy lifetime $[s]$

interaction time $[s]$

Lagrangian integral timescale $[s]$

particle response time $[s]$

particle transit time $[s]$

wall shear stress $\left[N / \mathrm{m}^{2}\right]$

integral timescale of fluid velocity along particle path $[s]$

gas-phase

particle-phase

Central Difference Scheme

Linear Profile

Mass Weighted 
SYMBOL

PAC

PDF

SUD

SSF
Definition

Physical Advection Correction

Probability Density Function

Skewed Upstream Difference

Stochastic Separated Flow 


\section{Chapter 1}

\section{Introduction}

\subsection{Motivation}

Two-phase turbulent flows occur in a variety of industrial and natural processes. Thesc processes include the combustion of liquid and solid fuels, pneumatic transport of particulate materials, exhaust plumes and environmental particulate transport.

For sufficiently dense two-phase flows, turbulence modulation will occur. Turbulence modulation refers to the process whereby the fluid phase turbulence is modified by the presence of the particles. This modification can cause an increase or a decrease in the mean velocity and turbulence intensity of the flow.

There have been significant advancements in the past two decades in the ability of turbulence models to produce good predictions of the fluid phase. This has led to the development of increasingly accurate particle dispersion models. Building on this. the next step is the development of a model which can capture the change in the flow field due to the particles.

This thesis is concerned with the understanding and modeling of turbulence modillation for gas-particle pipe flow. The underlying turbulence model is the two-equation $k-\epsilon$ turbulence closure model and a Stochastic Separated Flow model is used to model the particle dispersion. The flow is sufficiently dilute that particle-particle interactions can be neglected.

\subsection{Objectives and Scope}

The objectives of this thesis are outlined below:

1. To understand the processes involved in turbulence modulation. 
2. To investigate the ability of existing turbulence modulation models to predict experimental gas-particle turbulent pipe flow results.

3. To develop a model to predict turbulence modulation.

This investigation involves incorporating the new and existing turbulence modulation models into a commercial computational fluid dynamics code, TASCflow. The underlying turbulence model is the two-equation $k-\epsilon$ turbulence closure model. Thus. the focus of this work is those turbulence modulation models which can be implemented into the $k-\epsilon$ framework.

Effective modeling of turbulence modulation inherently requires a good particle dispersion model. For this investigation a Stochastic Separated Flow model, which solves for the individual particle trajectories, will be used to determine particle concentrations.

\subsection{Outline of Thesis}

Chapter 2 provides background information and an introduction to turbulence modulation. Key parameters affecting turbulence modulation are discussed and experimental trends examined. The governing equations for the gas and particle-phases are presented and the turbulent kinetic energy and dissipation equations derived. The $k-\epsilon$ model is presented as is the SSF model used in this investigation. Finally; existing turbulence modulation models are presented and discussed.

Chapter 3 presents the new model of Lightstone and Hodgson. Motivation for the model is discussed and the model is derived in full.

Chapter 4 outlines the numerical methods used to solve the governing equations. Discretization and evaluation of the equations is discussed and relevant boundary condition treatment presented. The numerical solution of the particulate-phase is presented in detail, including the implementation of the SSF model and source terms. Finally: the' procedure developed in this work for implementing the turbulence modulation models is introduced.

Chapter 5 describes the validation performed in this research. The validations began with a laminar pipe flow solution checked against the analytical solution. I turbulent pipe flow simulation was then performed and the results compared to both experimental data and the analytical solution. Sensitivity of this solution to changes in inlet conditions. node distribution and the log-law of the wall constant is examined and grid-independence established. The ability of the CFD code to predict the particle dispersion is rerified bycomparing the solution from the simulation to experimental data. An analytical solution 
to the $k$ and $\epsilon$ equations including a source term was derived for a grid-turbulent flow: and the ability of the code to implement the source terms established. Finally it is shown analytically that the code properly implements source terms to the momentum equations.

Chapter 6 presents the results of this investigation. The turbulence modulation models investigated are: Chen and Wood [4], Tu and Fletcher [45], Mostafa and Mongia [30] and the model of Lightstone and Hodgson. The experiment of Tsuji et al [44] is used to evaluate these turbulence modulation models. The models are run in three different cases in order to determine the models' effectiveness at capturing experimental and general trends. Analytical work is performed to determine the behaviour of the models in flows with varying particle size and density.

Chapter 7 provides a summary of the thesis, conclusions and suggestions for future work are presented. 


\section{Chapter 2}

\section{Background}

This chapter presents the underlying theory behind the phenomenon of turbulence modulation. A comprehensive review of experimental work that focuses on turbulence modulation is given and conclusions are drawn as to which parameters and mechanisms produce turbulence modulation. The governing equations for the gas-phase are outlined including the derivation of the $k$ and $\epsilon$ equations, the cornerstones of the models used in this research.

A review of the existing models are presented in the latter portion of the chapter. A stochastic separated flow particle dispersion model and the $k$ and $\epsilon$ turbulence model form the basis for this research. It is through the $k-\epsilon$ model that turbulence modulation effects are implemented. Finally, a review of existing turbulence modulation models is given, including both those models which can be incorporated into the $k-\epsilon$ model and theoretical models which provide further insight into the modeling of turbulence modulation.

\subsection{Experimental Trends}

The presence of particles in a turbulent flow may result in an increase or decrease of the turbulent kinetic energy and mean velocity of the gas-phase. This phenomenon of turbulence modulation has been investigated experimentally by several researchers. Tsuji and Morikawa (1982)[43], Tsuji et al. (1984)[44], Lee and Durst (1982)[22] and Fan et al (1997) [12] looked at turbulence modulation in solid-gas pipe flow while Mostafa and Mongia (1989)[31], Levy and Lockwood (1981)[23], Modarress et al (1984)[28] and Modarress et al (1984)[29] looked turbulence modulation in solid-gas jet flow. Results from these studies are presented in Table 2.1. The general experimental trends are 
discussed below.

\subsubsection{Parameters Affecting Turbulence Modulation}

There are two primary parameters which govern turbulence modulation: the particle diameter and the mass loading ratio of the flow. These parameters are discussed in turn below.

Experimental data shows that the magnitude and type (increase or decrease) of turbulence modulation is highly dependent on particle size. Three particle diameter regimes can be identified. The first regime consists small particles with diameter less than $\sim 250 \mu \mathrm{m}$ (but greater than the very small particles that can exactly follow the fluicl flow; these particles will not affect the flow field). These particles tend to cause a reduction in gas-phase turbulence. At the other end of the scale, particles with diameters greater than $\sim 800 \mu \mathrm{m}$ tend to increase the gas-phase turbulence. The intermediate regim contains particles with diameters greater than $\sim 250 \mu \mathrm{m}$ and less than $\sim 800 \mu \mathrm{m}$. These particles can serve to either increase or decrease the gas-phase turbulence [23].

It is more useful to express the particle diameter in terms of a dimensionless parameter. In their 1989 study, Gore and Crowe [14] examined the effects of particle size on turbulence modulation. The study examined the data from fifteen experiments dealing with turbulence modulation. The data were reduced and the percent change in turbulence intensity was plotted as a function of length scale ratio, $\frac{d_{p}}{l_{e}}$, where $d_{p}$ is the particle diameter and $l_{e}$ is the integral length scale of the flow. The study found that for $\frac{d_{p}}{l_{\text {. }}}$ greater than 0.1 the particles increased the percent change in turbulence intensity while for $\frac{d_{p}}{l_{e}}$ less than 0.1 the percent change in turbulence intensity was decreased.

The loading ratio of the flow, $w$, is defined as the ratio of the total mass flow rate of the particles to the total mass flow rate of the gas-phase,

$$
w=\frac{\dot{m_{p}}}{\dot{m}_{g}}
$$

The loading ratio will influence the density, or conversely, the diluteness of the flow. A dilute flow is a flow in which the particle motion is controlled by surface and body. forces acting on the particle (for example fluid drag and gravity). This results in one or two-way coupling between the particles and the fluid. In a dilute flow. particleparticle communication is through the gas-phase. A dense flow is a flow in which the particle motion is controlled by interparticle collisions. In addition to two-way coupling between the particles and the fluid, there is also coupling within the particulate-phase 
(the particle-particle interactions) and it is through these interparticle collisions that communication between particles is direct. This type of interaction is called four-way coupling [7]. A quantitative parameter distinguishing dilute flows from dense flors is the ratio of the particle response time, $\tau_{p}$, to the time between particle collisions. $\tau_{c}$. The particle response time, $\tau_{p}$, is defined as the time required for a particle release from rest to accelerate to $63 \%$ of the free stream velocity. If $\tau_{p} / \tau_{c}<1$ the flow is dilute since the particle has time to respond to the gas velocity field before the next collision. If. however, $\tau_{p} / \tau_{c}>1$, the particle motion is controlled by collisions and the flor is clense [6]. This work will focus only on dilute flows with loading ratios high enough to exhibit two-way coupling.

Turbulence modulation occurs in flows with loading ratios greater than 0.1 [30]. Experimental results show that the effect of increasing the loading ratio differs depending on particle size. For large particles, an increase in the loading ratio serves to further increase the turbulent kinetic energy of the flow. For small particles, the effect is mixed. As the loading ratio is initially increased there is a further reduction in turbulent kinetic energy: As the loading ratio is increased even more the magnitude of the turbulence reduction begins to decrease. An explanation for this trend is offered later in this section.

Elghobashi (1994) [11] proposed a relationship between the related parameter of volume fraction and particle-turbulence interaction. He found that for volume fractions less than $10^{-6}$ the particles will not influence the flow. For volume fraction between $10^{-6}$ and $10^{-3}$ two-way coupling will occur in the flow. Flows with volume fractions above $10^{-3}$ will exhibit particle-particle interactions as well as two-way coupling.

\subsubsection{Mechanisms of Modulation}

There are four mechanisms through which turbulence modulation can occur:

(1) Energy transfer through the drag force

(2) Increased energy dissipation through particle collisions with turbulent eddies

(3) Energy production by the particles through vortex shedding and wake effects

(4) Crossing trajectory effect

The first two mechanisms will generally serve to reduce the turbulent kinetic energy of the flow. The third and fourth will increase it.

\section{Drag Force}

The gas-phase turbulence can be modified as a result of energy transfer to the particle through the drag force. In most cases this interaction acts as a sink of turbulent kinetic 
energy. If, however, the particle velocity is greater than the fluid velocity (due to initial conditions, for example) this interaction can act as a source of turbulent kinetic energyto the gas-phase. Reduction of the gas-phase turbulent kinetic energy as a result of the drag force is predominant in particles in small to intermediate size ranges $(<\sim 800, \mathrm{~mm})$. This is the mechanism that is the focus of most turbulence modulation models.

\section{Increased Dissipation}

The turbulent kinetic energy of the gas-phase can also be reduced through particle collisions with turbulent eddies. A particle may collide with an eddy of a comparable length scale, causing the eddy to break up into smaller eddies. If these smaller eddies are on the same length scale as the eddies responsible for the dissipation of turbulent kinetic. energy then the energy dissipation in the flow will increase. This results in a decrease in the turbulence of the flow.

\section{Wake Formation and Vortex Shedding}

Particles moving through the gas-phase will create disturbances in the flow field by wake formation and vortex shedding. This is the primary mechanism through which the turbulent kinetic energy of the gas-phase is increased. Experiments have shown that for at particle Reynolds number, $R e_{p}$, greater than twenty,

$$
R e_{p}=\frac{\left|\overrightarrow{u_{g}}-\overrightarrow{u_{p}}\right| d_{p}}{\nu}>20
$$

wake formation will occur. Flows with $R e_{p}>400$ will exhibit vortex shedding which will cause an even greater increase in the turbulent kinetic energy of the gas-phase through the production of turbulence [47]. Larger particles (> $800 \mu \mathrm{m})$ moving through the gas will produce wakes of a length scale near the integral length scale of the flow. These wakes will increase the turbulence intensity of the gas-phase, transferring energy from the mean flow to the turbulent kinetic energy.

Smaller particles can also exhibit wake formation and vortex shedding. At low loarling ratios these wakes will not significantly affect the flow field. As the loading ratio increases, these small-scale wakes may interact with each other and the amplified effect may cause a significant increase in the turbulent kinetic energy of the flow field. This is one explanation for the observed experimental trend for smaller particles, where the turbulence is initially decreased but as the loading ratio is increased the turbulence intensity also increases. 


\section{Crossing Trajectory Effect}

The crossing trajectory effect may also cause an increase in the turbulent kinetic energ: of the gas-phase. The crossing trajectory effect is caused by a large relative mean relocity. between the particles and the turbulent eddies. The particles will fall through the eddies dragging the mean gas flow along. This results in an increase in the mean relociry gradients and consequently the turbulent kinetic energy of the flow field [23].

\subsubsection{Mean Gas-Phase}

The effects of particle diameter and loading ratio on the mean velocity of the gas-phase are not as clear. An increase or a decrease of the mean gas-phase velocity can occur for any particle size; there is no evident way to categorize particle diameters in terms of an increase or a decrease of the mean velocity. Varying the loading ratio has the same effect on the mean momentum as it did on the turbulent kinetic energy.

In two-phase flows the mean velocity of the gas-phase is affected by both the Reynolds: stresses of the gas-phase and the relative velocity between the gas and particulate-phases. Therefore the modification of the mean gas-phase velocity will depend on both the turbulent flow field of the gas-phase and parameters associated with the particulate-phase. It is not clear how to uncouple these effects in order to isolate the effect of the particulatephase on the mean velocity.

\subsection{Physics of the Flow}

\subsubsection{Particle Equation of Motion}

The equation of motion for a discrete particle in a fluid is based on Newton's second law: the product of the mass and acceleration of a particle is equal to the forces acting on the particle. The equation of motion for a spherical particle, derived by Basset. Boussinesc 1 and Oseen [18] is as follows:

$$
\begin{aligned}
\underbrace{m_{p} \frac{d \overrightarrow{u_{p}}}{d t}}_{I}= & \underbrace{3 \pi \mu d_{p}\left(\overrightarrow{u_{g}}-\overrightarrow{u_{p}}\right)}_{I I}+\underbrace{\frac{\pi}{6} d_{p}^{3} \rho_{g} \frac{d \overrightarrow{u_{g}}}{d t}}_{I I I}+\underbrace{\frac{1}{2} \frac{\pi}{6} d_{p}^{3} \rho_{g}\left(\frac{d \overrightarrow{u_{g}}}{d t}-\frac{d \overrightarrow{u_{p}}}{d t}\right)}_{I V} \\
& +\underbrace{\frac{3}{2} d_{p}^{2} \sqrt{\pi \rho_{g} \mu} \int_{t}^{t_{0}} \frac{\frac{d}{d t}\left(\overrightarrow{u_{g}}-\overrightarrow{u_{p}}\right)}{\sqrt{t-t^{\prime}}} d t^{\prime}}_{V}+\underbrace{F_{e}}_{V I}
\end{aligned}
$$




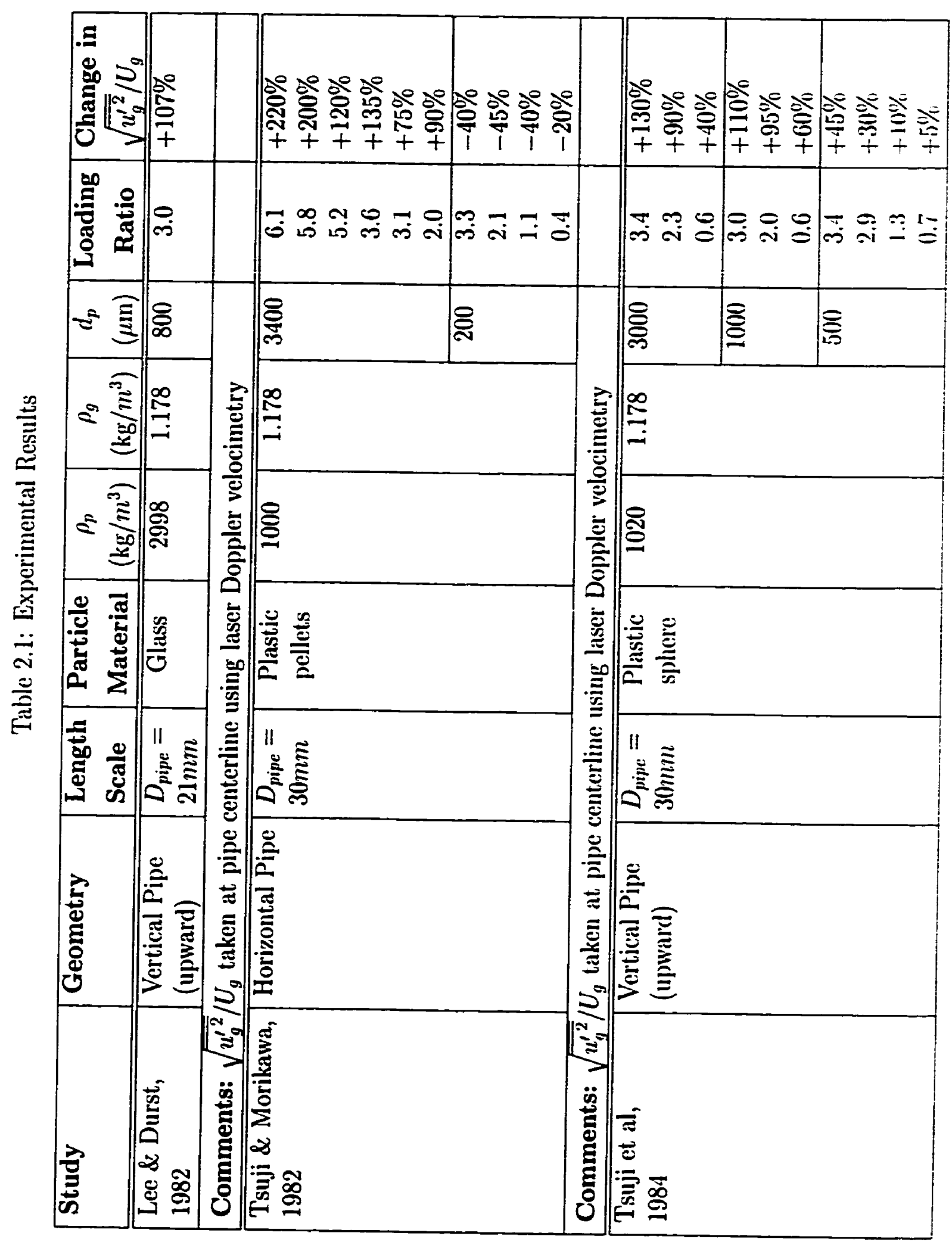


Table 2.1 (Continued)

\begin{tabular}{|c|c|c|c|c|c|c|c|c|}
\hline Study & Geometry & $\begin{array}{l}\text { Length } \\
\text { Scale }\end{array}$ & $\begin{array}{c}\text { Particle } \\
\text { Material }\end{array}$ & $\begin{array}{c}\rho_{p} \\
\left(\mathrm{~kg} / m^{3}\right)\end{array}$ & $\begin{array}{c}\rho_{g} \\
\left(\mathrm{~kg} / \mathrm{m}^{3}\right)\end{array}$ & $\begin{array}{c}d_{p} \\
(\mu \mathrm{m})\end{array}$ & $\begin{array}{c}\text { Loading } \\
\text { Ratio }\end{array}$ & $\begin{array}{l}\text { Change in } \\
\sqrt{\overline{u_{g}^{\prime 2}}} / U_{g}\end{array}$ \\
\hline $\begin{array}{l}\text { Tsuji et al, } \\
1984 \\
\text { (continued) }\end{array}$ & & & & & & 200 & $\begin{array}{l}3.2 \\
1.9 \\
1.3 \\
0.9 \\
0.5\end{array}$ & $\begin{array}{l}-5 \% \\
-20 \% \\
-30 \% \\
-10 \% \\
-10 \%\end{array}$ \\
\hline \multicolumn{9}{|c|}{ Comments: $\sqrt{u_{g}^{\prime 2}} / U_{g}$ taken at pipe centerline using laser Doppler velocimetry } \\
\hline $\begin{array}{l}\text { Fan et al, } \\
1997\end{array}$ & $\begin{array}{l}\text { Vertical Pipe } \\
\text { (upward) }\end{array}$ & $\begin{array}{l}D_{p i p e}= \\
100 m m\end{array}$ & Quartz & 2200 & 1.178 & 100 & $\begin{array}{l}2.0 \\
1.0\end{array}$ & $\begin{array}{l}-24 \% \\
-29 \%\end{array}$ \\
\hline \multicolumn{9}{|c|}{ Comments: $\sqrt{u_{g}^{\prime 2}} / U_{g}$ taken at pipe centerline using laser Doppler velocimetry } \\
\hline \multirow{5}{*}{$\begin{array}{l}\text { Levy \& Lockwood, } \\
1981\end{array}$} & \multirow{5}{*}{$\begin{array}{l}\text { Vertical Jet } \\
\text { (downward) }\end{array}$} & \multirow{5}{*}{$\begin{array}{l}D_{\text {outlet }}= \\
15 \mathrm{~mm}\end{array}$} & \multirow[t]{5}{*}{ Sand } & \multirow[t]{5}{*}{2620} & \multirow[t]{5}{*}{1.178} & 1050 & 3.5 & $+2 \%$ \\
\hline & & & & & & 750 & 2.4 & $-2 \%$ \\
\hline & & & & & & 540 & 2.2 & $-3 \%$ \\
\hline & & & & & & 400 & 1.2 & $-3 \%$ \\
\hline & & & & & & 215 & $\begin{array}{l}1.1 \\
2.3\end{array}$ & $\begin{array}{l}-2 \% \\
-16 \% \\
\end{array}$ \\
\hline \multicolumn{9}{|c|}{ Comments: $\sqrt{u_{g}^{\prime 2}} / U_{g}$ taken at jet centerline, $\mathrm{x} / \mathrm{D}=20$ using laser Doppler velocimetry } \\
\hline \multirow{3}{*}{$\begin{array}{l}\text { Shuen et al, } \\
1983\end{array}$} & \multirow{3}{*}{$\begin{array}{l}\text { Vertical Jet } \\
\text { (downward) }\end{array}$} & \multirow{3}{*}{$\begin{array}{l}D_{\text {outlet }}= \\
10.9 \mathrm{~mm}\end{array}$} & \multirow[t]{3}{*}{ Sand } & \multirow[t]{3}{*}{2620} & \multirow[t]{3}{*}{1.178} & 207 & 0.66 & $+11 \%$ \\
\hline & & & & & & 119 & $\begin{array}{c}0.66 \\
0.2\end{array}$ & $\begin{array}{l}+11 \% \\
+15 \%\end{array}$ \\
\hline & & & & & & 79 & 0.20 & $+17 \%$ \\
\hline \multicolumn{9}{|c|}{ Comments: $\sqrt{u_{g}^{\prime 2}} / U_{g}$ taken at jet, centerline, $x / D=20$} \\
\hline $\begin{array}{l}\text { Modarress ot al, } \\
1981\end{array}$ & $\begin{array}{l}\text { Vertideal Jet } \\
\text { (downward) }\end{array}$ & $\begin{array}{l}D_{\text {oullil }}= \\
2()_{1 \mathrm{~m}, \mathrm{~m}}\end{array}$ & Cilasis & 2990 & 1.178 & 50 & $\begin{array}{l}0.85 \\
0.32\end{array}$ & $\begin{array}{l}-19 \% \\
-13 \%\end{array}$ \\
\hline \multicolumn{9}{|c|}{ 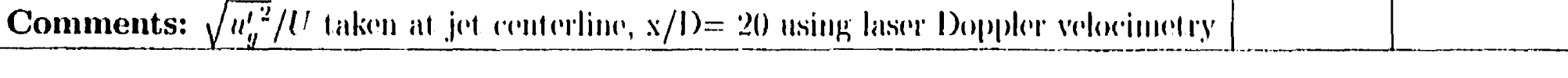 } \\
\hline
\end{tabular}


Table 2.1 (Continued)

\begin{tabular}{|c|c|c|c|c|c|c|c|c|}
\hline Study & Geometry & $\begin{array}{l}\text { Length } \\
\text { Scale }\end{array}$ & $\begin{array}{c}\text { Particle } \\
\text { Material }\end{array}$ & $\begin{array}{c}\rho_{p} \\
\left(\mathrm{~kg} / m^{3}\right) \\
\end{array}$ & $\begin{array}{c}\rho_{g} \\
\left(\mathrm{~kg} / \mathrm{m}^{3}\right) \\
\end{array}$ & $\begin{array}{c}d_{p} \\
(\mu \mathrm{mm})\end{array}$ & $\begin{array}{c}\text { Loading } \\
\text { Ratio }\end{array}$ & $\begin{array}{l}\text { Change in } \\
\sqrt{u_{g}^{\prime{ }^{\prime 2}}} / U_{g}\end{array}$ \\
\hline $\begin{array}{l}\text { Modarress, Wuerer, } \\
\text { \& Elghobashi, } 1984\end{array}$ & $\begin{array}{l}\text { Vertical Jet } \\
\text { (downward) }\end{array}$ & $\begin{array}{l}D_{\text {outlet }}= \\
20 \mathrm{~mm}\end{array}$ & 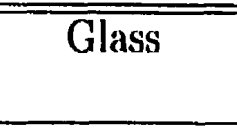 & 2990 & 1.178 & 200 & 0.8 & $-13 \%$ \\
\hline \multicolumn{9}{|c|}{ Comments: $\sqrt{u_{g}^{\prime 2}} / U_{g}$ taken at jet centerline, $\mathrm{x} / \mathrm{D}=20$ using laser Doppler velocimetry } \\
\hline \multirow{3}{*}{$\begin{array}{l}\text { Shuen et al, } \\
1985\end{array}$} & \multirow{3}{*}{$\begin{array}{l}\text { Vertical Jet } \\
\text { (downward) }\end{array}$} & \multirow{3}{*}{$\begin{array}{l}D_{\text {outlet }}= \\
10.9 \mathrm{~mm}\end{array}$} & \multirow[t]{3}{*}{ Sand } & \multirow[t]{3}{*}{$\overline{\overline{2620}}$} & \multirow[t]{3}{*}{1.178} & $\overline{207}$ & 0.66 & $-3 \%$ \\
\hline & & & & & & 119 & $\begin{array}{l}0.66 \\
0.20\end{array}$ & $\begin{array}{l}-20 \% \\
-8 \%\end{array}$ \\
\hline & & & & & & 79 & 0.20 & $-5 \%$ \\
\hline \multicolumn{9}{|c|}{ Comments: $k /{\overline{U_{g c}}}^{2}$ taken at jet centerline, $\mathrm{x} / \mathrm{D}=20$} \\
\hline $\begin{array}{l}\text { Mostafa et al, } \\
1989\end{array}$ & $\begin{array}{l}\begin{array}{l}\text { Axisymmetric Jet } \\
\text { (downward) }\end{array} \\
\end{array}$ & $\begin{array}{l}D_{\text {outlet }}= \\
25 \mathrm{~mm}\end{array}$ & Glass Beads & 2500 & 1.178 & 105 & $\begin{array}{l}1.0 \\
0.2\end{array}$ & $\begin{array}{l}-16 \% \\
-23 \% \\
\end{array}$ \\
\hline
\end{tabular}


Term (I) is the force required to accelerate the particle. Term (II) represents the hydrodynamic drag force, based on the relative velocity of the particle and the gas. assuming Stokes flow. Term (III) is the force due to the pressure gradient in the gas surrounding the particle, caused by the acceleration of the gas. Term (IV) is the "added mass" force; the additional force required to accelerate the virtual mass of the thuid. Term (V) is the Basset History force which accounts for the effect of the deviation of the flow pattern from steady state. Finally, Term (VT) represents the body force term which may include gravity.

Dividing Eq. (2.3) by the particle mass and rearranging yields the following form of the particle equation of motion:

$$
\begin{aligned}
\frac{d \vec{u}_{p}}{d t}= & \frac{1}{\tau_{p}}\left(\vec{u}_{g}-\vec{u}_{p}\right)+\frac{\rho_{g}}{\rho_{p}} \frac{d \vec{u}_{g}}{d t}+\frac{1}{2} \frac{\rho_{g}}{\rho_{p}}\left(\frac{d \vec{u}_{g}}{d t}-\frac{d \vec{u}_{p}}{d t}\right) \\
& +\frac{3}{\sqrt{2 \pi \tau_{p}}} \sqrt{\frac{\rho_{g}}{\rho_{p}}} \int_{t}^{t_{o}} \frac{\frac{d}{d t}\left(\overrightarrow{u_{g}}-\overrightarrow{u_{p}}\right)}{\sqrt{t-t^{\prime}}} d t^{\prime}+\frac{6}{\rho_{p} \pi d^{3}} F_{e}
\end{aligned}
$$

where $\tau_{p}$ is the particle, or aerodynamic, response time of the particle given by:

$$
\tau_{p}=\frac{d^{2} \rho_{p}}{18 \mu}
$$

The particle response time is a reflection of the inertia and size of the particle. It is typically defined as the time required for a particle at rest to be accelerated within approximately $63 \%$ of the fluid velocity [17]. Small, light particles, corresponding to small values $\tau_{p}$, will follow the high frequency fluctuations of the turbulence while larger. heavy particles will have a reduced response to the turbulence.

For the gas-solid flows considered, the ratio of the gas density to the particle density is small, on the order of $10^{-3}$. Therefore, the only terms on the right hand side of Eq. (2.t) that are important are the drag force and the body force. The only body force acting on the particle for the flow considered is gravity, so the particle equation of motion reduces to:

$$
\frac{d \vec{u}_{p}}{d t}=\frac{1}{\tau_{p}}\left(\vec{u}_{g}-\vec{u}_{p}\right)+\vec{g}
$$

To account for the effect of the drag force for flows beyond Stokes regime $\left(R e_{p}<<1\right)$ a correction factor should be included in the drag term. The correction factor. $f$. is obtained from the standard drag curve based on a laminar free-stream and is expressed 
as:

$$
f=\left(1+0.15 \operatorname{Re}_{p}^{0.687}\right) \quad \operatorname{Re}_{p}<700
$$

where $R e_{p}$ is given by:

$$
R e_{p}=\frac{\left|\vec{u}_{g}-\vec{u}_{p}\right| d_{p}}{\nu}
$$

Therefore, the particle equation of motion becomes:

$$
\frac{d \vec{u}_{p}}{d t}=\frac{f}{\tau_{p}}\left(\vec{u}_{g}-\vec{u}_{p}\right)+\vec{g}
$$

\subsubsection{Conservation Equations for the Gas-Phase}

For an incompressible, constant viscosity flow in a Cartesian coordinate system the equations for conservation of mass and momentum are given, in tensor notation, by:

$$
\begin{aligned}
\frac{\partial u_{g i}}{\partial x_{i}} & =0 \\
\rho_{g} \frac{\partial u_{g i}}{\partial t}+\rho_{g} u_{g j} \frac{\partial u_{g i}}{\partial x_{j}} & =-\frac{\partial P}{\partial x_{i}}+\mu \frac{\partial^{2} u_{g i}}{\partial x_{j} \partial x_{j}}+F_{i}-F_{p i}
\end{aligned}
$$

In these equations $u_{g i}$ represents instantaneous gas-phase velocities in the $x_{i}$-direction. $P$ is the static pressure, and $F_{i}$ represents all external forces acting on the flow, including gravity. $F_{p i}$ is the particle source term per unit volume which accounts for the effects of the particulate-phase on the gas-phase.

For turbulent flows, the instantaneous value of any dependent variable. $O$. can he expressed as the sum of a mean and a fluctuating component, $\dot{\phi}=\bar{\phi}+\phi^{\prime}$, where $\bar{o}$ and $o^{\prime}$ represent the mean and fluctuating components respectively. In a process referred to as Reynolds averaging, the mean and fluctuating values are substituted into the mass and momentum equations and time-averaged. This yields the following equations:

$$
\begin{aligned}
\frac{\partial U_{g i}}{\partial x_{i}} & =0 \\
\rho_{g} \frac{\partial U_{g i}}{\partial t}+\rho_{g} U_{g j} \frac{\partial U_{g i}}{\partial x_{j}} & =-\frac{\partial \bar{P}}{\partial x_{i}}+\mu \frac{\partial^{2} U_{g i}}{\partial x_{j} \partial x_{j}}-\rho_{g} \frac{\partial \overline{u_{g i}^{\prime} u_{g j}^{\prime}}}{\partial x_{j}}+\overline{F_{i}}-\overline{F_{p i}}
\end{aligned}
$$

where $U_{g i}$ is the time-averaged fluid velocity (the overbar has been dropped for simplicity). The terms represented in tensor notation by the third term on the right-hand side of Eq. (2.11) are called the Reynolds stresses. The Reynolds stresses represent the mean rate of transport of momentum by turbulent fluctuations. 


\subsubsection{Turbulent Kinetic Energy}

An equation for the turbulent kinetic energy of the gas-phase, including the effect of the particulate-phase on the gas-phase is obtained by subtracting the expression for the mean kinetic energy from the expression for the turbulent kinetic energy (see Appendix A for a detailed derivation). Assuming incompressible, Stokian, constant viscosity flow: the equation for turbulent kinetic energy is given by:

$$
\begin{aligned}
& \underbrace{\rho_{g} \frac{\partial k}{\partial t}+\rho_{g} U_{g j} \frac{\partial k}{\partial x_{j}}}_{I}=-\underbrace{\rho_{g} \overline{u_{g i}^{\prime} u_{g j}^{\prime}} \frac{\partial U_{g j}}{\partial x_{i}}}_{I I}-\underbrace{\rho_{g} \frac{\partial}{\partial x_{i}} \overline{u_{g i}^{\prime}\left(\frac{P^{\prime}}{\rho_{g}}+k\right)}}_{I I I} \\
& +\underbrace{\mu \frac{\partial}{\partial x_{i}} \overline{u_{g j}^{\prime}\left(\frac{\partial u_{g i}^{\prime}}{\partial x_{j}}+\frac{\partial u_{g j}^{\prime}}{\partial x_{i}}\right)}}_{I V}-\underbrace{\frac{\mu}{2} \overline{\left(\frac{\partial u_{g i}^{\prime}}{\partial x_{j}}+\frac{\partial u_{g j}^{\prime}}{\partial x_{i}}\right)}}_{V} \\
& -\underbrace{\overline{u_{g i}^{\prime} \bar{F}_{p i}^{\prime}}}_{V I}
\end{aligned}
$$

Term (I) is the total change of the gas-phase kinetic energy. Term (II) represents the production of turbulent kinetic energy through the turbulent stresses. Term (III) is the diffusion of turbulent energy through pressure and velocity fluctuation interaction wirh the fluctuating velocity. Term (IV) represents the work done by turbulent viscous shear stresses. Term (V) is the viscous dissipation of turbulent kinetic energy, $\epsilon^{\prime}$. This term is always positive and thus acts as a sink in Eq. (2.12). Term (VI) represents the kinetic energy exchange between the gas and particle-phases. This term will be further discussed later in this section.

\subsubsection{Turbulent Dissipation Equation}

The turbulent dissipation rate, $\epsilon$, is derived in the same manner as the turbulent kinetic energy equation. However, since the algebra is quite involved, only the procedure will be outlined here [40]:

- Subtract the instantaneous momentum equation, Eq. (2.9), from the mean momentum equation, Eq.(2.11),

- differentiate the resulting equation with respect to $x_{k}$,

- multiply by $\nu \frac{\partial u_{g i}^{\prime}}{\partial x_{k}}$, 


\section{- time-average.}

An order of magnitude analysis is then performed and higher order terms neglected. The resulting equation is [40]:

$$
\begin{aligned}
& \frac{\partial \epsilon}{\partial t}+U_{g j} \frac{\partial \epsilon}{\partial x_{j}}=-2 \nu \frac{\partial U_{g i}}{\partial x_{j}}\left(\overline{\frac{\partial u_{g i}^{\prime}}{\partial x_{k}} \frac{\partial u_{g j}^{\prime}}{\partial x_{k}}}+\overline{\frac{\partial u_{g k}^{\prime}}{\partial x_{j}} \frac{\partial u_{g k}^{\prime}}{\partial x_{i}}}\right)-2 \nu \overline{\frac{\partial u_{g i}^{\prime}}{\partial x_{k}} \frac{\partial u_{g j}}{\partial x_{k}} \frac{\partial u_{g i}^{\prime}}{\partial x_{j}}} \\
& -\frac{\nu}{2} \frac{\partial}{\partial x_{j}} \overline{u_{g j}^{\prime}\left(\frac{\partial u_{g i}^{\prime}}{\partial x_{j}}+\frac{\partial u_{g j}^{\prime}}{\partial x_{i}}\right)^{2}}-2 \nu^{2} \overline{\frac{\partial^{2} u_{g i}^{\prime}}{\partial x_{j} \partial x_{k}} \frac{\partial^{2} u_{g i}^{\prime}}{\partial x_{j} \partial x_{k}}} \\
& +2 \nu \overline{\frac{\partial u_{g i}^{\prime}}{\partial x_{k}} \frac{\partial F_{p i}^{\prime}}{\partial x_{k}}}
\end{aligned}
$$

It is the last term of Eq. (2.13) that accounts for the two-way coupling betreen the phases.

\subsubsection{Coupling Between the Phases}

To account for the effect of the particulate-phase on the gas-phase an extra term. the particle source term, $F_{p}$, is included in the instantaneous momentum equation. Eq. (2.9). After Reynolds averaging, the mean momentum equation including the particle source has the form given in Eq. (2.11):

$$
\rho_{g} \frac{\partial U_{g i}}{\partial t}+\rho_{g} U_{g j} \frac{\partial U_{g i}}{\partial x_{j}}=-\frac{\partial \bar{P}}{\partial x_{i}}+\mu \frac{\partial^{2} U_{g i}}{\partial x_{j} \partial x_{j}}-\rho_{g} \frac{\partial \overline{u_{g i}^{\prime} u_{g j}^{\prime}}}{\partial x_{j}}+\overline{F_{i}}-\overline{F_{p i}}
$$

The average particle source term, $\overline{F_{p i}}$, is the force per unit volume exerted by the particles on the fluid. It is a function of the relative mean velocity between the particle and gasphases. The resulting slip leads to a hydrodynamic drag force, which, assuming Stokes flow $\left(R e_{p}<<1, f=1\right)$, is given by:

$$
\overline{F_{p i}}=\frac{\alpha}{\tau_{p}}\left(U_{g i}-U_{p i}\right)
$$

where $\alpha$ is the concentration of the particles defined as the mass of particles per unit volume.

In the turbulent kinetic energy equation, $\mathrm{Eq} .(2.12)$, it is the fluctuating component 
of the particle source term which governs the energy exchange between the phases.

$$
\begin{aligned}
F_{p i}^{\prime} & =\frac{\alpha}{\tau_{p}}\left(u_{g i}^{\prime}-u_{p i}^{\prime}\right) \\
\overline{u_{g i}^{\prime} F_{p i}^{\prime}} & =\frac{\alpha}{\tau_{p}}\left(\overline{u_{g i}^{\prime} \overline{u_{g i}^{\prime}}}-\overline{u_{g i}^{\prime} u_{p i}^{\prime}}\right)
\end{aligned}
$$

The term $\overline{u_{g i}^{\prime} F_{p i}^{\prime}}$ will act to decrease the gas-phase turbulent kinetic energy when the particles have smaller fluctuating velocities than the gas-phase. Conversely, if. as a result of initial conditions, the particulate-phase fluctuating velocities are larger than those of the gas-phase, $\overline{u_{g i}^{\prime} F_{p i}^{\prime}}$ will be negative and act as a source of turbulent kinetic energ::

The magnitude of $\overline{u_{g i}^{\prime} F_{p i}^{\prime}}$ is dependent on the concentration of the particles in the flow, $\alpha$, the relative fluctuating velocities between the phases and the particle response time. Recall from earlier in this section that the particle response time is a measure of the particles' ability to follow the turbulent fluctuations of the gas-phase. For small values of $\tau_{p}$, the particles will behave like fluid particles. In this limit, $\overline{u_{g i}^{\prime} u_{p i}^{\prime}}$ will equal $\overline{u_{g i}^{\prime} u_{g i}^{\prime}}$, causing $\overline{u_{g i}^{\prime} F_{p i}^{\prime}}$ to equal zero. For a large value of $\tau_{p}$; the particles will not respond to the turbulent fluctuations and $u_{p i}^{\prime}$ will equal zero. Thus, $\overline{u_{g i}^{\prime} F_{p i}^{\prime}}$ will approach zero. As discussed earlier, experimental trends suggest that large particles (i.e. large $\tau_{p}$ ) will cause an increase in the gas-phase turbulence through wake effects. This trend is not reflected in this source term; it is through other terms that an increase in turbulence intensity is achieved.

It is the last term of the turbulent dissipation equation, Eq. (2.13), which is responsible for communication with particle-phase:

$$
S_{\epsilon}=2 \nu \overline{\frac{\partial u_{g i}^{\prime}}{\partial x_{k}} \frac{\partial F_{p i}^{\prime}}{\partial x_{k}}}
$$

Substituting the expression for $F_{p i}^{\prime}$, the additional source term to account for coupling between the phases in the turbulent dissipation equation is given by,

$$
S_{\epsilon}=\frac{2}{\tau_{p}} \alpha\left[\nu\left[\frac{\partial u_{g i}^{\prime}}{\partial x_{k}}\left(\frac{\partial u_{g i}^{\prime}}{\partial x_{k}}-\frac{\partial u_{p i}^{\prime}}{\partial x_{k}}\right)\right]\right.
$$

Therefore, if the fluctuating gas velocity is greater than the fluctuating velocity of the particles then the rate of dissipation will increase. Likewise, if the fluctuating particle velocity is greater than the fluctuating gas velocity then the rate of dissipation will be decreased. 


\subsection{Review of Existing Models}

\subsubsection{Lagrangian versus Eulerian Formulation}

There are two approaches commonly used for modeling particle-laden flows: Lagrangian and Eulerian formulations. In an Eulerian formulation the gas and particle-phase are treated as two interactive fluids. The effect of turbulence modulation is included as extra terms in the continuum equations for both phases. The Lagrangian formulation tracks individual particles through the flow field. The particles are treated as sources of momentum and energy to the gas-phase. Both formulations are discussed below in more detail.

In the Eulerian formulation the continuity and momentum equations for the continuous phase are obtained through Reynolds averaging of the instantaneous equation (Section (2.2.2)). The governing equations for the particulate-phase are given by the 'dusty gas' equations of Marble (as given in [1]).

$$
\begin{aligned}
\frac{\partial \rho_{s}}{\partial t}+\frac{\partial \rho_{s} u_{p j}}{\partial x_{j}} & =0 \\
\frac{\partial\left(\rho_{s} u_{p i}\right)}{\partial t}+\frac{\partial\left(\rho_{s} u_{p j} u_{p i}\right)}{\partial x_{j}} & =-F_{p i}
\end{aligned}
$$

where $\rho_{s}$ is the particulate-phase loading. The particulate-phase loading is given by:

$$
\rho_{s}=\rho_{p} \theta
$$

where $\rho_{p}$ is the material density of the particle and $\theta$ is the particulate-phase rolume fraction. Applying Reynolds averaging and neglecting triple correlations yields the following equations:

$$
\begin{aligned}
\frac{\partial \overline{\rho_{s}}}{\partial t}+\frac{\partial \overline{\rho_{s} U_{p j}}}{\partial x_{j}}+\frac{\partial \overline{\rho_{s}^{\prime} u_{p j}^{\prime}}}{\partial x_{j}} & =0 \\
\frac{\partial\left(\overline{\rho_{s} U_{p i}}\right)}{\partial t}+\frac{\partial\left(\rho_{s}^{\prime} u_{p i}^{\prime}\right)}{\partial t}+\frac{\partial}{\partial x_{j}}\left(\overline{\rho_{s} U_{p i} U_{p j}}\right) & =-\frac{\partial}{\partial x_{j}}\left(\overline{\rho_{s}} \overline{u_{p i}^{\prime} u_{p j}^{\prime}}\right) \\
-\frac{\partial}{\partial x_{j}}\left(\overline{\rho_{s}^{\prime} u_{p i}^{\prime}} U_{p j}\right) & -\frac{\partial}{\partial x_{j}}\left(\overline{\rho_{s}^{\prime} u_{p j}^{\prime}} U_{p i}\right)-\overline{F_{p i}}
\end{aligned}
$$


The second-order correlations are modeled using a gradient diffusion type model $[-1]$.

$$
\begin{aligned}
& \overline{u_{p i}^{\prime} u_{p j}^{\prime}}=\nu_{p}\left(\frac{\partial \overline{u_{p i}}}{\partial x_{j}}+\frac{\partial \overline{u_{p j}}}{\partial x_{i}}\right)-\frac{2}{3} \delta_{i j}\left(\frac{1}{2} \overline{u_{p i}^{\prime} u_{p i}^{\prime}}+\nu_{p} \frac{\partial \overline{u_{p i}}}{\partial x_{i}}\right) \\
& \overline{\rho_{s}^{\prime} u_{p i}^{\prime}}=-D_{p} \frac{\partial \overline{\rho_{s}}}{\partial x_{i}}
\end{aligned}
$$

where $\nu_{p}$ is the effective turbulent kinematic viscosity for the particle-phase and $D_{p}$ is the turbulent diffusivity.

The turbulent diffusivity coefficient is one of the weaknesses in the Eulerian approach as there is debate in the literature concerning the selection of its value. Another inherent difficulty in this approach is the introduction of diffusion since this changes the equations from parabolic to elliptic. This causes the solution to the particle equations to become a boundary value problem which is contrary to the physics of particulate motion [6].

The Lagrangian formulation involves tracking individual particles through the flow field. The particles are tracked using the particle equation of motion, Eq. (2.3). In order to solve the particle equation of motion it is required that the fluid's velocity be knorn for every position of the particle. This requires a complete space-time representation of the gas-phase. The basic method by which this is achieved is as follows: The fluid fiow field is calculated assuming no particles are present (all values of $u_{g i}$ are obtained). The particle equation of motion is then solved for the particle's velocity and from this the trajectories calculated.

The underlying assumption of the Lagrangian formulation is that flows are sufficiently dilute so that there is no interaction between the two phases. In order to account for two-way coupling a method based on the particle-source-in-cell method of Crowe et al [9] is used. This is an iterative approach that involves treating the particles as sources of momentum and energy to the continuous phase. These source are calculated for each cell visited by the particle and added to the continuous phase equations in the next iteration.

The primary drawback to the Lagrangian approach is that many particles must be injected in order to determine statistically average behavior. This can involve large computational time. The Lagrangian approach does, however, preserve the underlying physics of two-phase flows. It also avoids the gradient-diffusion assumption. eliminating the need to select a turbulent diffusivity coefficient.

The present work will focus on Lagrangian models. A stochastic separated flow model will be used to solve for the particulate-phase, the two-equation $k$ - model will be used predict the gas-phase, and various models will be investigated to predict the turbulence 
modulation.

\subsubsection{Particulate-Phase Model}

The Stochastic Separated Flow (SSF) model used to predict the particle trajectories in the turbulent flow field is based on that of Shuen, Chen and Faeth [40]. This model assumes that the particles interact with a turbulent flow field that is made up of a series of characteristic eddies. Each eddy has associated with it a fluctuating velocity. $u_{g}^{\prime}$. a lifetime, $\tau_{e}$ and a characteristic length, $l_{e}$.

When a particle enters an eddy the fluctuating velocity for that eddy is added to the local mean fluid velocity to obtain the instantaneous fluid velocity used in the particle equation of motion. This velocity is held constant until one of two conditions is met: either the particle travels across the eddy or the eddy dissipates. When one of these conditions is met the particle is assumed to enter a new eddy which has new values of $u_{g}^{\prime}, \tau_{e}$ and $l_{e}$.

The turbulent field is assumed to be isotropic and possess a Gaussian probability distribution in the fluctuating velocity. The standard deviation, $\sigma_{i i}$, of the fluctuating velocity is given by,

$$
\sigma_{i i}^{2}=\left(\frac{2 k}{3}\right)=\overline{u_{g i}^{\prime 2}}
$$

To account for the randomness of the turbulence, $u_{g i}^{\prime}$ is determined through a random sampling of the Gaussian probability distribution such that,

$$
u_{g i}^{\prime}=\Gamma \sqrt{\frac{2}{3} k}
$$

where $\Gamma$ is a normally distributed random number. This allows $u_{g i}^{\prime}$ to take on a different value for each eddy that is encountered by the particle.

The particle is assumed to interact with the eddy for an interaction time. $\tau_{\text {int }}$. The interaction time is the minimum of the eddy lifetime, $\tau_{e}$, or the time required for tha particle to traverse the eddy, $\tau_{\ell r}$. It is further assumed that the characteristic size of the eddy is the dissipation length scale, $l_{e}$. Thus,

$$
\begin{aligned}
\tau_{i n t} & =\min \left(\tau_{e}, \tau_{t r}\right) \\
l_{e} & =\frac{C_{\mu}{ }^{3 / 4} k^{3 / 2}}{\epsilon}
\end{aligned}
$$




$$
\begin{aligned}
\tau_{e} & =\frac{l_{e}}{\sqrt{\frac{2}{3} k}} \\
\tau_{t r} & =-\tau_{p} \ln \left(1-\frac{l_{e}}{\left(\tau_{p}\left|\overrightarrow{u_{g}}-\overrightarrow{u_{p}}\right|\right)}\right)
\end{aligned}
$$

where $C_{\mu}=0.09$.

Variations on this SSF model have been proposed by researchers including Gosman and Ionnides [15], Zhou and Leschziner [48][49], and Chen and Crowe [5].

\subsubsection{Gas-Phase Model}

The majority of models that account for turbulence modulation in gas-solid flows are based on the $k-\epsilon$ model developed by Launder and Spalding (1974) [21].

The $k-\epsilon$ model is a widely used two-equation turbulence model that is used to approximate the Reynolds stress terms that appear in the time-averaged Navier-Stokes equations. The turbulent kinetic energy, $k$, and the dissipation rate, $\epsilon$, are defined as:

$$
\begin{aligned}
k & =\frac{1}{2} \overline{u_{g i}^{\prime} u_{g i}^{\prime}} \\
\epsilon & =\nu \frac{\partial u_{g i}^{\prime}}{\partial x_{k}} \frac{\partial u_{g i}^{\prime}}{\partial x_{k}}
\end{aligned}
$$

Using the gradient diffusion hypothesis the Reynolds stresses can be modeled for incompressible flows as:

$$
-\rho_{g} \overline{u_{g i}^{\prime} u_{g j}^{\prime}}=\mu_{t}\left(\frac{\partial U_{g i}}{\partial x_{j}}+\frac{\partial U_{g j}}{\partial x_{i}}\right)-\frac{2}{3} \rho_{g} \delta_{i j} k
$$

where $\mu_{t}$ is the turbulent viscosity;

$$
\mu_{t}=C_{\mu} \rho_{g} \frac{k^{2}}{\epsilon}
$$

and $C_{\mu}$ is a constant.

Applying this model to the mass and momentum equations gives the following result:

$$
\frac{\partial U_{g i}}{\partial x_{i}}=0
$$




$$
\rho_{g} \frac{\partial U_{g i}}{\partial t}+\rho_{g} U_{g j} \frac{\partial U_{p i}}{\partial x_{j}}=-\frac{\partial \bar{P}}{\partial x_{i}}+\frac{\partial}{\partial x_{j}}\left(\mu_{\mathrm{eff}}\left(\frac{\partial U_{g i}}{\partial x_{j}}+\frac{\partial U_{g j}}{\partial x_{i}}\right)\right)+\overline{F_{i}}-\overline{F_{p i}}
$$

where $\mu_{\mathrm{eff}}=\mu+\mu_{t}$.

Thus, if $k$ and $\epsilon$ can be determined, the Reynolds stresses can be computed and the time-averaged Navier-Stokes equations can be solved.

The turbulent kinetic energy equation, Eq. (2.12), and the dissipation equation. Eq. (2.13), derived in Section 2.2, form the basis for the $k-\epsilon$ model. The modeled forms of these equations, as presented by Launder and Spalding (1974) [21], are:

$$
\begin{aligned}
& \frac{D k}{D t}=\frac{1}{\rho_{g}} \frac{\partial}{\partial x_{k}}\left[\frac{\mu_{t}}{\sigma_{k}} \frac{\partial k}{\partial x_{k}}\right]+\frac{\mu_{t}}{\rho_{g}}\left(\frac{\partial U_{g i}}{\partial x_{k}}+\frac{\partial U_{g k}}{\partial x_{i}}\right) \frac{\partial U_{g i}}{\partial x_{k}}-\epsilon \\
& \frac{D \epsilon}{D t}=\frac{1}{\rho_{g}} \frac{\partial}{\partial x_{k}}\left[\frac{\mu_{t}}{\sigma_{\epsilon}} \frac{\partial \epsilon}{\partial x_{k}}\right]+\frac{C_{1} \mu_{t}}{\rho} \frac{\epsilon}{k}\left(\frac{\partial U_{g i}}{\partial x_{k}}+\frac{\partial U_{g k}}{\partial x_{i}}\right) \frac{\partial U_{g i}}{\partial x_{k}}-C_{2} \frac{\epsilon^{2}}{k}
\end{aligned}
$$

where $\sigma_{k}, \sigma_{\epsilon}, C_{1}$ and $C_{2}$ are constants. Suggested values for the constants used in the $k-\epsilon$ model are presented below [21]:

\begin{tabular}{lllll}
\hline$C_{\mu}$ & $C_{1}$ & $C_{2}$ & $\sigma_{k}$ & $\sigma_{\epsilon}$ \\
\hline 0.09 & 1.44 & 1.92 & 1.0 & 1.3 \\
\hline
\end{tabular}

To account for turbulence modulation by the particles, the additional source terms clerived in Section 2.2 must be added to the model. Thus, the $k-\epsilon$ model with additional source terms is as follows:

$$
\begin{aligned}
& \frac{D k}{D t}=\frac{1}{\rho_{g}} \frac{\partial}{\partial x_{k}}\left[\frac{\mu_{t}}{\sigma_{k}} \frac{\partial k}{\partial x_{k}}\right]+\frac{\mu_{t}}{\rho_{g}}\left(\frac{\partial U_{g i}}{\partial x_{k}}+\frac{\partial U_{g k}}{\partial x_{i}}\right) \frac{\partial U_{g i}}{\partial x_{k}}-\epsilon-S_{k} \\
& \frac{D \epsilon}{D t}=\frac{1}{\rho_{g}} \frac{\partial}{\partial x_{k}}\left[\frac{\mu_{t}}{\sigma_{\epsilon}} \frac{\partial \epsilon}{\partial x_{k}}\right]+\frac{C_{1} \mu_{t} \epsilon}{\rho_{g}} \frac{\epsilon}{k}\left(\frac{\partial U_{g i}}{\partial x_{k}}+\frac{\partial U_{g k}}{\partial x_{i}}\right) \frac{\partial U_{g i}}{\partial x_{k}}-C_{2} \frac{\epsilon^{2}}{k}+S_{\epsilon}
\end{aligned}
$$

where,

$$
\begin{aligned}
S_{k} & =\frac{1}{\rho_{g}} \frac{\alpha}{\tau_{p}}\left(\overline{u_{g i}^{\prime} u_{g i}^{\prime}}-\overline{u_{g i}^{\prime} u_{p i}^{\prime}}\right) \\
S_{\epsilon} & =\frac{1}{\rho_{g}} \frac{2}{\tau_{p}} \alpha\left[\nu \overline{\left.\frac{\partial u_{g i}^{\prime}}{\partial x_{j}}\left(\frac{\partial u_{g i}^{\prime}}{\partial x_{j}}-\frac{\partial u_{p i}^{\prime}}{\partial x_{j}}\right)\right]}\right.
\end{aligned}
$$


It is generally the correlations $\overline{u_{g i}^{\prime} u_{p i}^{\prime}}$ and $\overline{\frac{\partial u_{g i}^{\prime}}{\partial x_{j}} \frac{\partial u_{p i}^{\prime}}{\partial x_{j}}}$ that require additional modeling.

\subsubsection{Turbulence Modulation Models}

What follows is a discussion of the various types of turbulence modulation models found in the literature. Not all of these models are of a type that is easily implemented into a $k-\epsilon$ type model (Shuen et al $[40,41]$, Yuan and Michaelides [47], Kenning and Crowe [20]) and will therefore not be implemented in this investigation. These models do, however, provide insight into the problem of modelling turbulence modulation and warrant consideration here.

The main weakness in those models which can be implemented into a $k-\epsilon$ type model (Chen and Wood [4], Adeniji-Fashola and Chen [1], Tu and Fletcher [45], Mostafa and Mongia [30]) is their inability to predict and increase in the gas-phase turbulence intensity. They are discussed in further detail below.

A summary of the of the models reviewed including the experiments used to ralidate them is presented in Table 2.2 at the end of this section.

\section{Chen and Wood, 1985}

Chen and Wood [4] developed a simple model to predict the effects of turbulence modulation. In this model the correlation $\overline{u_{g i}^{\prime} u_{p i}^{\prime}}$ is modeled as,

$$
\overline{u_{g i}^{\prime} u_{p i}^{\prime}}=2 k \exp \left(-B_{k} \frac{\tau_{p}}{\tau_{e}}\right)
$$

where $\tau_{e}$ is the time scale of the most energetic eddy, $\tau_{e}=0.165 \frac{k}{\epsilon}$. The constant $B_{k}$ was assigned a value of $B_{k}=0.0825$ based on a previous model by Chen and Wood.

Thus the additional source term in the k-equation is given by:

$$
S_{k}=\frac{1}{\rho_{g}} \frac{2 k}{\tau_{p}} \alpha\left[1-\exp \left(-\frac{0 . \overline{5} \tau_{p} \epsilon}{k}\right)\right]
$$

The additional term in the dissipation equation was modeled by looking at the dissipative eddies. These are the eddies that contribute most to the correlation $\frac{\overline{\partial u_{q z}^{\prime}}}{\partial x_{j}} \frac{\partial u_{p z}^{\prime}}{\partial x_{j}}$. It was assumed that $u_{g i}^{\prime}$ and $u_{p i}^{\prime}$ are completely uncoupled on the time scale of the dissipative eddies, $\tau=\left(\frac{\nu}{\epsilon}\right)^{\frac{3}{2}}$, since $\tau_{p} \gg \tau$ in most turbulent flows. Thus the additional term 
in the dissipation equation reduces to

$$
S_{\epsilon}=-\frac{1}{\rho_{g}} \frac{2}{\tau_{p}} \alpha \epsilon
$$

This will not be correct for small particles where the values of $\tau_{p}$ approaches $\tau$.

Results calculated from the model, including turbulent kinetic energy and mean Reynolds Shear Stress, are in good agreement with the experimental data for the cases considered, however this model will always predict a decrease in the turbulence of the gas-phase and is therefore not applicable to flows involving large particles.

\section{Adeniji-Fashola and Chen, 1990}

Adeniji-Fashola and Chen [1] looked at turbulent gas-solid flows using both Eulerian and Lagrangian modeling approaches.

The Eulerian approach used by the authors is the same as that used by Chen and Wood (1985) [4]:

$$
\begin{aligned}
S_{k} & =\frac{1}{\rho_{g}} \frac{2 k}{\tau_{p}} \alpha\left[1-\exp \left(-\frac{0 . \bar{\delta} \tau_{p} \epsilon}{k}\right)\right] \\
S_{\epsilon} & =-\frac{1}{\rho_{g}} \frac{2}{\tau_{p}} \alpha \epsilon
\end{aligned}
$$

In the Lagrangian formulation an SSF model similar to that of Gosman and Ioannides (1983) [15] is used to solve for the particle motion. At this point the instantaneous particle: source properties are known from the stochastic formulation and the additional source terms appearing in the $k$ equation are exact and require no modeling. However. since the numerical procedure for these terms was not well established, Adeniji-Fashola and Chen chose to model the additional terms in the $k$ and $\epsilon$ equations in the same manner as used in the Eulerian approach.

Both formulations of the model were compared with experimental data from Tsuji et al [44]. The Lagrangian formulation was not very accurate at predicting the general trend of the turbulence modulation across the pipe. The Eulerian prediction was slightly more accurate, however the predicted modulation was still not in good agreement with the experimental data. Both formulations of this model will predict only an attenuation of gas-phase turbulence. 


\section{Tu and Fletcher, 1994}

Tu and Fletcher [45] proposed a model similar to that of Chen and Wood [4] but which also accounts for the effects of the loading ratio on turbulence modulation. As discussed in Section 2.1 it has been shown experimentally that for large particles as the loading ratio, $w$, is increased the magnitude of the augmentation of the turbulence is increased. For small particles the magnitude of the attenuation increases to a certain level and then decreases after a certain loading ratio has been reached.

To account for the effect of the loading ratio on the turbulence modulation $\mathrm{Tu}$ and Fletcher introduced the parameters $w$ and $n$ into a model of the same form as Chen and Wood's. The additional source terms are:

$$
\begin{gathered}
S_{k}=\frac{1}{\rho_{g}} \frac{2 k}{\tau_{p}} \alpha\left[1-\exp \left(-\frac{B_{k} \tau_{p}}{w^{n} \tau_{e}}\right)\right] \\
S_{\epsilon}=-\frac{1}{\rho_{g}} \frac{2 \epsilon}{\tau_{p}} \alpha\left[1-\exp \left(-\frac{B_{\epsilon} \tau_{p}}{w^{n} \tau_{e}}\right)\right] \\
n=0 \text { for } w \leq 1 \\
n=1 \text { for } w>1
\end{gathered}
$$

where $B_{k}=0.09, B_{\epsilon}=0.4$ (determined from the experimental data) and $\tau_{e}=\frac{k}{\epsilon}$.

Turbulence intensity results obtained from the model were compared to experimental data based on pipe flow (the same data used to obtain the constants $B_{k}$ and $B_{\epsilon}$ ). Good agreement was obtained in the outer region of the pipe, however the turbulence intensity was significantly under predicted near the pipe center. Again, this model will predict only a decrease in turbulence intensity in gas-solid flows.

\section{Mostafa and Mongia, 1988}

Mostafa and Mongia [30] formulated a model in which the turbulent correlation. $\overline{u_{g i}^{\prime} u_{p i}^{\prime}}$. is modeled based on the gas-phase turbulent kinetic energy, the Lagrangian time scale. $\tau_{L I}$, and the particle response time, $\tau_{p}$,

$$
\overline{u_{g i}^{\prime} u_{p i}^{\prime}}=2 k \frac{\tau_{L I}}{\tau_{L I}+\tau_{p}}
$$

where $\tau_{L I}$ is given by $\tau_{L I}=0.35 \frac{k}{\epsilon}$. The correlation $\frac{\partial u_{g i}^{\prime}}{\partial x_{j}} \frac{\partial u_{p i}^{\prime}}{\partial x_{j}}$ is modeled in a similar fashion. 
Therefore, assuming Stokes flow, the extra-terms in the turbulent energy and dissipation equations are:

$$
\begin{aligned}
& S_{k}=\frac{1}{\rho_{g}} \frac{2 k}{\tau_{p}} \alpha\left(1-\frac{\tau_{L I}}{\tau_{L I}+\tau_{p}}\right) \\
& S_{\epsilon}=-\frac{1}{\rho_{g}} C_{\epsilon 3} \frac{2 \epsilon}{\tau_{p}} \alpha\left(1-\frac{\tau_{L I}}{\tau_{L I}+\tau_{p}}\right)
\end{aligned}
$$

where the model constant $C_{\epsilon 3}=1.0$.

Results from the model were compared to experimental results for a two-phase turbulent round jet. The predictions were in good agreement with the experimental dara. When the present model was compared with the standard $k-\epsilon$ model it was shown that results obtained with this model were significantly better than those obtained with the $k-\epsilon$ model. This model was examined again by Mostafa et al (1989) [3I] using different values for the constants appearing in the $k-\epsilon$ model. Good agreement was shown with experimental data. This model will not predict an increase in turbulence.

\section{Shuen et al, 1983, 1985}

Shuen et al $[40,41]$ investigated the use of the instantaneous flow properties known from the SSF formulation to predict the turbulence modulation in two-phase flows. Lsing the instantaneous flow properties, all correlations are known for $S_{k}$, the turbulence energysource term. Therefore only the dissipation source term, $S_{\epsilon}$, requires modeling. This term is modeled as,

$$
S_{\epsilon}=2 C_{\epsilon 3} \nu_{t} \frac{\epsilon}{k}\left(\overline{\frac{\partial u_{g i}^{\prime}}{\partial x_{j}} \frac{\partial u_{g i}^{\prime}}{\partial x_{j}}}-\overline{\frac{\partial u_{g i}^{\prime}}{\partial x_{j}} \frac{\partial u_{p i}^{\prime}}{\partial x_{j}}}\right)
$$

where $\nu_{\iota}=C_{\mu} \frac{k^{2}}{\epsilon}, C_{\epsilon 3}$ is between 0.1 and 0.5 , and $C_{\mu}=0.09$. It was determined that predictions from the model are relatively insensitive to changes in $c_{\epsilon 3}$, however in both studies flows were too dilute to determine a value for $c_{\varepsilon 3}$. Furthermore, flows were too dilute to gain any significant insight into the ability of the SSF model formulation to predict turbulence modulation.

\section{Yuan and Michaelides, 1992}

Yuan and Michaelides [47] developed a simple model which attempts to account for both the augmentation and reduction of turbulence in two-phase flows. They identify two mechanisms for turbulence modulation: 
1. The transfer of energy from an eddy in order to accelerate a particle (responsible for turbulence reduction).

2. The flow velocity disturbance by a particle due to its wake or vortex shedding (responsible for turbulence production).

The total work done by the eddy on a particle, $W_{e}$, over the interval $\tau_{\text {int }}$ (the minimum of the eddy lifetime and the time it takes for the particle to cross the eddy) is defined as:

$$
W_{e}=\int_{0}^{\tau_{\text {int }}} P_{\epsilon} d t
$$

where $P_{\epsilon}$, the rate of energy dissipation, is the product of the drag force and the relative velocity given by,

$$
P_{\epsilon}=\frac{\pi C_{D} d^{2} \rho_{g}\left(u_{g}-u_{p}\right)^{2}\left|u_{g}-u_{p}\right|}{8}
$$

Yuan and Michaelides then derived from the particle equation of motion. Eq. (2.3). an approximate expression for the particle velocity during the time interval $\tau_{\text {int }}$.

$$
u_{p}=u_{p o}+\left(u_{g}-u_{p o}\right)\left[1-\exp \left(\frac{-f t}{\tau_{p}}\right)\right]
$$

where $f=\left(1+0.15 R e_{p}{ }^{0.687}\right)$. Integrating $P_{\epsilon}$ with respect to time gives,

$$
W_{e}=\frac{\pi}{12} d^{3} \rho_{p}\left(u_{g}-u_{p o}\right)^{2}\left[1-\exp \left(-\frac{2 f \tau_{\text {int }}}{\tau_{p}}\right)\right]
$$

The energy transfered from the particulate-phase to the gas-phase by the presence of particles is given by:

$$
\triangle E_{p}=\frac{\pi}{12} d^{2} \rho_{g}\left(u_{g}^{2}-u_{p}^{2}\right) f\left(l_{w}\right)
$$

where $l_{w}$ is a length scale representing the region behind the particle where the Huid velocity is close to that of the particle, such as the effective length of the wake or the length behind the particle where vortices are shed. Crowe [8] suggests that the term $\left(u_{g}{ }^{2}-u_{p}{ }^{2}\right)$ should have an absolute value sign, since a wake should always augment turbulence. 
A combination of the reduction and production terms gives an expression for the total turbulence modulation,

$$
\triangle E_{k}=-\frac{\pi}{12} d^{3} \rho_{p}\left(u_{g}-u_{p o}\right)^{2}\left[1-\exp \left(-\frac{2 f \tau_{i n t}}{\tau_{p}}\right)\right]+\frac{\pi}{12} d^{2} \rho_{g}\left(u_{g}{ }^{2}-u_{p}{ }^{2}\right) f\left(l_{w}\right)
$$

This model was not incorporated into the $k-\epsilon$ model, however good agreement was shown when the total change in turbulent kinetic energy was compared to experimental results obtained in pipe and jet flow.

\section{Kenning and Crowe, 1997}

Kenning and Crowe [20] proposed a physical model based on interparticle spacing to account for turbulence modulation in gas-solid flows. Two 'sources' of turbulence energy: are identified: $P_{i}$, the turbulence of the fluid in the absence of particles, or inherent turbulence, and $\boldsymbol{P}_{\boldsymbol{d}}$, the production of turbulence due to the presence of the dispersed phase. Similarly, two mechanisms for the dissipation of turbulence energy are defined: $\epsilon$, the viscous dissipation, and $\epsilon_{d}$, the retransmission of energy to the motion of solid particles.

In the absence of particles, the inherent turbulence production, $P_{i}$, is equal to the inherent dissipation (for equilibrium flows). Thus,

$$
P_{i}=\frac{k_{i}^{3 / 2}}{L_{\epsilon i}}
$$

where $k_{i}$ is the inherent turbulent kinetic energy of the flow and $L_{i}$ is the dissipation length scale of the flow without particles.

It is proposed that the average energy produced by the fluid in the presence of particles is based on the velocity difference between the particles and the fluid. Therefore.

$$
\overline{P_{d}}=\frac{f}{\tau_{p}}\left(U_{g}-U_{p}\right)^{2}
$$

where $f$ is the ratio of particle drag to Stokes' drag.

For the gas-particle flow considered, the dissipation due to the particles is small with respect to the production term, $\overline{\boldsymbol{P}}_{d}$. Therefore, $\epsilon_{d}$ is considered to be negligible.

The viscous dissipation is given by,

$$
\epsilon=\frac{k^{3 / 2}}{L_{h}}
$$


where $L_{h}$ represents the hybrid length scale of the flow.

There are various length scales that can be considered. For example. the integral length scale, $L$ is generally on the order of the pipe or jet diameter. For two-phase flows. significant length scales include particle diameter and average interparticle spacing. Consider a concentration of particles introduced into the flow with an average interparticle spacing less than the inherent dissipation length scale. These particles may break up the existing eddies so that the new dissipation length scale is proportional to the arerage interparticle spacing. Therefore, Kenning and Crowe chose to base the hybrid length scale on the inherent dissipation length scale, $L_{\epsilon i}$, and the average interparticle spacing, $\lambda$, where $\lambda / d_{p} \approx(\pi / 6 \phi)^{1 / 3}-1$, and $\phi$ represents the volume fraction of the particulate-phase. Thus,

$$
L_{h}=\frac{2 L_{\epsilon i} \lambda}{L_{\epsilon i}+\lambda}
$$

Performing an energy balance on the production and dissipation terms yields.

$$
\left(\overline{u_{g}}-\overline{u_{p}}\right)^{2} \frac{\rho_{p}}{\rho_{g}} \frac{f}{\tau_{p}}-\frac{k^{3 / 2}}{L_{h}}+\frac{k_{i}^{3 / 2}}{L_{\epsilon i}}=0
$$

Solving for $k / k_{i}$ and noting that $k / k_{i}$ equals $T_{i} / T_{i i}$, the ratio of the two-phase turbulence intensity to the inherent turbulence intensity gives the following equation for the fractional change in turbulence intensity:

$$
\frac{T_{i}-T_{i i}}{T_{i i}}=\left[\frac{L_{h}}{L_{i}}+\frac{L_{h}}{k_{i}^{3 / 2}} \frac{f\left(\overline{u_{g}}-\overline{u_{p}}\right)^{2}}{\tau_{p}} \frac{\rho_{p}}{\rho_{f}}\right]^{1 / 3}-1
$$

Again, this model was not incorporated into a $k-\epsilon$ model. Instead, the fractional change in turbulence intensity was compared with experimental data. Reasonable agreement was obtained, which suggests that interparticle spacing is an important length scale in twophase turbulent flows.

\subsection{Summary}

This chapter began with an overview of experimental work relating to turbulence modulation. Two parameters were identified as playing a role in turbulence modulation: The particle diameter and the loading ratio of the particles. Three particle diameter regimes were identified. Small particles $\left(d_{p}<250 \mu m\right)$ will cause a decrease in the turbulence intensity, intermediate particles $\left(250 \mu m<d_{p}<800 \mu m\right)$ can cause either an increase or a decrease and large particles $\left(d_{p}>800 \mu m\right)$ will cause an increase in turbulence intensity: 
Table 2.2: Summary of Models Reviewed

\begin{tabular}{|c|c|c|c|c|}
\hline Model & $\begin{array}{l}\text { Model } \\
\text { Formulation }\end{array}$ & $\begin{array}{l}\text { Validating } \\
\text { Experiment(s) }\end{array}$ & $\begin{array}{c}\overline{d_{p}} \\
(\mu \mathbf{m})\end{array}$ & $\begin{array}{c}\text { Loading } \\
\text { Ratio }\end{array}$ \\
\hline \multirow[t]{3}{*}{ Chen and Wood, 1985} & \multirow[t]{3}{*}{ Eulerian } & \multirow{2}{*}{$\begin{array}{l}\text { Wall et al, } 1982 \\
\text {-no turbulence } \\
\text { data available }\end{array}$} & 98 & $\begin{array}{l}0.50 \\
0.22\end{array}$ \\
\hline & & & $\overline{33}$ & 0.22 \\
\hline & & Modarress et al, 1984 & 50 & 0.32 \\
\hline $\begin{array}{l}\text { Adeniji-Fashola } \\
\text { and Chen, } 1990\end{array}$ & $\begin{array}{l}\text { Lagrangian } \\
\text { \& Eulerian }\end{array}$ & Tsuji et al, 1984 & 200 & $\begin{array}{l}3.2 \\
2.1 \\
1.0\end{array}$ \\
\hline Tu and Fletcher, 1994 & Lagrangian & Tsuji et al, 1984 & 200 & 3.2 \\
\hline $\begin{array}{l}\text { Mostafa and Mongia, } \\
1988\end{array}$ & Lagrangian & Shuen et al, 1983 & 119 & $\begin{array}{l}0.66 \\
0.20 \\
\end{array}$ \\
\hline $\begin{array}{l}\text { Mostafa et al, } \\
1989\end{array}$ & Lagrangian & Mostafa et al, 1989 & 105 & $\begin{array}{l}1.0 \\
0.2\end{array}$ \\
\hline \multirow[t]{3}{*}{ Shuen et al, 1983} & \multirow[t]{3}{*}{ Lagrangian } & \multirow[t]{3}{*}{ Shuen et al, 1983} & 207 & 0.66 \\
\hline & & & 119 & $\begin{array}{l}0.66 \\
0.20 \\
\end{array}$ \\
\hline & & & 79 & 0.20 \\
\hline \multirow[t]{3}{*}{ Shuen et al, 1985} & \multirow[t]{3}{*}{ Lagrangian } & \multirow[t]{3}{*}{ Shuen et al, 1985} & 207 & 0.66 \\
\hline & & & 119 & $\begin{array}{l}0.66 \\
0.20\end{array}$ \\
\hline & & & 79 & 0.20 \\
\hline \multirow{7}{*}{$\begin{array}{l}\text { Yuan and Michaelides, } \\
1992\end{array}$} & \multirow[t]{7}{*}{$\mathrm{n} / \mathrm{a}$} & \multirow[t]{4}{*}{ Tsuji et al, $1 \overline{984}$} & 3000 & $\mathrm{n} / \mathrm{a}$ \\
\hline & & & 1000 & $\mathrm{n} / \mathrm{a}$ \\
\hline & & & 500 & $n / a$ \\
\hline & & & 200 & $\mathrm{n} / \mathrm{a}$ \\
\hline & & Modarress et al, 1984 & 50 & $\begin{array}{l}0.85 \\
0.32\end{array}$ \\
\hline & & \multirow[t]{2}{*}{$\begin{array}{l}\text { Levy and Lockwood, } \\
1981\end{array}$} & 215 & $\begin{array}{l}3 . \overline{30} \\
1.1 \pm \\
\end{array}$ \\
\hline & & & 1050 & 3.5 \\
\hline \multirow[t]{6}{*}{ Kenning and Crowe, } & \multirow[t]{6}{*}{$\mathrm{n} / \mathrm{a}$} & $\begin{array}{l}\text { Levy and Lockwood, } \\
1981\end{array}$ & 800 & $\mathrm{n} / \mathrm{a}$ \\
\hline & & \multirow[t]{4}{*}{ Tsuji et al, $1 \overline{984}$} & 3000 & $\mathrm{n} / \mathrm{a}$ \\
\hline & & & 1000 & $\mathrm{n} / \mathrm{a}$ \\
\hline & & & 500 & $\mathrm{n} / \mathrm{a}$ \\
\hline & & & 200 & $\mathrm{n} / \mathrm{a}$ \\
\hline & & Lee and Durst, 1982 & 800 & $\mathrm{n} / \mathrm{a}$ \\
\hline
\end{tabular}


It was found that for large particles as the loading ratio of the particles increases there is an increase in the turbulence intensity of the flow. For small particles, an increase in loading ratio will initially reduce the turbulence intensity but a further increase in loading ratio will bring the turbulence intensity back up.

Four mechanisms by which turbulence modulation occurs were proposed. The first way in which the turbulent flow field can be modified is through the drag force between the gas and particle-phase. This leads to a reduction of turbulence intensity: Increased dissipation can also occur when a particle collides with a similar-sized eddy. This can cause the eddy to break up into smaller eddies, increasing the energy dissipation in the flow. Turbulence intensity can be increased through wake formation and vortex shedding caused by the particles. This typically occurs for large particles. Finally, the crossing trajectory effect, which occurs as a result of the particles falling through the eddies and dragging the mean gas flow along, can also cause an increase in turbulence intensity:

The mean velocity of the gas-phase is also affected by particle size and loading ratio. It is not clear how particle size affects the mean velocity, an increase or decrease can occur within a given size regime. Varying the loading ratio has the same effect on the mean momentum as it did on the turbulent kinetic energy.

The fundamental equations pertaining to turbulence modulation were presented. These include the particle equation motion and the conservation equations for the gasphase including a detailed derivation of the turbulent kinetic energy equation. The source term that accounts for coupling between the phases was derived for the mean momentum equation, and the turbulent kinetic energy and dissipation equations.

Finally, a review of the models used in this work were presented. The stochastic separated flow model of Shuen, Chen and Faeth [40] is used to predict the particle dispersion in the turbulent flow. The $k-\epsilon$ model of Launder and Spalding [21] was used to predict the turbulent flow field. This model forms the basis of this investigation: it is through the $k-\epsilon$ model that turbulence modulation is modeled. Existing turbulence modulation models were presented. The models of Chen and Wood [4], Tu and Fletcher [45] and Mostafa and Mongia [30] are the focus of this research. 


\section{Chapter 3}

\section{The Model of Lightstone and Hodgson}

This chapter introduces the model of Lightstone and Hodgson. The exact expression for the gas-particle fluctuating velocity correlation is derived in full and motivation for the development of the current model discussed. The model is then presented and examinerl for limiting cases.

\subsection{Motivation}

The $k-\epsilon$ model is used for modeling turbulent flows in most commercially arailable computational fluid dynamics codes. As such, the focus of this investigation is on those turbulence modulation models that can be implemented as source terms to the $k-\epsilon$ model, several of which are presented in Section 2.3.

As presented in Section 2.2.5, the exact expression for the source term to the $k$. equation is:

$$
\overline{u_{g i}^{\prime} F_{p i}^{\prime}}=\frac{\alpha}{\tau_{p}}\left(\overline{u_{g i}^{\prime} u_{g i}^{\prime}}-\overline{u_{g i}^{\prime} u_{p i}^{\prime}}\right)
$$

In keeping with the $k-\epsilon$ model, $\overline{u_{g i}^{\prime} u_{g i}^{\prime}}=2 k$. It is the correlation between the fluctuating velocity of the gas-phase and the fluctuating velocity of the particle-phase. $\overline{u_{g}^{\prime} u_{p}^{\prime}}$, that must be modeled. As a first step towards developing a model, the exact expression for this correlation is derived.

The particle equation of motion for Stokes' flow was presented in Section 2.2.1 and is repeated here:

$$
\frac{d \vec{u}_{p}}{d t}=\frac{1}{\tau_{p}}\left(\vec{u}_{g}-\vec{u}_{p}\right)+\vec{g}
$$

Expressing the gas-phase velocity in terms of its mean and fluctuating components 
$\left(u_{g}=U_{g}+u_{g}^{\prime}\right)$, substituting into Eq.(3.2) and rearranging gives:

$$
\frac{d u_{p}}{d t}+\frac{u_{p}}{\tau_{p}}=\frac{u_{g}^{\prime}}{\tau_{p}}+\left(g+\frac{U_{g}}{\tau_{p}}\right)
$$

Multiply Eq.(3.3) by an integrating factor in the form $e^{\frac{\eta}{\tau_{p}}}$, where $\eta$ is a dummy. variable for $t$, and integrate from 0 to $t$ :

$$
\begin{aligned}
\int_{0}^{t} \frac{d}{d \eta}\left(u_{p} e^{\frac{\eta}{\tau_{p}}}\right) d \eta & =\int_{0}^{t} e^{\frac{\eta}{\tau_{p}}} \frac{u_{g}^{\prime}}{\tau_{p}} d \eta+\int_{0}^{t} e^{\frac{\eta}{\tau_{p}}}\left(g+\frac{U_{g}}{\tau_{p}}\right) d \eta \\
u_{p}(t) e^{\frac{t}{\tau_{p}}}-u_{p}(0) & =\int_{0}^{t} e^{\frac{\eta}{\tau_{p}}} \frac{u_{g}^{\prime}}{\tau_{p}} d \eta+\int_{0}^{t} e^{\frac{\eta}{\tau_{p}}}\left(g+\frac{U_{g}}{\tau_{p}}\right) d \eta \\
u_{p}(t) & =\frac{e^{\frac{-t}{\tau_{p}}}}{\tau_{p}} \int_{0}^{t} e^{\frac{\eta}{\tau_{p}}} u_{g}^{\prime}(\eta) d \eta+e^{\frac{-t}{\tau_{p}}} \int_{0}^{t} e^{\frac{\eta}{\tau_{p}}}\left(g+\frac{U_{g}}{\tau_{p}}\right) d \eta+e^{\frac{-t}{\tau_{p}}} u_{p}(0)
\end{aligned}
$$

Multiplying Eq.(3.4) by $u_{g}^{\prime}(t)$ and time-averaging yields:

$$
\overline{u_{g}^{\prime}(t) u_{p}(t)}=\frac{e^{\frac{-t}{\tau_{p}}}}{\tau_{p}} \int_{0}^{t} e^{\frac{\eta}{\tau_{p}}} \overline{u_{g}^{\prime}(t) u_{g}^{\prime}(\eta)} d \eta+e^{\frac{-t}{\tau_{p}}} \overline{u_{g}^{\prime}(t) u_{p}(0)}
$$

Note that $\overline{u_{g}^{\prime}(t) u_{p}(t)}$ is equal to $\overline{u_{g}^{\prime}(t) u_{p}^{\prime}(t)}$ since $\overline{u_{g}^{\prime}(t) U_{p}}=0$.

An expression for $\overline{u_{g}^{\prime}(t) u_{p}^{\prime}(\eta)}$ is required to complete the new model formulation. This quantity can be written as a correlation function, $R_{g p}(t-\eta)$, where $R_{g p}=\overline{u_{g}^{\prime}(t) u_{g}^{\prime}(\eta)} / \overline{u_{g}^{\prime 2}}$. It is important to note that the correlation $R_{g p}$ is the correlation of the change in time and position experienced by a fluid particle over a timestep $(t-\eta)$. Consider the situation shown in Figure 3.1. At time $t=0$ a particle is released from point $x_{1}$. Because of inertia and crossing trajectory effects the particle is unable to exactly follow the fluid. so at a time $t=\tau$ it will have traveled along a path to a position $x_{2}$ while the fluid particle will have traveled along a different path to a position $x_{3}$. The autocorrelation function. $R_{g p}$. is the correlation of the fluid velocity along the particle path.

This correlation is approximated as:

$$
R_{g p}=\frac{\overline{u_{g}^{\prime}(t) u_{g}^{\prime}(\eta)}}{\overline{u_{g}^{\prime 2}}}=e^{\frac{-(t-n)}{\tau^{\circ}}}
$$

where $\tau^{*}$ is the integral time scale of the fluid velocity along the particle path. Substituting 


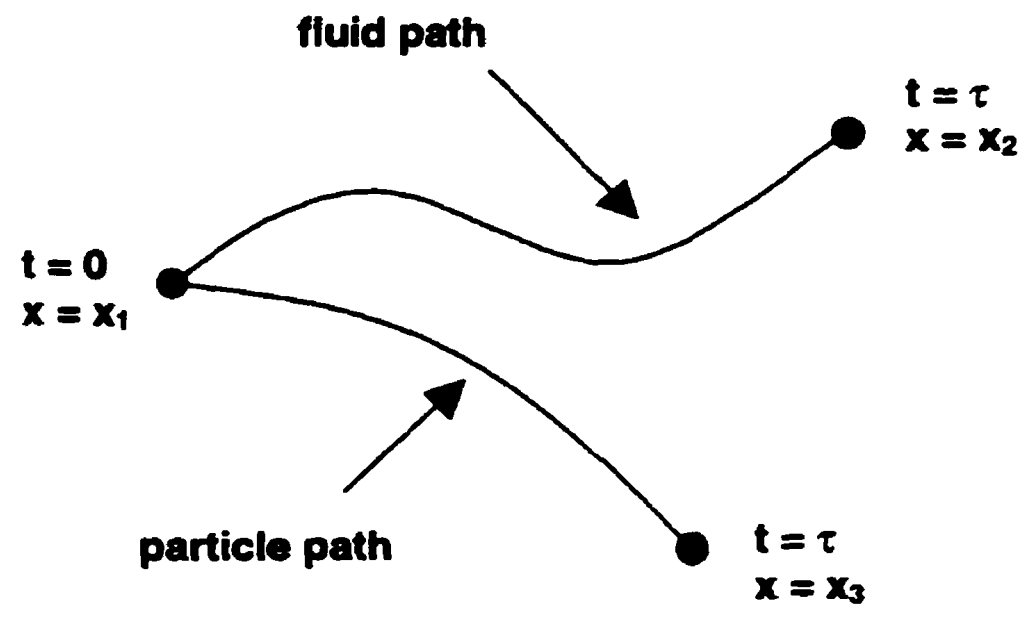

Figure 3.1: Particle and Fluid Trajectories

this expression for $\overline{u_{g}^{\prime}(t) u_{g}^{\prime}(\eta)}$ into Equation (3.5) gives:

$$
\begin{aligned}
\overline{u_{g}^{\prime}(t) \overline{u_{p}(t)}} & =\frac{e^{\frac{-t}{\tau_{p}}}}{\tau_{p}} \int_{0}^{t} e^{\frac{\eta}{\tau_{p}}} \overline{u_{g}^{\prime 2}} e^{\frac{-(t-\eta)}{\tau^{*}}} d \eta+e^{\frac{-t}{\tau_{p}}} \overline{u_{g}^{\prime}(t) u_{p}(0)} \\
& =\frac{\overline{u_{g}^{\prime 2}}}{\tau_{p}}\left(\frac{\tau_{p} \tau^{*}}{\tau^{*}+\tau_{p}}\right)\left(e^{0}-e^{-t\left(\frac{1}{\tau_{p}}+\frac{1}{\tau^{*}}\right)}\right)+e^{\frac{-t}{\tau_{p}}} \overline{u_{g}^{\prime}(t) u_{p}(0)}
\end{aligned}
$$

The steady-state value for $\overline{u_{g}^{\prime} u_{p}^{\prime}}$ is therefore given by:

$$
\lim _{t \rightarrow \infty} \overline{u_{g}^{\prime} u_{p}^{\prime}}=\frac{\overline{u_{g}^{\prime 2}}}{\tau_{p}}\left(\frac{\tau_{p} \tau^{*}}{\tau^{*}+\tau_{p}}\right)=\overline{u_{g}^{\prime 2}}\left(\frac{\tau^{*}}{\tau^{*}+\tau_{p}}\right)
$$

Recall from Section 2.3.4 that the model of Mostafa of Mongia [30] was of this form. with the gas-particle correlation modeled as:

$$
\overline{u_{g}^{\prime} u_{p}^{\prime}}=\overline{u_{g}^{\prime 2}}\left(\frac{\tau_{L I}}{\tau_{L I}+\tau_{p}}\right)
$$

where the Lagrangian time scale $\tau_{L I}$ is given by $\tau_{L I}=0.35 \frac{k}{\epsilon}$.

Equation (3.9) does not account for the crossing trajectory effect. Recall that the crossing trajectory effect occurs when a particle traverses an eddy and enters another before the decay of the original eddy. This occurs due to free fall velocity of the particle. or in some cases, another strong body force acting on the particle. A result of the crossing trajectory effect is a reduction in the Lagrangian time scale, due to the decreased 
correlation of the fluid velocities that the particle 'sees' [39]. An effective turbulence modulation model should be capable of incorporating this effect.

\subsection{The Model of Lightstone and Hodgson}

The turbulence modulation model of Lightstone and Hodgson incorporates the crossing trajectory effect into the timescale $\tau^{*}$. This model follows from the work of Hennick and Lightstone [16] and Lightstone and Raithby [25].

As discussed in the previous section, the autocorrelation function, $R_{g p}$, is a function of the change in time and position over a timestep $\delta t$ and can be approximated by:

$$
R_{g p}(\delta t, \delta x)=e^{-\frac{\delta \epsilon}{\tau-}}
$$

where $\delta t=t-\eta$.

As a simplification the autocorrelation is decomposed into Lagrangian and Eulerian components:

$$
R_{g p}(\delta t, \delta x) \cong R_{\mathrm{LAG}}(\delta t) R_{E U L}(\delta x)
$$

As a particle travels through an eddy, the distance it travels can be approximated by the relationship $\delta x=u_{R E L} \delta t$, where $u_{R E L}$ is the velocity difference between the particle and the fluid. This allows the Eulerian correlation function to be related to the Lagrangian correlation function.

The Lagrangian correlation function is given by [18]:

$$
R_{L A G}(\delta t)=e^{\frac{-\delta t}{\tau L I}}
$$

It follows then, that the Eulerian correlation can be approximated by:

$$
R_{E U L}\left(u_{R E L} \delta t\right)=e^{\frac{-u_{R E L} \delta t}{L_{I}}}
$$

where $u_{R E L}=\left|\overrightarrow{u_{g}}-\overrightarrow{u_{p}}\right|$ and $L_{I}$ is the Lagrangian integral length scale.

Thus,

$$
\begin{aligned}
R_{g p} & \cong \exp \left(\frac{-\delta t}{\tau_{L I}}\right) \exp \left(\frac{-u_{R E L} \delta t}{L_{I}}\right) \\
& =\exp \left(-\delta t\left(\frac{1}{\tau_{L I}}+\frac{u_{R E L}}{L_{I}}\right)\right)
\end{aligned}
$$


Comparing this result with Equation (3.10) gives the following expression for $T^{*}$ :

$$
\frac{1}{\tau^{*}}=\frac{1}{\tau_{L I}}+\frac{u_{R E L}}{L_{I}}
$$

Now expressions for the Lagrangian integral length and time scales, $L_{I}$ and $\tau_{L I}$ respectively, are left to be derived.

Lightstone and Raithby [25] analytically derived an expression for $\tau_{L I}$. As discussed in Section 5.3, Taylor [42] showed that, for fluid particles

$$
\frac{1}{2} \frac{d \overline{x_{p}{ }^{2}}}{d t}=\overline{u_{p}^{2}} \tau_{L I}
$$

Csanady [10] defined turbulent viscosity, $\nu_{t}$ in terms of the particle dispersion such that

$$
\nu_{t}=\frac{1}{2} \frac{d \overline{x^{2}}}{d t}
$$

This expression applies to a turbulent Schmidt number of unity.

An expression for $\tau_{L I}$ is obtained by combining Equations (3.16) and (3.17) with the expression for turbulence viscosity from the $k-\epsilon$ model [21],

$$
\nu_{t}=C_{\mu} \frac{k^{2}}{\epsilon}
$$

where $C_{\mu}=0.09$.

This gives the following expression for $\tau_{L I}$ :

$$
\tau_{L I}=\frac{3}{2} C_{\mu} \frac{k}{\epsilon}=0.135 \frac{k}{\epsilon}
$$

An expression for $L_{l}$ is also obtained from analytical considerations [16]. Using Taylor's analysis [42] an expression for the eddy lifetime is obtained, such that

$$
t_{e}=2 \tau_{L I}
$$

The Lagrangian integral length scale will be the product of a velocity and the eddy lifetime. Taking the velocity to be $\sqrt{2 / 3 * k}$, the following expression for $L_{I}$ is obtained:

$$
L_{I}=2 \tau_{L I} \sqrt{\frac{2}{3} k}
$$

Incorporating all of the above components into the expression for the source term to 
the $k$-equation gives:

$$
\begin{aligned}
\overline{u_{g i}^{\prime} F_{p i}^{\prime}} & =\frac{\alpha}{\tau_{p}}\left(\overline{u_{g i}^{\prime} u_{g i}^{\prime}}-\overline{u_{g i}^{\prime} u_{p i}^{\prime}}\right) \\
& =\frac{\alpha}{\tau_{p}}\left(\overline{u_{g i}^{\prime} u_{g i}^{\prime}}-\overline{u_{g i}^{\prime} u_{g i}^{\prime}}\left(\frac{\tau^{*}}{\tau^{*}+\tau_{p}}\right)\right) \\
& =\frac{2 k \alpha}{\tau_{p}}\left(1-\frac{\tau^{*}}{\tau^{*}+\tau_{p}}\right)
\end{aligned}
$$

where:

$$
\begin{aligned}
\frac{1}{\tau^{*}} & =\frac{1}{\tau_{L I}}+\frac{u_{R E L}}{L_{I}} \\
\tau_{L I} & =0.135 \frac{k}{\epsilon} \\
L_{I} & =2 \tau_{L I} \sqrt{\frac{2}{3} k}
\end{aligned}
$$

An expression for the source term to the $\epsilon$ equation, $S_{\epsilon}$, is obtained by dividing Equation (3.22) by a timescale equal to $k / \epsilon$. This yields the following expression for the source to the $\epsilon$ equation:

$$
S_{\epsilon}=-\frac{1}{\rho_{g}} \frac{2 \epsilon \alpha}{\tau_{p}}\left(1-\frac{\tau^{*}}{\tau^{*}+\tau_{p}}\right)
$$

The behaviour of this model can be examined by considering the limiting cases of relative velocity and particle response time, $\tau_{p}$.

Consider first the case of fluid particles. In this limit there is no relative velocity and $u_{R E L}$ is zero. The Eulerian correlation goes to zero as required, and $\tau^{*}$ is equal to the Lagrangian interaction time, $\tau_{L I}$. Looking at the correlation $\overline{u_{g}^{\prime} u_{p}^{\prime}}$, for fluid particles $\tau_{p}$ is zero and as required the result $\overline{u_{g}^{\prime} u_{p}^{\prime}}=\overline{u_{g}^{\prime} u_{g}^{\prime}}$ is obtained. Conversely, very heavy particles are not able to follow the turbulence of the fluid. This results in a large relative velocity. between the particle and the fluid; the Eulerian correlation will dominate and $\boldsymbol{r}^{*}$ will approach $L_{I} / u_{R E L}$. Since the particles don't respond to the turbulence it is expected that $\overline{u_{g}^{\prime} u_{p}^{\prime}}$ will be equal to zero. Taking the limit of the expression for the gas-particle correlation as $\tau_{p}$ approaches infinity shows that this is indeed the case.

This model does not capture turbulence production (wake) effects. The gas-particle interaction described here is strictly due to velocity differences between the two phases (drag effects). 


\subsection{Summary}

This chapter introduced the turbulence modulation model of Lightstone and Hodgson. The exact expression for the correlation $\overline{u_{g}^{\prime} u_{p}^{\prime}}$ is derived in detail and the new model is presented. This model is similar to the model of Mostafa and Mongia [30], howerpr their model does not capture crossing trajectory effects. The model will only predict a reduction in turbulent kinetic energy. 


\section{Chapter 4}

\section{Numerical Solution}

\subsection{Introduction}

This chapter outlines the numerical methods used to solve the governing equations presented in Chapter 2. Discretization and evaluation of the equations for mass, momentim. $k$ and $\epsilon$ is discussed and an overview of the treatment of boundary conditions presented. The second half of the chapter discusses the numerical solution of the particulate phase including the implementation of the SSF model and the source terms to the momentum. $k$ and $\epsilon$ equations. It should be noted that much of the information in this chapter is taken from the TASCflow theory manual [2] and the reader is referred there for more information.

\subsection{Numerical Solution to the Governing Equations for the Gas-Phase}

The first half of this chapter will focus on the discretization and solution of the governing equations for the gas-phase. TASCflow uses a finite element based finite volume approach to solving the governing equations. This allows TASCllow to take advantage of the geometric flexibility of finite element methods while maintaining the physics of the flow through the use of the finite volume approach.

\subsubsection{Grid Specification}

TASCflow uses a finite element approach to control volume creation. A grid is supplied in terms of $x, y$ and $z$ locations and nodes are distributed throughout the computational domain. At each node the values for all dependent variable (pressure, velocity $k . \epsilon$ etc) are determined. The indices $i, j$, and $k$ refer to each node contained in the domain. 


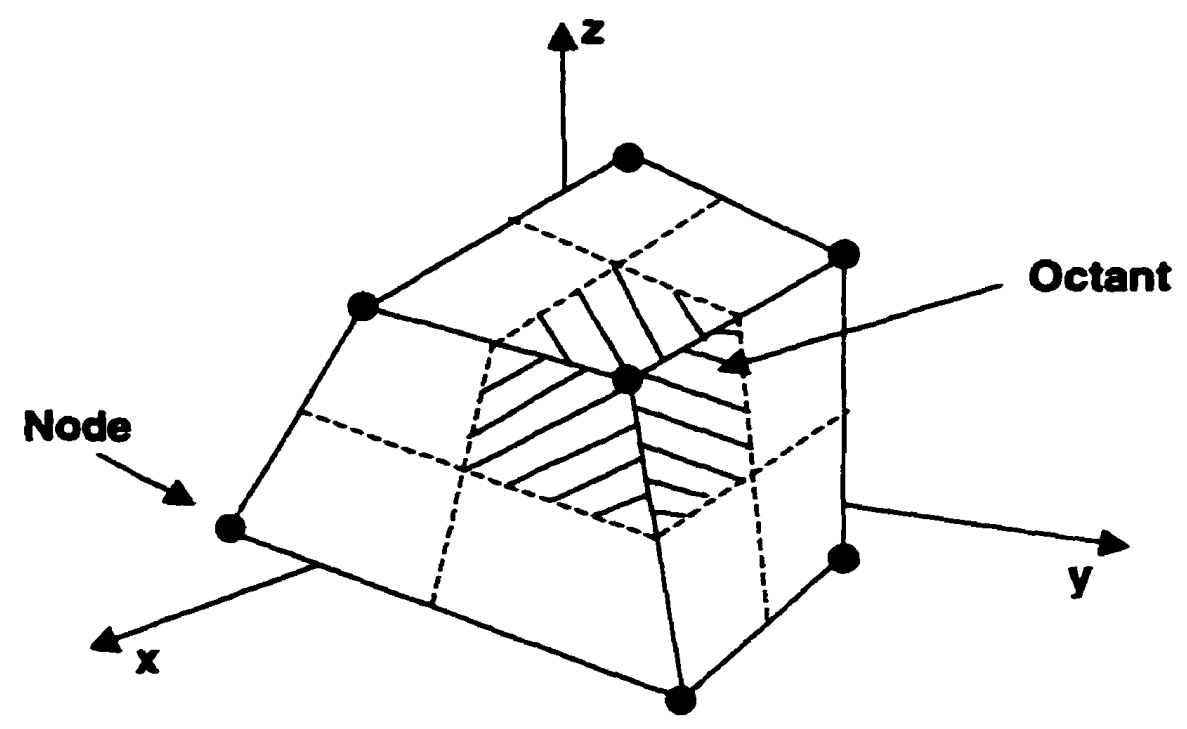

Figure 4.1: Flux element with 7 of the 8 nodes visible

A flux element is a linear, hexahedral element made up of eight nodes as shown in Figure 4.1. To create control volumes within the computational domain each flux element is divided into eight octants whose faces bisect the faces of the flux element. A control volume is then defined by each of the four octants which surround a node. Integration points are located at the mid-point of each of the interior faces. In 3-dimensions. a flux element is made up of 8 nodes, 8 octants and 12 integration points.

Boundary control volumes touch the boundary at one or more faces. In 3-dimensions boundary control volumes consist of 4 octants and 6 integration points. A 2-dimensional representation of a boundary and interior control volume is shown in Figure 4.2

\subsubsection{Mass and Momentum Equations}

The mean form of the governing equations for mass and momentum as presented in Section 2.3.3 are:

$$
\begin{aligned}
\frac{\partial \rho_{g}}{\partial t}+\frac{\partial \rho_{g} U_{g i}}{\partial x_{i}} & =0 \\
\rho_{g} \frac{\partial U_{g i}}{\partial t}+\rho_{g} U_{g j} \frac{\partial U_{g i}}{\partial x_{j}} & =-\frac{\partial \bar{P}}{\partial x_{i}}+\frac{\partial}{\partial x_{j}}\left(\mu_{\mathrm{eff}}\left(\frac{\partial U_{g i}}{\partial x_{j}}+\frac{\partial U_{g j}}{\partial x_{i}}\right)\right)+\overline{F_{i}}-\overline{F_{p i}}
\end{aligned}
$$

In keeping with the finite volume method, the above equations are integrated over a 


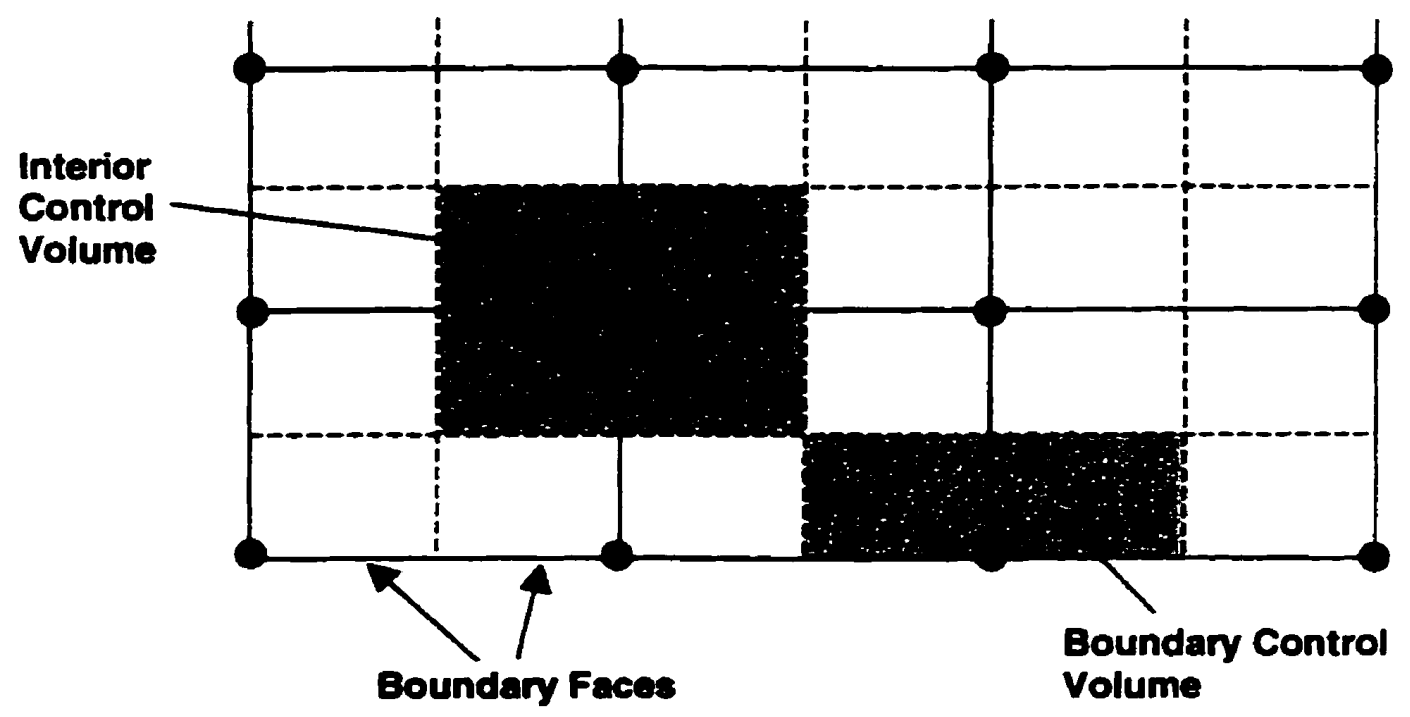

Figure 4.2: Boundary and Interior Control Volumes

fixed control volume. Using Gauss' theorem this results in:

$$
\begin{aligned}
\frac{\partial}{\partial t}\left(\int_{\mathfrak{F}} \rho d \digamma\right)+\int_{s} \rho U_{j} d n_{j}= & 0 \\
\frac{\partial}{\partial t}\left(\int_{\mathfrak{F}} \rho U_{i} d \digamma\right)+\int_{s} \rho U_{j} U_{i} d n_{j}= & -\int_{s} P d n_{i}+\int_{s} \mu_{\mathrm{eff}}\left(\frac{\partial U_{i}}{\partial x_{j}}+\frac{\partial U_{j}}{\partial x_{i}}\right) d n_{j} \\
& +\int_{\mathscr{F}} \overline{F_{i}} d \mathscr{W}-\int_{\mathfrak{F}} \overline{F_{p i}} d \digamma
\end{aligned}
$$

where $n_{j}$ is the outward normal surface vector and $\forall$ represents the volume.

These integral equations are evaluated at each control volume. The volume integrals are discretized across the control volume while the surface integrals are evaluated at the integration points. This gives rise to the following discrete form of the integral equations:

$$
\begin{aligned}
& \forall\left(\frac{\rho-\rho^{o}}{\Delta t}\right)+\sum_{i p}\left(\rho U_{j} \Delta n_{j}\right)_{i p}=0 \\
& \rho \nvdash\left(\frac{U_{i}-U_{i}^{o}}{\Delta t}\right)+\sum_{i p} \dot{m}_{i p}\left(U_{i}\right)_{i p}=\sum_{i p}\left(P \triangle n_{i}\right)_{i p} \\
&+\sum_{i p}\left(\mu_{\text {eff }}\left(\frac{\partial U_{g i}}{\partial x_{j}}+\frac{\partial U_{g j}}{\partial x_{i}}\right) \Delta n_{j}\right)_{i p}+\overline{F_{i}} \nvdash-\overline{F_{p i}} \nvdash
\end{aligned}
$$

where $m_{i p}=\left(\rho u_{g j} \Delta n_{j}\right)_{i p}^{o}$ and the superscript $o$ refers to value at the previous time 
step. The transient term, $\forall\left(\frac{\rho-\rho^{\circ}}{\Delta t}\right)$, is approximated by a first-order Euler method.

\subsection{3 $k$ and $\epsilon$ Equations}

Recall from Section 2.3.3 the modeled forms of the equations for $k$ and $\epsilon$ :

$$
\begin{aligned}
& \frac{D k}{D t}=\frac{1}{\rho} \frac{\partial}{\partial x_{k}}\left[\frac{\mu_{t}}{\sigma_{k}} \frac{\partial k}{\partial x_{k}}\right]+\frac{\mu_{t}}{\rho}\left(\frac{\partial U_{i}}{\partial x_{k}}+\frac{\partial U_{k}}{\partial x_{i}}\right) \frac{\partial U_{i}}{\partial x_{k}}-\epsilon-S_{k} \\
& \frac{D \epsilon}{D t}=\frac{1}{\rho} \frac{\partial}{\partial x_{k}}\left[\frac{\mu_{t}}{\sigma_{\epsilon}} \frac{\partial \epsilon}{\partial x_{k}}\right]+\frac{C_{1} \mu_{t}}{\rho} \frac{\epsilon}{k}\left(\frac{\partial U_{i}}{\partial x_{k}}+\frac{\partial U_{k}}{\partial x_{i}}\right) \frac{\partial U_{i}}{\partial x_{k}}-C_{2} \frac{\epsilon^{2}}{k}+S_{\epsilon}
\end{aligned}
$$

The discrete forms to these equations are obtained in the same manner as any scalar transport equation. The equations can be represented in a simplified form as:

$$
\rho \frac{\partial \phi}{\partial t}+\rho U_{g k} \frac{\partial \phi}{\partial x_{k}}=\frac{\partial}{\partial x_{k}}\left[\frac{\mu_{t}}{\sigma_{k, \epsilon}} \frac{\partial \phi}{\partial x_{k}}\right]+S^{\phi}
$$

where $\phi$ represents either $k$ or $\epsilon$ and $S^{\phi}$ represents all of the sources to the $k$ and $\epsilon$ equations including the effects of the particles.

Integrating over a fixed control volume and applying Gauss' theorem yields:

$$
\frac{\partial}{\partial t}\left(\int_{\leftarrow} \rho \phi d \nvdash\right)+\int_{s} \rho U_{j} \phi d n_{j}=\int_{s} \frac{\mu_{t}}{\sigma_{k, \varepsilon}} \frac{\partial \phi}{\partial x_{k}} d n_{j}+\int_{\mathfrak{F}} S^{\circ} d \digamma
$$

This equation is discretized in the same way as the mass and momentum equations.

$$
\rho F\left(\frac{\phi_{N}-\phi_{N}^{o}}{\Delta t}\right)+\sum_{i p} \dot{m}_{i p} \phi_{i p}=\sum_{i p}\left(\frac{\mu_{t}}{\sigma_{k, \epsilon}} \frac{\partial \phi}{\partial x_{k}} \Delta n_{j}\right)_{i p}+S^{\phi} F
$$

The source term to the $k$ equation is given by:

$$
S^{k}=\mu_{t}\left(\frac{\partial U_{i}}{\partial x_{k}}+\frac{\partial U_{k}}{\partial x_{i}}\right) \frac{\partial U_{i}}{\partial x_{k}}-\rho \epsilon-\rho S_{k}
$$

Integrating over a control volume gives:

$$
\int_{F} S^{k} d F \approx \Delta F\left[\mu_{t}\left(\frac{\partial U_{i}}{\partial x_{k}}+\frac{\partial U_{k}}{\partial x_{i}}\right) \frac{\partial U_{i}}{\partial x_{k}}-\rho \epsilon-\rho S_{k}\right]
$$

The source term to the $\epsilon$ equation is given by

$$
S^{\epsilon}=C_{1} \mu_{t} \frac{\epsilon}{k}\left(\frac{\partial U_{i}}{\partial x_{k}}+\frac{\partial U_{k}}{\partial x_{i}}\right) \frac{\partial U_{i}}{\partial x_{k}}-C_{2} \rho \frac{\epsilon^{2}}{k}+\rho S_{\epsilon}
$$


Integrating over a control volume gives,

$$
\int_{\forall} S^{\epsilon} d \forall \approx \Delta V\left[\frac{\epsilon}{k}\left(C_{1} \mu_{t}\left(\frac{\partial U_{i}}{\partial x_{k}}+\frac{\partial U_{k}}{\partial x_{i}}\right) \frac{\partial U_{i}}{\partial x_{k}}-C_{2} \rho \epsilon\right)+\rho S_{\epsilon}\right]
$$

The non-linearity in this equation needs to be dealt with. Raithby [2] suggests that it is important to linearize the equation such that the turbulent time scale. $\frac{k}{\epsilon}$. remains constant. This yields,

$$
\int_{F^{\prime}} S^{\epsilon} d F \approx \Delta \Psi\left[\left(\frac{\epsilon}{k}\right)^{o}\left(C_{\mathrm{L}} \mu_{t}\left(\frac{\partial U_{i}}{\partial x_{k}}+\frac{\partial U_{k}}{\partial x_{i}}\right) \frac{\partial U_{i}}{\partial x_{k}}-C_{2} \rho \epsilon\right)+\rho S_{\epsilon}\right]
$$

where the superscript $o$ indicates that the value is taken from the previous time step.

\subsubsection{Evaluation of the Discretized Equations}

A finite element approach is used to evaluate the diffusion terms. This approach utilizes shape functions, $N_{n}$, to evaluate the derivatives for the diffusion terms.

$$
\left.\frac{\partial \phi_{i p}}{\partial x}\right|_{i p}=\left.\sum_{n} \frac{\partial N_{n}}{\partial x}\right|_{i p} \phi_{n}
$$

The Cartesian derivatives of the shape functions are transformed into their local derivatives using a Jacobian transformation matrix. These local shape function derivatives are evaluated at the location where each integration point face intersects the element edge. The resulting 'linear-linear' evaluations of the Cartesian derivatives are formally' first-order accurate.

The discretized pressure term is given by,

$$
\sum_{i p}\left(P \triangle n_{i}\right)_{i p}
$$

These terms are evaluated at each integration point using the finite element approach. Once again the local shape functions, $N_{n}$, are used so that,

$$
P_{i p}=\sum_{n} N_{n} P_{n}
$$

The shape functions are evaluated using linear-linear interpolation.

Evaluation of the advection terms is achieved through a finite volume approach. TASCflow has a variety of schemes available to evaluate the advection terms. These schemes include the upwind difference scheme, linear profile and mass weighted skewed upstream difference schemes which can include physical advection correction terms. 


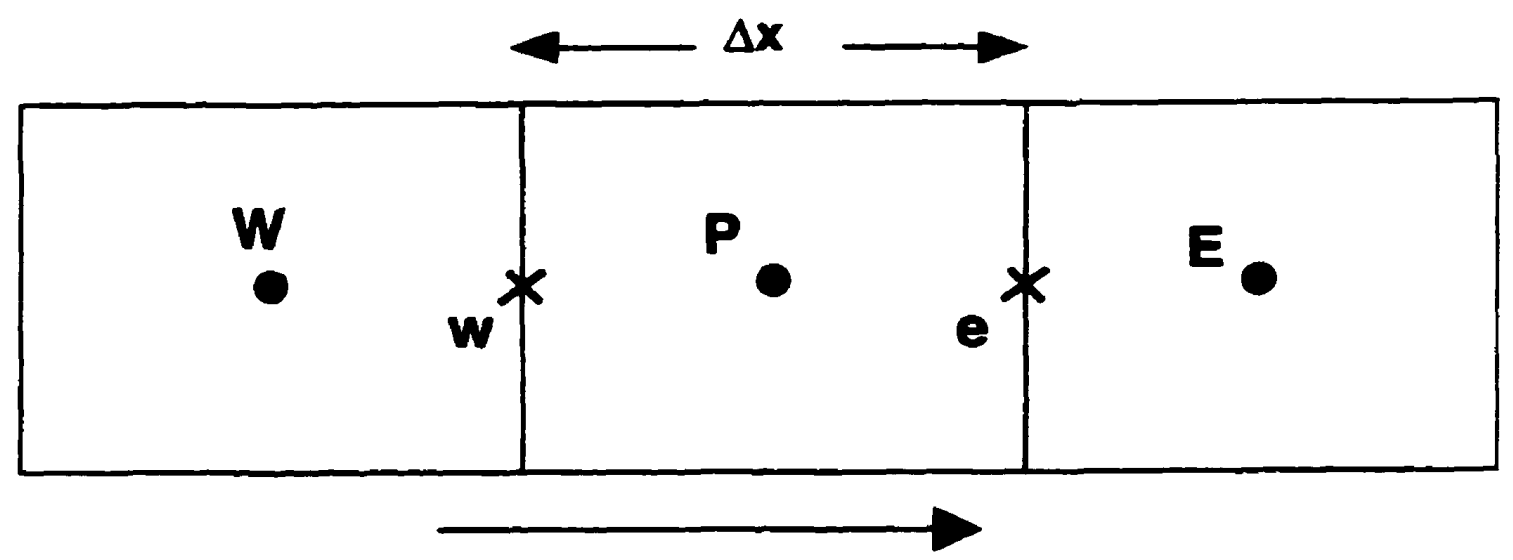

Figure 4.3: One-Dimensional flow moving from point $P$ to $E$

The starting point to these differencing schemes lies in the upwind difference scheme (UDS) and the central difference scheme (CDS). Consider a one-dimensional flow moring from node $P$ to node $E$ as shown in Figure 4.3. The UDS very simply applies a value of $\phi$ to the integration point $e$ that is equal to the value of $\phi$ at the node immediately upstream such that,

$$
\phi_{e}=\phi_{P}
$$

This assumption is reasonable for high-speed flows, however it neglects the influence of the downstream node $\mathrm{E}$. For low-speed or skewed flows node $\mathrm{E}$ will significantly affect the point $e$. The central difference scheme attempts to capture this effect by introducing a correction, $\Delta \phi_{e}$, to the UDS. This correction is approximated by,

$$
\triangle \phi_{e} \approx \frac{\Delta x}{2}\left(\frac{\partial \phi}{\partial x}\right)_{e}
$$

Using a finite difference discretization the correction term becomes,

$$
\triangle \phi_{e} \approx \frac{1}{2}\left(\phi_{E}-\phi_{P}\right)
$$

and an estimate for $\phi_{e}$ is given by,

$$
\phi_{e}=\frac{1}{2} \phi_{P}+\frac{1}{2} \phi_{E}
$$

Now the value at the integration point is equally influenced by nodes $P$ and $E$. This is obviously not a physical result either.

An alternative approach to the CDS is the physical advection correction scheme 
(PAC). The PAC scheme attempts to apply a correction, $\triangle \dot{\phi}_{e}$, to the UDS which properlyreflects the physical interactions of the advection, diffusion and source terms.

Consider an advection dominated transport equation in non-conservative form:

$$
\rho_{g} U_{g} \frac{\partial \phi}{\partial x}+\rho_{g} V_{g} \frac{\partial \phi}{\partial y}+\rho_{g} W_{g} \frac{\partial \phi}{\partial z}=\dot{S}^{\phi}
$$

Rearranging yields;

$$
\frac{\partial \phi}{\partial x}=\frac{1}{\rho_{g} U_{g}}\left[\dot{S}^{\phi}-\rho_{g} V_{g} \frac{\partial \phi}{\partial y}-\rho_{g} W_{g} \frac{\partial \phi}{\partial z}\right]
$$

Substituting this result into the approximation for the correction term given in $\mathrm{Eq}_{\mathrm{q}}$. (4.20) gives the following expression for the correction term:

$$
\Delta \phi_{e} \approx \frac{\Delta x}{2 \rho_{g} U_{g}}[\dot{S}^{\phi}-\underbrace{\rho_{g} V_{g} \frac{\partial \phi}{\partial y}}_{I}-\underbrace{\rho_{g} W_{g} \frac{\partial \phi}{\partial z}}_{I I}]
$$

Now all that remains is the evaluation of terms I and II. Discretization of these terms can lead to non-physical wiggles. This can be overcome through the use of the Skew Upstream Difference (SUD) Scheme [34] which removes the advective component of the PAC term. This is achieved through the evaluation of the advection equation along a stream-line:

$$
\rho_{g} U_{g} \frac{\partial \dot{\varphi}}{\partial x}+\rho_{g} V_{g} \frac{\partial \phi}{\partial y}+\rho_{g} W_{g} \frac{\partial \phi}{\partial z}=\rho_{g} \overrightarrow{V_{g}} \frac{\partial \dot{\phi}}{\partial s}
$$

where $\vec{V}_{g}$ is the velocity along the streamline and $s$ is the distance in the streamwise direction. This value of $\phi$ at the integration point $e$ is now determined by,

$$
\phi_{e} \approx \phi_{u}+\Delta \phi_{e}
$$

where $u$ us a point on the streamline upstream from $e$. The correction factor $\Delta o_{e}$ is approximated by

$$
\Delta \dot{\phi}_{e} \approx l \frac{\partial \phi}{\partial s}
$$

where $l$ is the distance along the streamline between $e$ and $u$.

Two schemes are available in TASCflow to evaluate the value of $\phi_{u}$ : the Linear Profile (LP) scheme and the Mass Weighted (MW) scheme [34, 26, 19, 35, 37. 38] The LP scheme uses linear interpolation of nodal values to determine $\phi_{u}$. This approach is formally second-order with only a small possibility of non-physical wiggles. In the MII scheme $\phi_{u}$ is evaluated by performing a weighted average of the nodes surrounding $u$. The weighting factors are based on the mass flows passing through the control volume faces 
surrounding point $u$. This scheme is only first-order accurate, but completely eliminates the possibility of non-physical wiggles occurring in the solution.

The simulations performed for this research use the upwind difference scheme without physical advection correction terms to evaluate the advection terms. These are the default values in TASCflow.

\subsubsection{Boundary Conditions}

Boundary conditions are specified by the user for each boundary face until all boundary control volume equations are closed. The boundary condition information must be sufficient to form a well-behaved system of equations whose solution is possible. The simulations performed in this work uses four types of boundary conditions: inlet. outlet. symmetry and no-slip wall.

\section{No-Slip Wall}

When viscous flow comes into contact with a wall boundary the fluid is stationary, relative to the wall, at the point of contact. That is to say, that at the wall, the fluid relocity normal to the wall is zero. Therefore there is no mass flowing through the wall and at each boundary integration point the following condition is imposed:

$$
\dot{m}_{i p}=0
$$

In turbulent flows there are very large gradients near the wall. In order to resolve the solution near the wall many nodes are required in this region. Since this is very costly the logarithmic law of the wall is used to provide boundary conditions for the near-wall region, defined as the region between the wall and the first interior node (see Figure 4.4). The log-law of the wall relates the near-wall tangential velocity to the wall shear stress through the following relationships:

$$
\begin{aligned}
& u^{+}=\frac{1}{0.41} \ln \left(y^{+}\right)+C \\
& y^{+}=\frac{\Delta n \sqrt{\rho \tau_{w}}}{\mu} \\
& u^{+}=\frac{U}{u_{\tau}} \\
& u_{\tau}=\sqrt{\frac{\tau_{w}}{\rho}}
\end{aligned}
$$




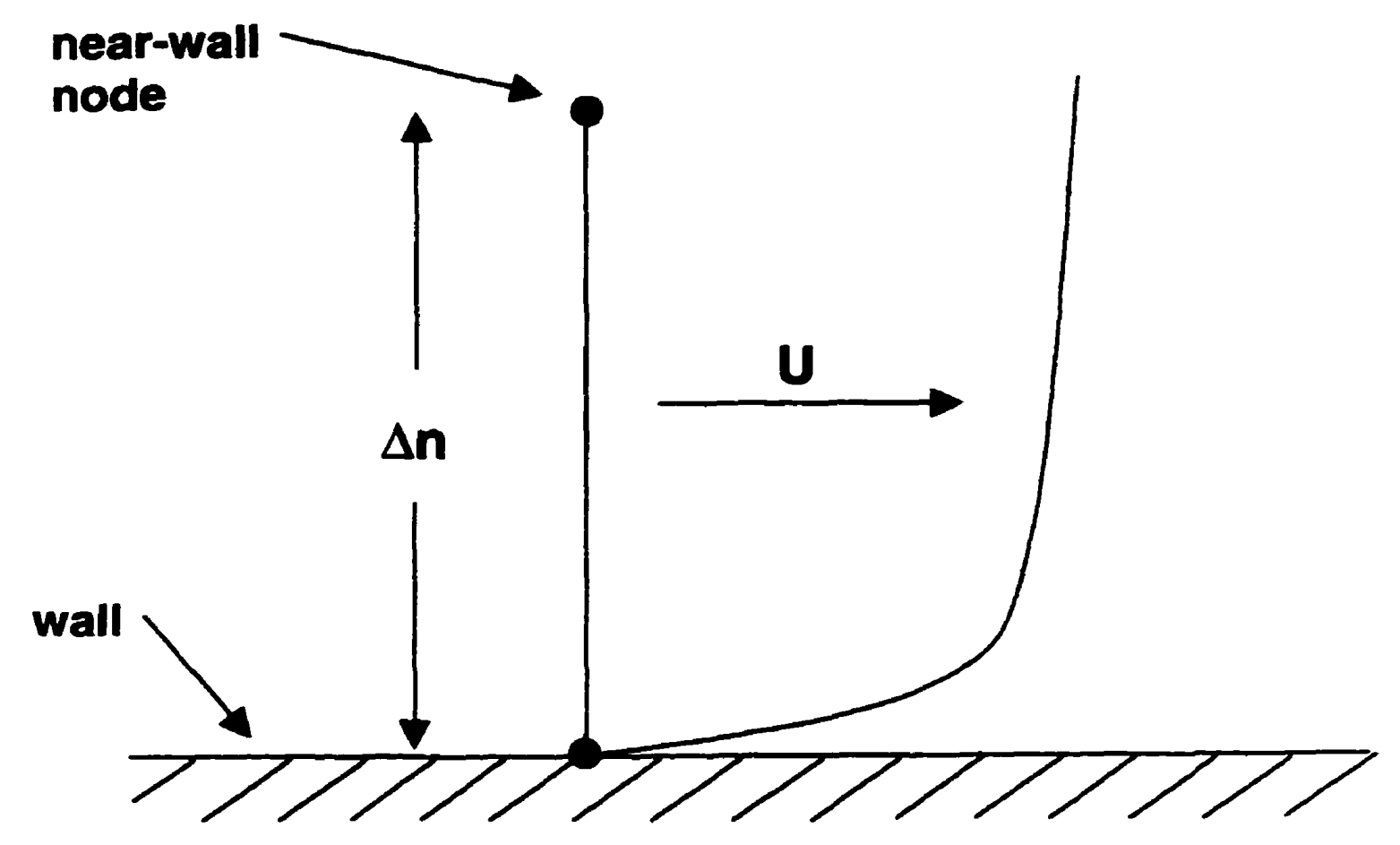

Figure 4.4: Near-Wall Region

$$
\frac{\tau_{w}}{\rho}=k \sqrt{C_{\mu}}
$$

$\Delta n$ is the distance from the node nearest the wall to the wall and $C$ is a constant which can take on values from 4.9 to 5.5 .

In order to solve the $k$ and $\epsilon$ in the near-wall region the boundary conditions imposed must be consistent with the log-law of the wall. This is achieved by making several assumptions as to the nature of the production and dissipation of turbulent energy in the near-wall region: local equilibrium exists between production and dissipation. the wall shear stress is constant over the near-wall region, and production of $k, P_{k}$. is a function of $u^{+}, y^{+}, U$ and $\Delta n$ [2].

\section{Symmetry Condition}

The symmetry boundary condition is used in situations where the flow on one side of a plane is a mirror image of the flow on the opposite side of the plane. The fluid relocity normal to the symmetry plane is zero. This leads to the boundary condition that at each boundary integration point lying on a symmetry plane the mass flow is also zero. The momentum equation is evaluated at the symmetry plane by assuming that all components 
of the viscous shear stress tangent to the symmetry plane are zero. The $k$ and $\epsilon$ equations are resolved at the symmetry boundary by imposing the condition that the gradients of $k$ and $\epsilon$ normal to the wall is zero.

\section{Outlet Condition}

The mass flow and velocity at an outlet boundary are determined as an implicit result of the flow simulation. Pressure can be specified in several different ways; over the entire outlet region, at one location in the outlet, as an average pressure over the surface or left unspecified at the outlet (but specified at some location within the computational domain). In the current investigation the outlet pressure is specified at one location. a single control volume face.

\section{Inlet Condition}

There are several ways of specifying an inlet boundary condition: velocity, mass flow: static pressure and velocity or pressure. This investigation uses a velocity specified boundary. The user specified velocity is used to calculate the mass flow through the inlet face, such that:

$$
\dot{m}_{i p}=\left.\rho_{i p} A_{i}\right|_{i p} u_{i s p e c}
$$

where $A_{i}$ is the boundary area and $u_{i s p e c}$ is the specified velocity.

The inlet momentum flow, $F^{\text {inlet }}$ is the sum of advection, pressure and viscous force components,

$$
F^{\text {inlet }}=F^{\text {advect }}+F^{\text {press }}+F^{\text {visc }}
$$

For a velocity specified boundary the advection force is calculated as the product of the mass flow and specified velocity. The pressure force is based on interpolation of the control volume pressures and viscous forces are computed using the control volume velocities and the specified velocity.

To properly resolve the boundary solution for the $k$ and $\epsilon$ equations, the inlet turbulence quantities must be specified. These quantities are specified in terms of turbulence intensity, $T_{i}$, and eddy length scale, $L_{\epsilon}$ which are given by:

$$
\begin{aligned}
& T_{i}=\frac{\sqrt{\frac{2}{3} k}}{|\vec{U}|} \\
& L_{\epsilon}=\frac{k^{3 / 2}}{\epsilon}
\end{aligned}
$$




\subsubsection{Pressure-Velocity Coupling}

A difficulty encountered in solving the governing equations is the decoupling between pressure and velocity. Referring again to Figure 4.3, when writing the discretized momentum equation for $U_{P}$, the velocity at node $\mathrm{P}$, the pressure gradient is discretized using a central difference formulation as follows:

$$
\begin{aligned}
\frac{\partial P_{P}}{\partial x} & =\frac{P_{e}-P_{w}}{\Delta x} \\
& =\frac{1 / 2\left(P_{P}+P_{E}\right)-1 / 2\left(P_{W}+P_{P}\right)}{\Delta x} \\
& =\frac{P_{E}-P_{W}}{2 \Delta x}
\end{aligned}
$$

So the pressure at node $\mathrm{P}, P_{P}$, does not appear in the momentum equation for $C_{P}$. The mass and momentum equations can therefore accept an oscillating pressure field as being smooth, i.e. $P_{i-1}=-10, P_{i}=+10, P_{i+1}=-10$ could be accepted, and the solution can become uncoupled.

One approach to overcoming this problem is to use a staggered grid method. This approach involves defining two staggered grids; on one grid pressure will be solved and on the other velocity [32]. While this method does effectively eliminate the decoupling problem, it is not ideal. Additional control volume accounting is required, and boundary conditions are difficult to impose.

An alternate approach is the method of colocated variables. To ensure that decoupling does not occur, the integration point velocities are directly related to the node-to-node pressure differences. This involves separating the velocity into two components. one dependent on the pressure gradient, $\hat{u}$, and one which is not, $u$. The mass flux is calculated at a control volume face using $\hat{u}$, while $u$ is used to calculate the momentum flux. For a detailed explanation of the colocation scheme see the TASCflow theory manual.

\subsubsection{Linear Solver}

The linear equation solver used in TASCflow uses an additive correction multigrid method to solve the coupled algebraic equations. Linear solvers are very effective at removing high-frequency errors but often convergence stalls on the remaining low-frequency error. In order to overcome this difficulty a multigrid technique is used. A fine grid is used to remove high-frequency error and the low-frequency error is passed down to a coarser grid where it 'appears' as high-frequency error.

The multigrid solver is used in a fixed W-cycle process. In this process the solver 
works at the coarser grid levels passing the solution up and down before going back to finer grid levels. In this way most of the error is eliminated at the coarse grid level. where it is least expensive, and only the high frequency error is left for the fine grid (which linear solvers are very effective at removing).

\subsection{Numerical Solution of the Particulate-Phase}

The last half of this chapter discusses the method by which the SSF model for particle dispersion is implemented and how the source term to the momentum and $k$ and $\epsilon$ equations are evaluated. The procedure developed in this research for implementing the source terms is also discussed.

\subsubsection{Implementation of the SSF Model}

In TASCflow the particle trajectories are determined according to the SSF model of Shuen, Chen and Faeth [40] discussed in Section 2.3.2.

Implementation of Lagrangian tracking in TASCflow involves the tracking of individual particles from their injection point until they escape the computational domain or some integration limit is met.

The starting point to Lagrangian tracking is to calculate the particle displacement. This is achieved using forward Euler integration of the particle velocity over the computational time step, $\delta t$.

$$
x_{i}^{n}=x_{i}^{o}+\frac{d x_{i}^{o}}{d t} \delta t
$$

The superscripts $n$ and 0 refer to new and old values respectively, $\delta t$ represents the computational time step (not to be confused with particle interaction time) and $\frac{d x_{i}^{o}}{d t}=u_{m}$. the particle velocity. At the end of the computational time step the new particle relocity is calculated according to the analytical solution to the particle equation of motion. Eq.(2.7). Assuming that gravity is the only body force acting on the particle and that the flow is within Stokes regime the solution is given by:

$$
u_{p}=u_{p}^{o} \exp \left(-\frac{\delta t}{\tau_{p}}\right)+\left(u_{g}-g \tau_{p}\right)\left(1-\exp \left(-\frac{\delta t}{\tau_{p}}\right)\right)
$$

As the particle travels through the control volumes its influence on the flow field is calculated as sources to the momentum, $k$ and $\epsilon$ equations.

Calculation of particle trajectories is performed in local coordinates using shape functions, $N_{n}$, which forces the path of the particles to be limited by the flux element bound- 
aries. This ensures the calculation of particle sources to the gas-phase for a single flux element and allows an adjoining element to be checked before the particle enters it. i.e in the case of a boundary.

The computational time step that may be taken in one step is limited by the following three criteria:

1. The particle cannot travel beyond an octant or flux element boundary in any time step.

2. The particle can only travel some user-specified fraction of the characteristic flux element in any step.

3. The time step is limited by the eddy lifetime and particle transit time.

In keeping with the SSF model of Shuen, Chen and Faeth, the gas-phase velocit! fluctuation remains constant over the particle/eddy interaction time. A new relocity fluctuation is determined from the randomly sample distribution only when the sum total of the computational timesteps is equal to the particle interaction time.

\subsubsection{Particle Injection}

In TASCflow particles are injected in groups. A group is defined as a set of particles with a given mass flow, $\dot{m}_{t}$, velocity, size and injection region. In order to obtain a statistically significant sample of particles, on the order of 1000 particles need to be injected per group [2]. In the current investigation, between 750 and 1500 particles are injected for each group.

Particles can be injected from a point, line, surface or volume. If the particles are injected from a point, then each particle will be injected from a single node. Each particle will be injected with a mass flow equal to the total mass flow for the group divided by the number of particles. If the particles are injected from a line, surface or volume then the number of particles injected in each control volume is determined such that the mass flow is constant for each segment or volume in the total injection region.

The number of particles injected in each region is determined in the following manner. The total area of the injection region, $A_{t}$, and the area of each segment of the injection region $i, A_{i}$, are calculated. The number of particles injected in each region. $n_{i}$. is determined by

$$
n_{i}=\max \left(1, n_{t} \frac{A_{i}}{A_{\ell}}\right)
$$

where $n_{t}$ is the total number of particles injected. 
The mass flow carried by each particle is also a function of the ratio of the segment areas and the total area. All particles in a given region have the same mass flux but in order to ensure that mass flow is constant across the total injection region the mass flow varies from region to region. Thus, the mass carried by particle, $j$, in segment $i$. is calculated from,

$$
\dot{m}_{j}=\frac{\dot{m}_{t}}{n_{i}} \frac{A_{i}}{A_{t}}
$$

where $\dot{m}_{t}$ is the total mass flow of the group.

\subsection{Implementation of Source Terms}

The effect of the particles on the gas-phase momentum are calculated as a source term to the momentum equation as discussed in Section 2.2.5. Recall that the source term has the form,

$$
F_{p}=\frac{\alpha}{\tau_{p}}\left(u_{g}-u_{p}\right)
$$

where $\alpha$ is the concentration of the particles and $\tau_{p}$ is the aerodynamic response time. The particle velocity $u_{p}(t)$ is known from the analytical solution to the particle equation of motion, Eq.(2.7):

$$
u_{p}(t)=u_{p}^{o} \exp \left(-\frac{t}{\tau_{p}}\right)+\left(u_{g}-g \tau_{p}\right)\left(1-\exp \left(-\frac{t}{\tau_{p}}\right)\right)
$$

Substituting Equation (4.40) into Equation (4.39) yields:

$$
F_{p}(t)=\frac{\alpha}{\tau_{p}}\left[u_{g}-u_{p}^{o} \exp \left(-\frac{t}{\tau_{p}}\right)-\left(u_{g}-g \tau_{p}\right)\left(1-\exp \left(-\frac{t}{\tau_{p}}\right)\right)\right]
$$

Rearranging Equation (4.41) gives,

$$
F_{p}(t)=\frac{\alpha}{\tau_{p}}\left[\left(u_{g}-u_{p}^{o}\right) \exp \left(-\frac{t}{\tau_{p}}\right)+g \tau_{p}\left(1-\exp \left(-\frac{t}{\tau_{p}}\right)\right)\right]
$$

The source to the momentum equation is obtained by averaging Equation (4.42) orer the computational time step, $\delta t$, such that:

$$
\begin{aligned}
-\overline{F_{p}(t)} & =\frac{1}{\delta t} \int_{0}^{\delta t}-F_{p}(t) d t \\
& =\frac{1}{\delta t} \int_{0}^{\delta t} \frac{\alpha}{\tau_{p}}\left[\left(u_{p}^{o}-u_{g}\right) \exp \left(-\frac{t}{\tau_{p}}\right)-g \tau_{p}\left(1-\exp \left(-\frac{t}{\tau_{p}}\right)\right)\right] d t
\end{aligned}
$$




$$
=\underbrace{\frac{\alpha}{\delta t}\left(u_{p}^{o}-u_{g}\right)\left(1-\exp \left(-\frac{\delta t}{\tau_{p}}\right)\right)}_{I}-\underbrace{\left[\alpha g+\alpha g \frac{\tau_{p}}{\delta t}\left(\exp \left(-\frac{\delta t}{\tau_{p}}\right)-1\right)\right]}_{I I}
$$

Term I represents the effect of the velocity difference between the gas and particle phases on the mean momentum while Term II is the effect of the interaction of the particle and gravity on the mean momentum. It is shown in Appendix $B$ that for $\delta t / \tau_{p} \ll 1$. Term II is negligible. This reduces the source term to:

$$
-\overline{F_{p}}=\frac{\alpha}{\delta t}\left(u_{p}^{o}-u_{g}\right)\left(1-\exp \left(-\frac{\delta t}{\tau_{p}}\right)\right)
$$

In TASCflow the particle source terms to the momentum equation are recalculated each time particles are injected. The momentum source terms are retained in memory and applied each time the fluid coefficients are calculated. The flow field adjusts for the presence of the particles and then the particles are injected again. After each injection they will follow a slightly different path through the new flow field. This cycle is repeated until the solution converges, that is to say, the average path that the particles travel through the flow field ceases to change from injection to injection.

The source terms to the $k$ and $\epsilon$ equations which account for turbulence modulation are implemented through a user modifiable source code available with TASCflow. The source terms are added to the $k$ and $\epsilon$ equations through subroutines that are called in the user modifiable source code.

The procedure developed in this research for implementing the source terms to the momentum, $k$ and $\epsilon$ equations is as follows:

1. The 'clean' flow field, that is the gas-phase without any sources, is solved.

2. The particles are injected and source terms to the momentum equations and particle concentration array calculated.

3. The gas-phase velocity and pressure solution is obtained with the momentum source terms held constant.

4. Momentum sources and particle concentration array are written out.

5. Solver is run again with the user modifiable source code. Here momentum sources and the particle concentration array are read in and applied to the momentum equations and source terms to the $k$ and $\epsilon$ equations are calculated (based on particle concentration array) and applied to the turbulence model. 
The procedure is repeated from step (2) until the solutions obtained at the end of step (5) cease to change (global convergence is reached). For the current investigation. convergence was reached after 3 global iterations (step (2) to step (5)) were performed.

\subsection{Summary}

This chapter outlined the numerical solution to a problem involving a gas-particle flow with turbulence modulation. The discretization of the governing equations was discussed and their implementation outlined. The SSF model used in TASCHow was presented and its implementation discussed. Also outlined was the evaluation and implementation of the source terms to the momentum, $k$ and $\epsilon$ equations. 


\section{Chapter 5}

\section{Validation}

Before attempting to implement the turbulence modulation models into TASCflow rigorous validation was performed. The motivation for the validation is two-fold: To make sure the code works as it should, and to get a measure of the accuracy of the predictions for flows which are less complex than those which exhibit turbulence modulation.

As a starting point a simple laminar pipe flow solution was obtained and compared against the analytical solution. Then a turbulent pipe flow simulation was performed and the solution was compared with experimental data and the analytical solution. Analysis was performed on the turbulent simulation to ensure grid-independence and to check the code's sensitivity to prescribed inlet condition, node distribution and log-law of the wall constants. Next, to verify the Lagrangian tracking portion of the code, experimental data of particle dispersion in a pipe was compared to the code's prediction. To verify that sources to the $k$ and $\epsilon$ equations were properly implemented through the userspecified subroutine, a grid-turbulence simulation was performed and the result compared to an analytical solution. Finally, the implementation of the sources to the momentum equations was verified analytically for a gas-particle laminar pipe flow.

\subsection{Laminar Pipe Flow}

The motivation behind validating a simple laminar pipe flow was to ensure that the procedure for using TASCflow was understood and that the computational grid used throughout this investigation was accepted by the code. In a pipe flow, fully-developed flow characteristics are independent of the $\theta$-direction and are functions of axial and radial positions only. It is useful to take advantage of this symmetry and model the circular pipe flow using a wedge shaped grid with perfectly reflecting boundaries on each radial wall. This effectively turns the three-dimensional problem into one of two-dimensions. resulting in a significant reduction in the number of nodes used in the grid. 


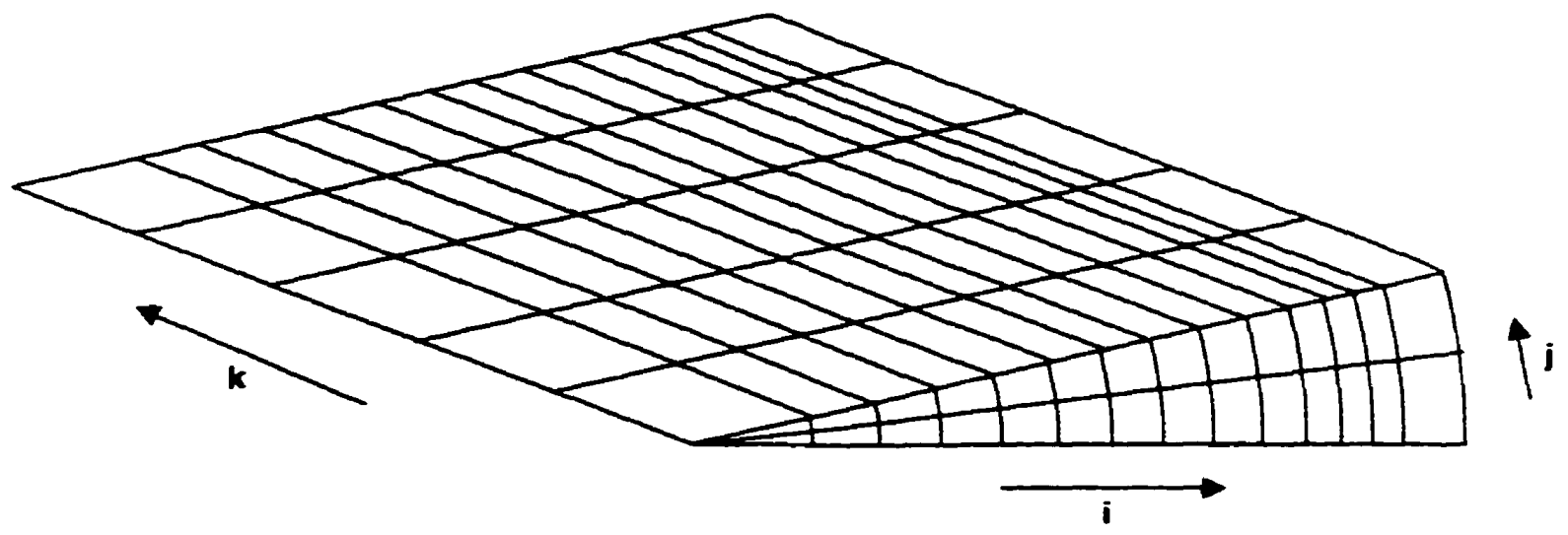

Figure 5.1: Computational Grid (truncated in k-direction)

The wedge-shape grid used in this investigation is shown in Figure 5.1. The wedge has an internal angle of $15^{\circ}$. Both of the radial walls and the collapsed point rere assigned perfectly reflecting symmetry boundary conditions. The curved wall was assigned at noslip wall boundary condition. The grid had 15 nodes in the $i$-direction, 3 nodes in the $j$-direction, and 1000 nodes in the $k$-direction. The nodes in the radial (i) direction were spaced closer together towards the wall where gradients are highest. The grid was designed to model the experiment of Tsuji et al [44] which will be discussed in detail in a later chapter. The pipe used in the experiments had a radius of $15.25 \mathrm{~mm}$ and a length of $5.11 \mathrm{~m}$. This is reflected in the geometry of the grid.

The analytical solution for axial velocity for laminar flow in a pipe is given br:

$$
u(r)=-\frac{d P}{d l} \frac{1}{4 \mu}\left(R^{2}-r^{2}\right)
$$

where $P$ is the pressure, $l$ is the pipe length, $\mu$ is the dynamic viscosity and $R$ is the radius of the pipe. The pressure change over the length of the pipe is given by:

$$
\frac{d P}{d l}=\frac{8 U \mu}{R^{2}}
$$

where $U$ is mean velocity in the axial direction. The results from the laminar simulation $(R e=100)$ are shown in Figure 5.2. The radial velocity profile predicted by the cocle is in excellent agreement with the analytical solution. It can therefore be concluded that the wedge-shaped grid can effectively model laminar flow through a circular pipe. 


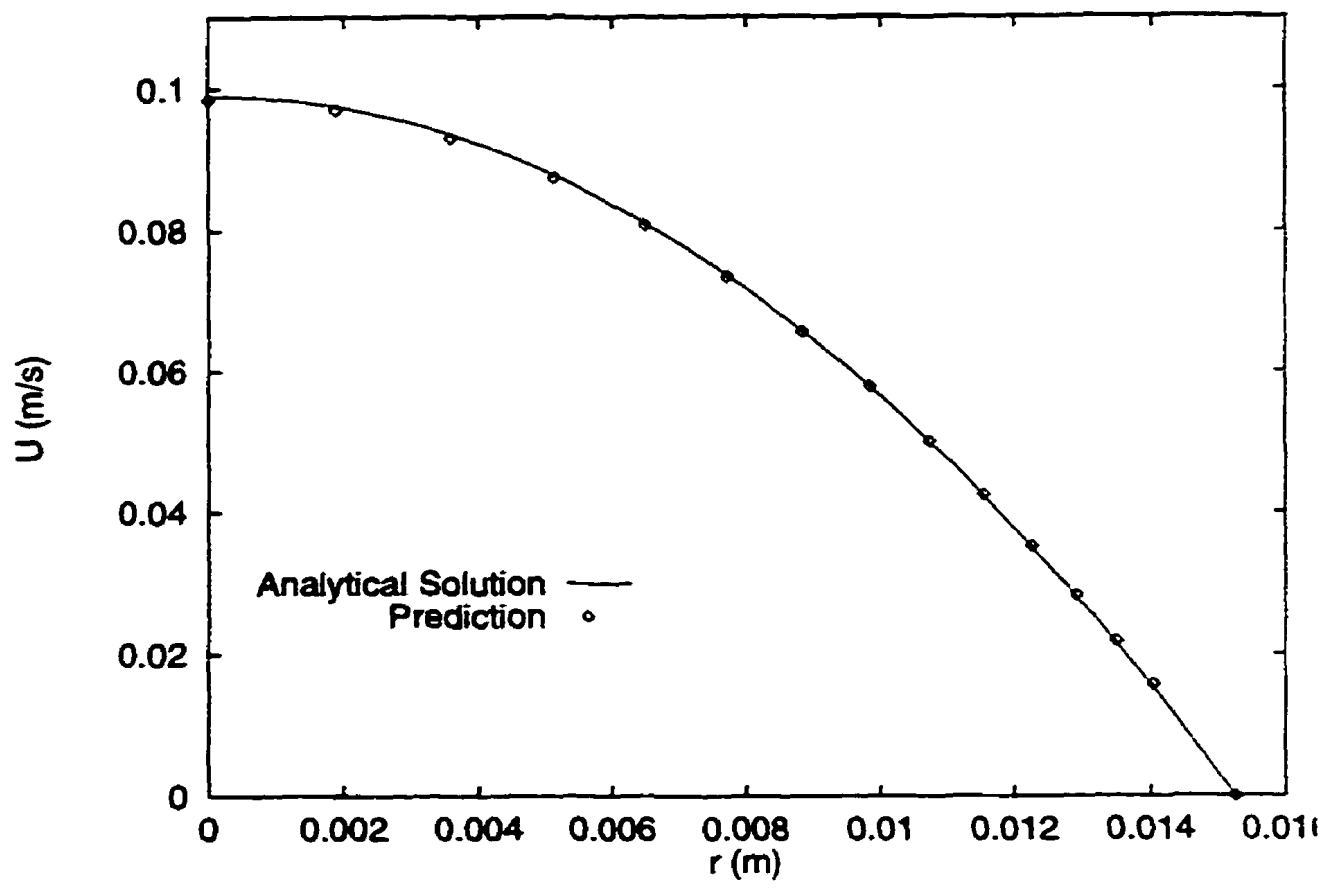

Figure 5.2: Axial velocity, analytical solution and code prediction using wedge-shaped grid, $R e=100$

\subsection{Turbulent Pipe Flow}

Turbulent pipe flow was the next stage of the validation procedure. This provided for the establishment of TASCflow's ability to predict 'clean' (single-phase) flows. Sincr the prediction will not improve with the addition of the particulate phase, these results provide a reference for the minimum error to be expected in more complex flows. Using the same grid as described above, the prediction was compared to the experimental results of Tsuji et al [44]. Both the velocity and turbulence intensity, $T_{i}$ profiles were obtained in the fully-developed region. The turbulence intensity is defined by;

$$
T_{i}=\frac{\sqrt{\frac{2}{3} k}}{U_{c}}
$$

where $U_{c}$ is the mean centerline velocity. The analytical solution for the axial relocity: $U$, for turbulent flow in a pipe is given by the power-law equation [33],

$$
U=U_{c}\left(1-\frac{r}{R}\right)^{\frac{1}{7}}
$$

This power-law profile cannot be used to obtain the slope at the wall $(y / r<0.04$. where $\mathrm{y}$ is equal to $R-r)$. In this region the profile yields $(\partial U / \partial y)_{\text {wall }}=\infty[13]$. The 
results obtained from the simulation are shown in Figures 5.3 and 5.4 .

Good agreement is obtained between the analytical solution and the experimental results and the velocity prediction. The turbulence intensity predictions over-predict the experimental values near the centerline and under-predict near the wall. The $L_{\text {.2.- }}$ norm error for the mean velocity is of $\mathrm{O}\left(10^{-3}\right)$ and for the turbulence intensity is of $O\left(10^{-2}\right)$. This magnitude of error is in keeping with the literature. Martinuzzi and Pollard [27] compared predictions obtained from the $k-\epsilon$ model with experimental data for developing pipe flow. It was found that the $k-\epsilon$ under-predicts the turbulent kinetic energy in the near-wall region. This under-prediction can be attributed to the anisotropic conditions in pipe flows. Since the $k-\epsilon$ model assumes isotropic conditions. the turbulence intensity of the predictions was calculated as $\sqrt{2 k / 3} / U_{c}$. The experimental turbulence intensity was reported as $u_{r m s} / U_{c}$. Since isotropic conditions do not exist in pipe flow, the turbulence intensity calculated from the prediction will under-predict the turbulence intensity reported in the experiment. This will be especially true near the wall where anisotropy is greatest in a pipe flow.

In order to establish grid-independence the grid was refined to 30 nodes in the ; direction, 3 nodes in the $j$ direction and 1700 nodes in the $k$ direction. This represents a $240 \%$ increase in the number of nodes. Figures 5.5 and 5.6 show that there was very little change in the flow solution when the fine grid was used. Therefore the flow solution obtained with the coarser grid ( $15 \times 3 \times 1000)$ is assumed to be grid-independent.

As discussed in Section 4.2.5, the turbulence intensity, $T_{i}$, and eddy length scale. $L$, are defined at the pipe inlet. The experimental data did not include information regarding inlet conditions, so these values had to be assumed. The TASCflow user documentation [3] suggests using values between 1 and $10 \%$ for the turbulence intensity and between 5 and $50 \%$ of the inlet characteristic length scale for the eddy length scale. The initial run shown in Figure 5.4 had as inlet conditions, $T_{i}=3 \%$ and $L_{\epsilon}=3 \%$ of the pipe diameter. The velocity and turbulence intensity profiles of Figures 5.7 and 5.8 show the effect of reducing the inlet turbulence intensity by $50 \%$, from $6 \%$ to $3 \%$. Figures 5.9 and 5.10 show the profiles for an increase of the inlet eddy length scale from $3 \%$ to $30 \%$ of the inlet characteristic length. As there is no significant change to the flow solution for either case it can be concluded that the flow solution is not sensitive to changes in inlet values of $T_{i}$ and $L_{\epsilon}$.

Recall from the Section 4.2.5 that the logarithmic-law of the wall is used to resolve the large gradients near the wall. The recommended practice is to locate the near-wall 


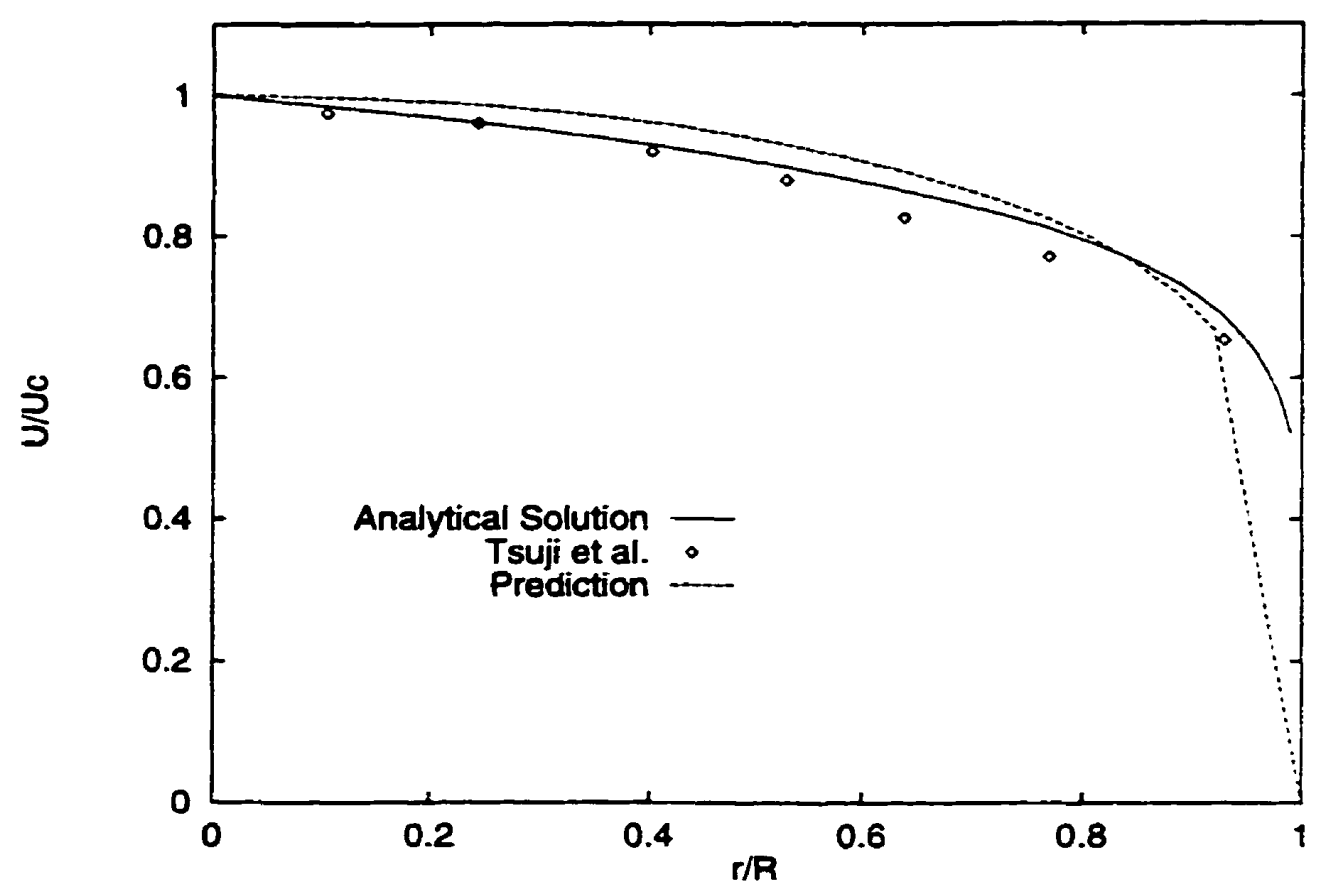

Figure 5.3: Mean velocity: Prediction of analytical solution and experimental data of Tsuji et al.[44], $R e \approx 23000$

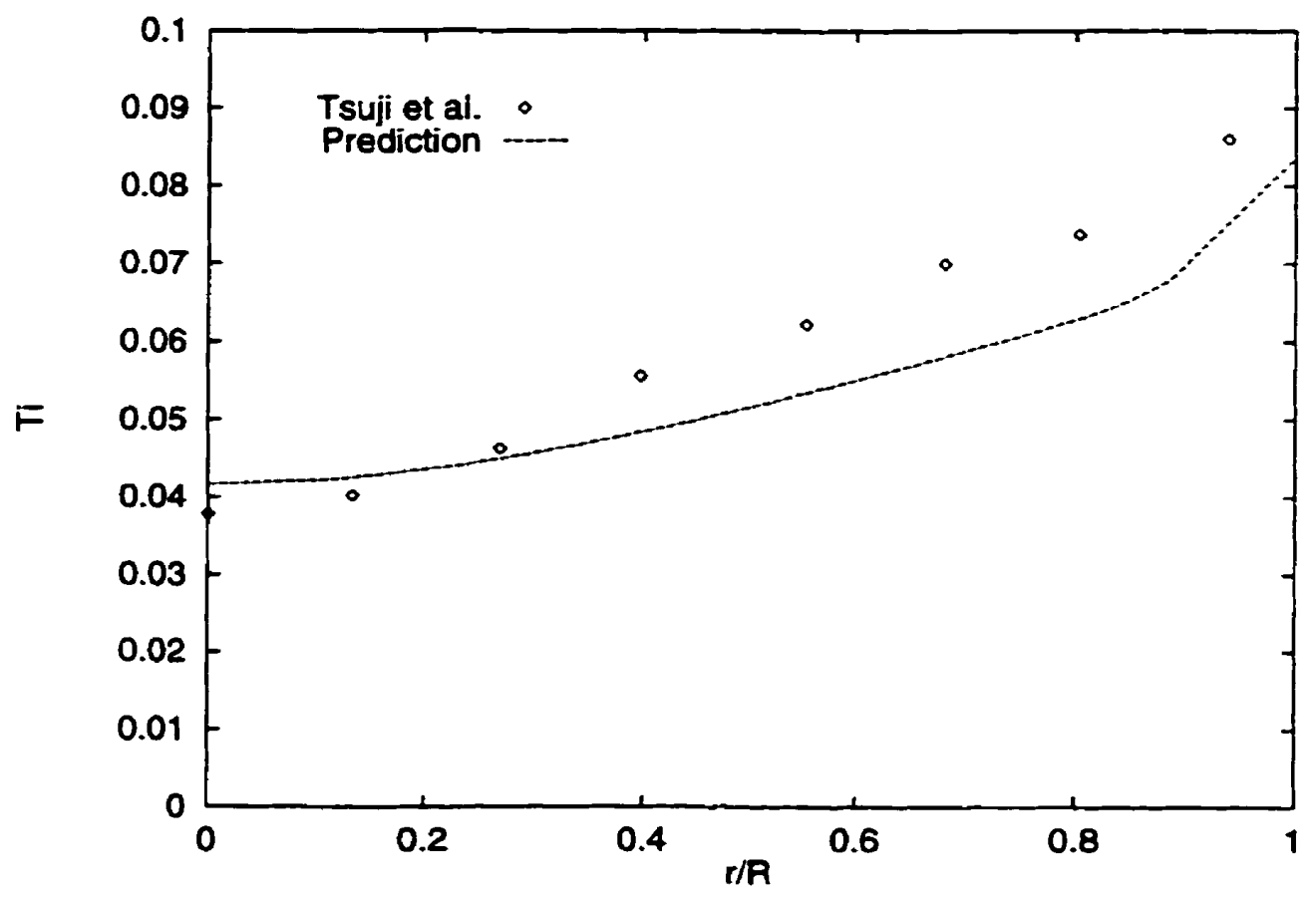

Figure 5.4: Turbulence intensity: Prediction of experimental data of Tsuji et al.[4]. $R e \approx 23000$ 


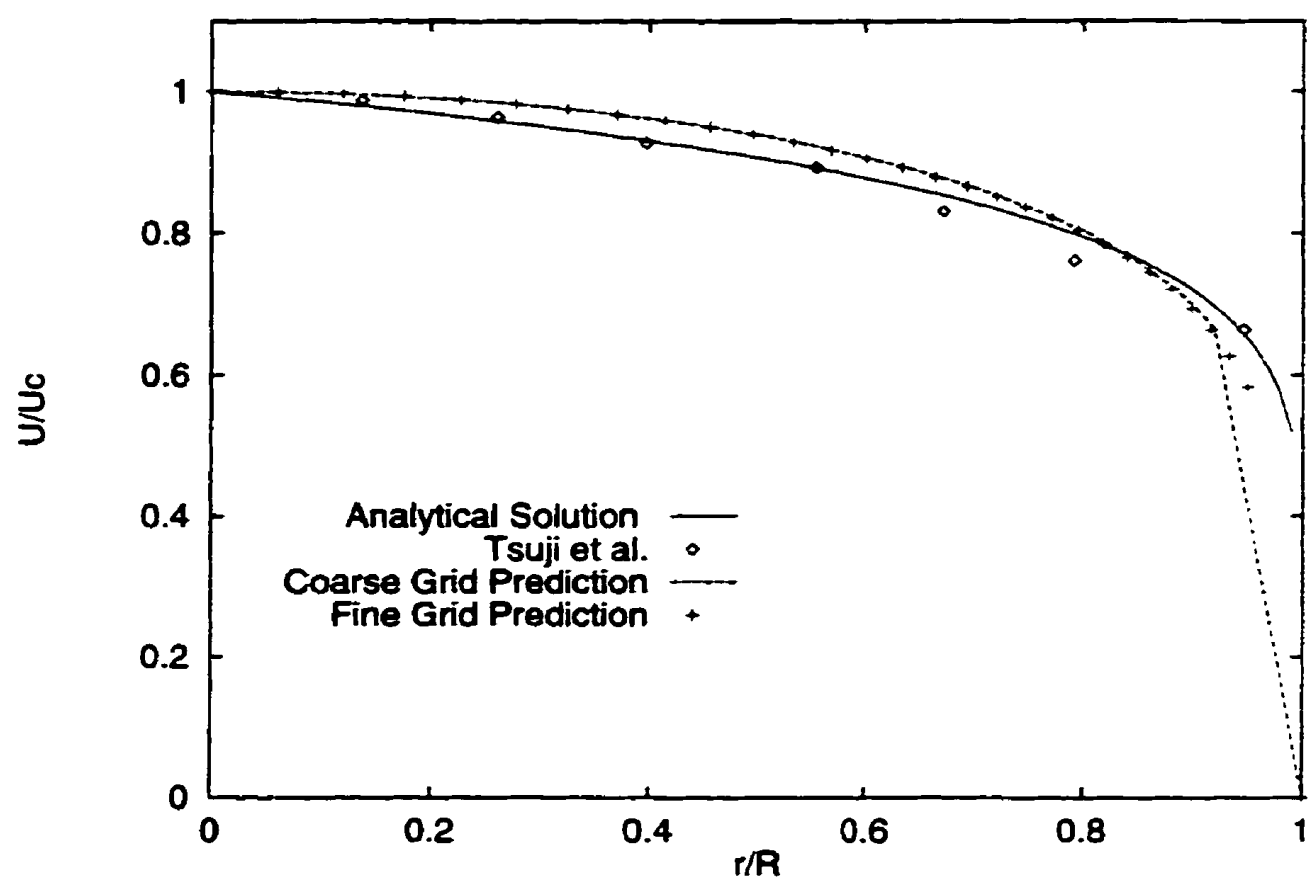

Figure 5.5: Mean velocity: Prediction using coarse $(15 \times 3 \times 1500)$ and fine $(30 \times 3 \times 1700)$ grids of analytical solution and experimental data of Tsuji et al.[44], $R e \approx 23000$

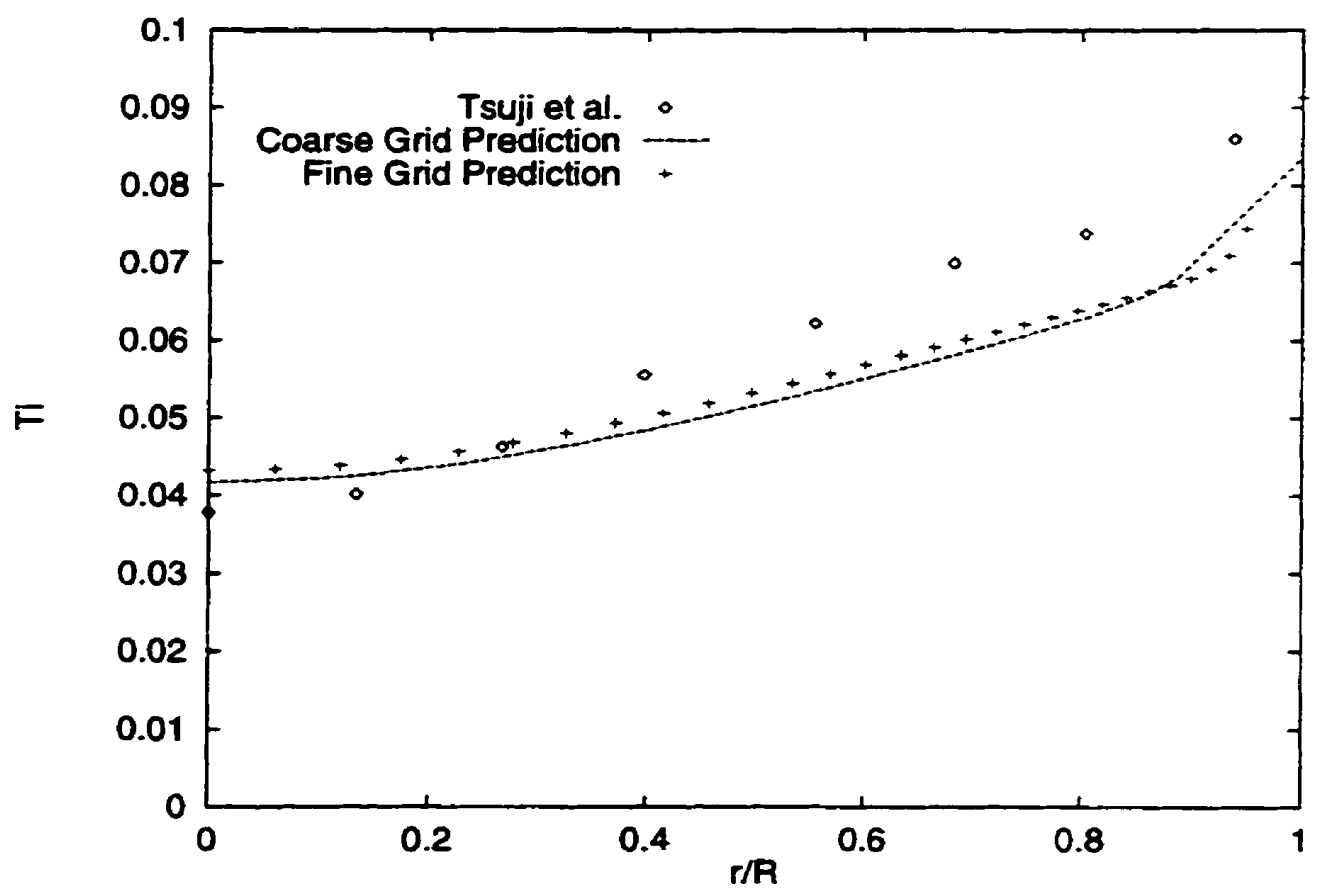

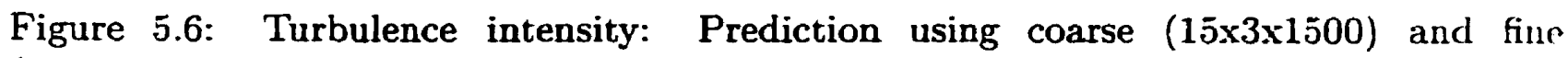

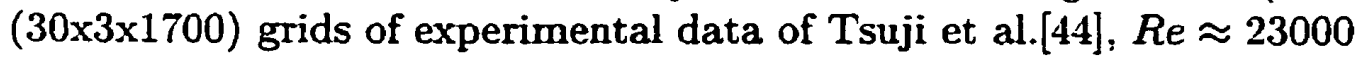




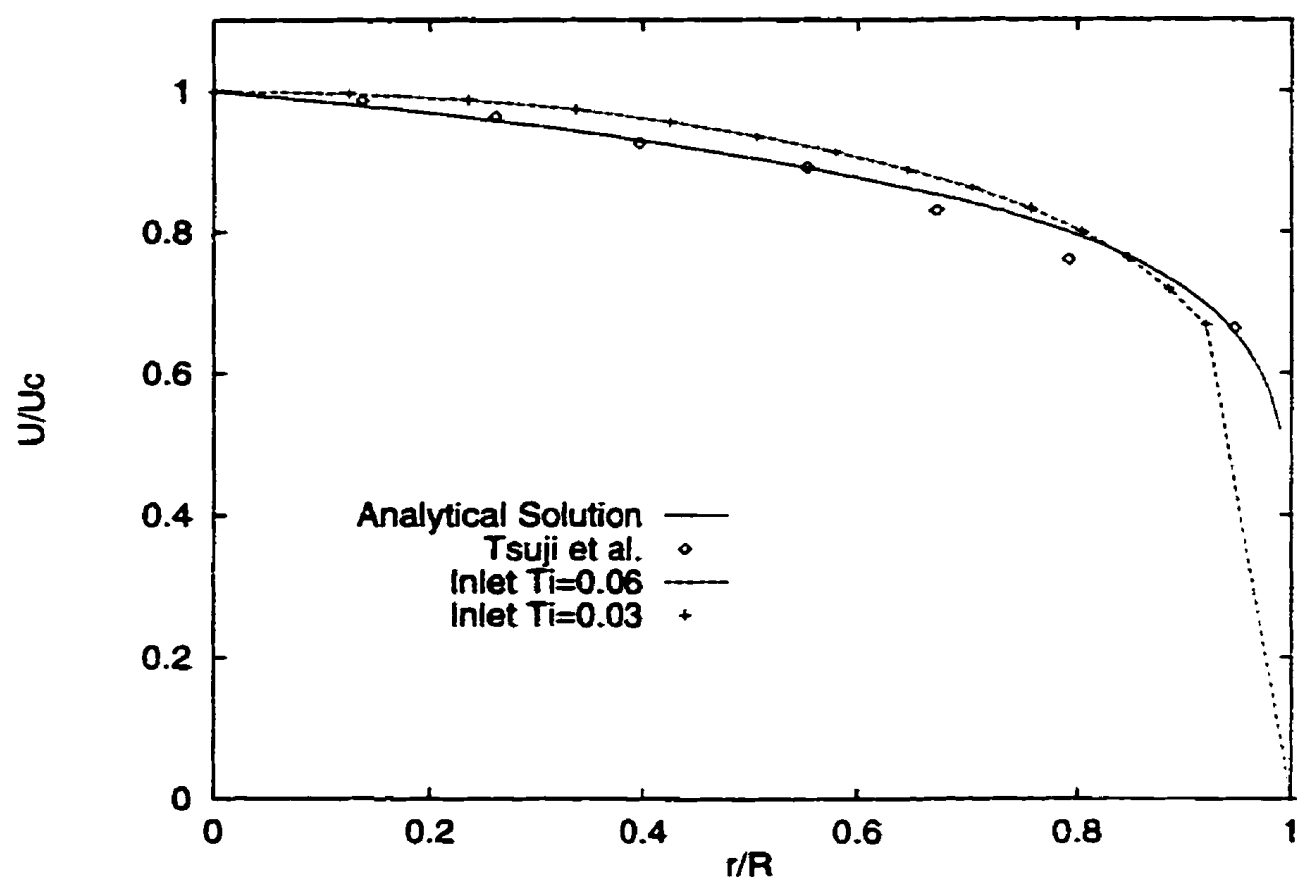

Figure 5.7: Mean velocity: Sensitivity of prediction to changes in inlet values of $T_{1}$. Prediction of analytical solution and experimental data of Tsuji et al.[44], $R e \approx 2: 3000$

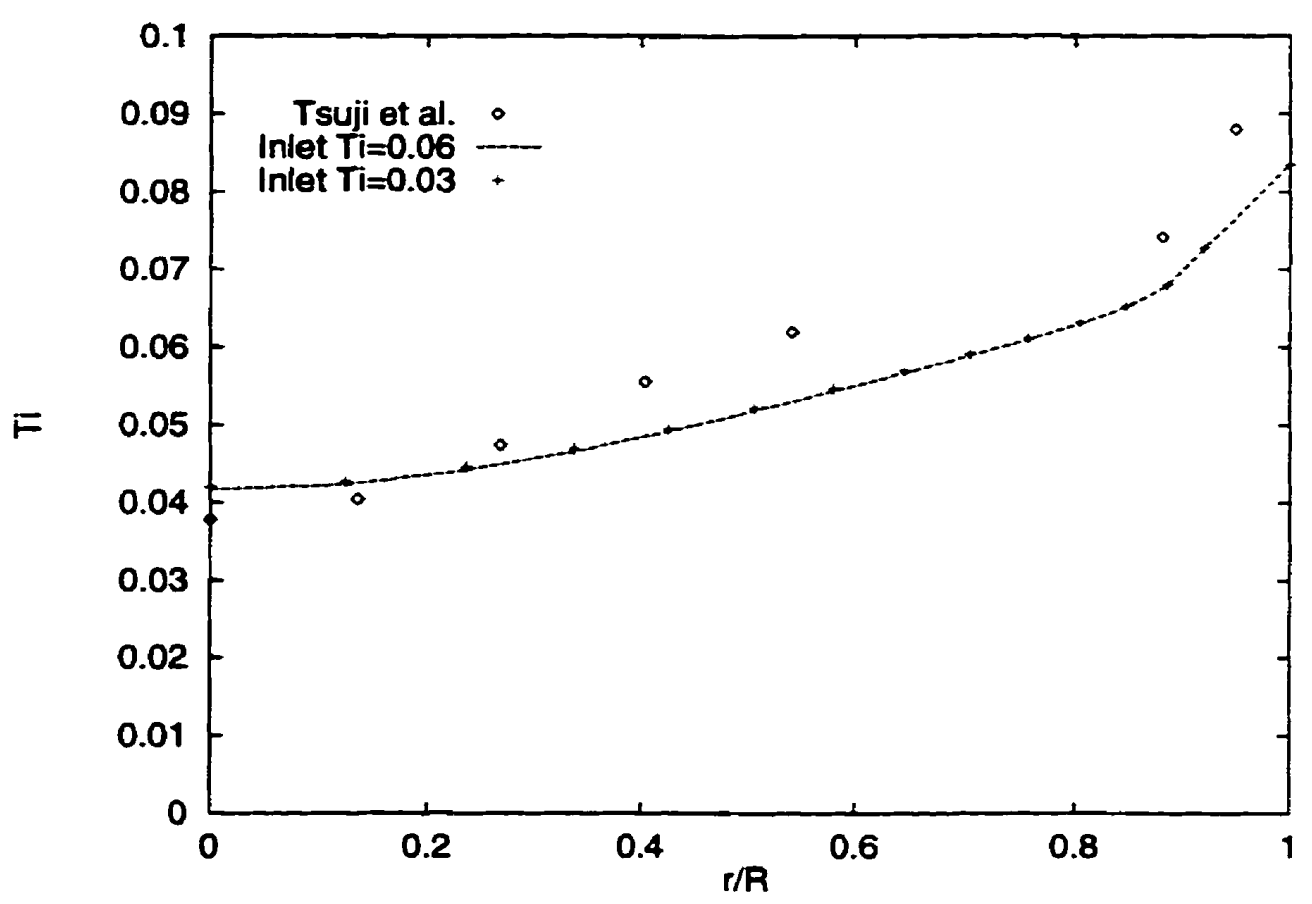

Figure 5.8: Turbulence intensity: Sensitivity of prediction to changes in inlet values of $T_{i}$. Prediction of experimental data of Tsuji et al.[44], $R e \approx 23000$ 


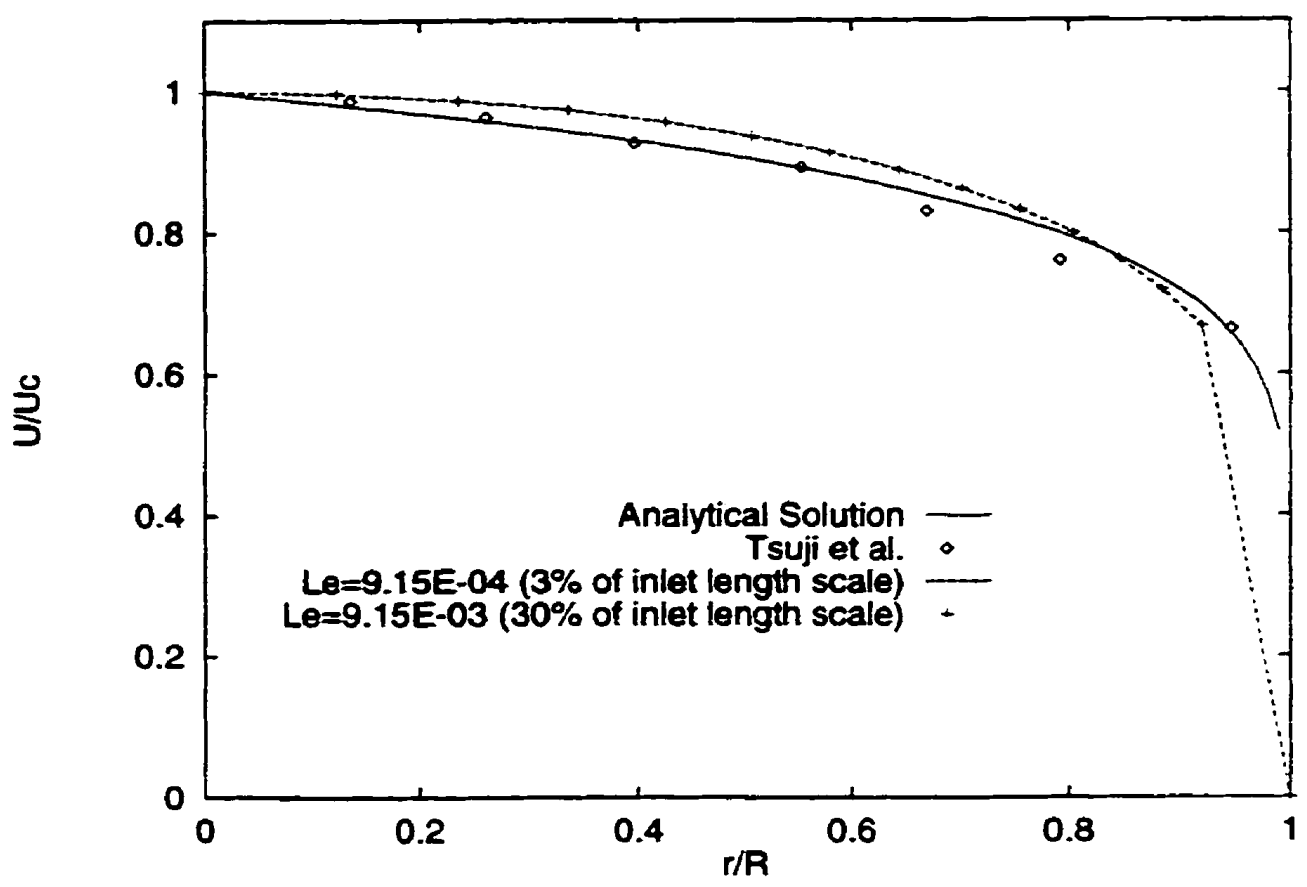

Figure 5.9: Mean velocity: Sensitivity of prediction to changes in inlet values of $L_{\text {. }}$. Prediction of analytical solution and experimental data of Tsuji et al.[44], $R e \approx 23000$

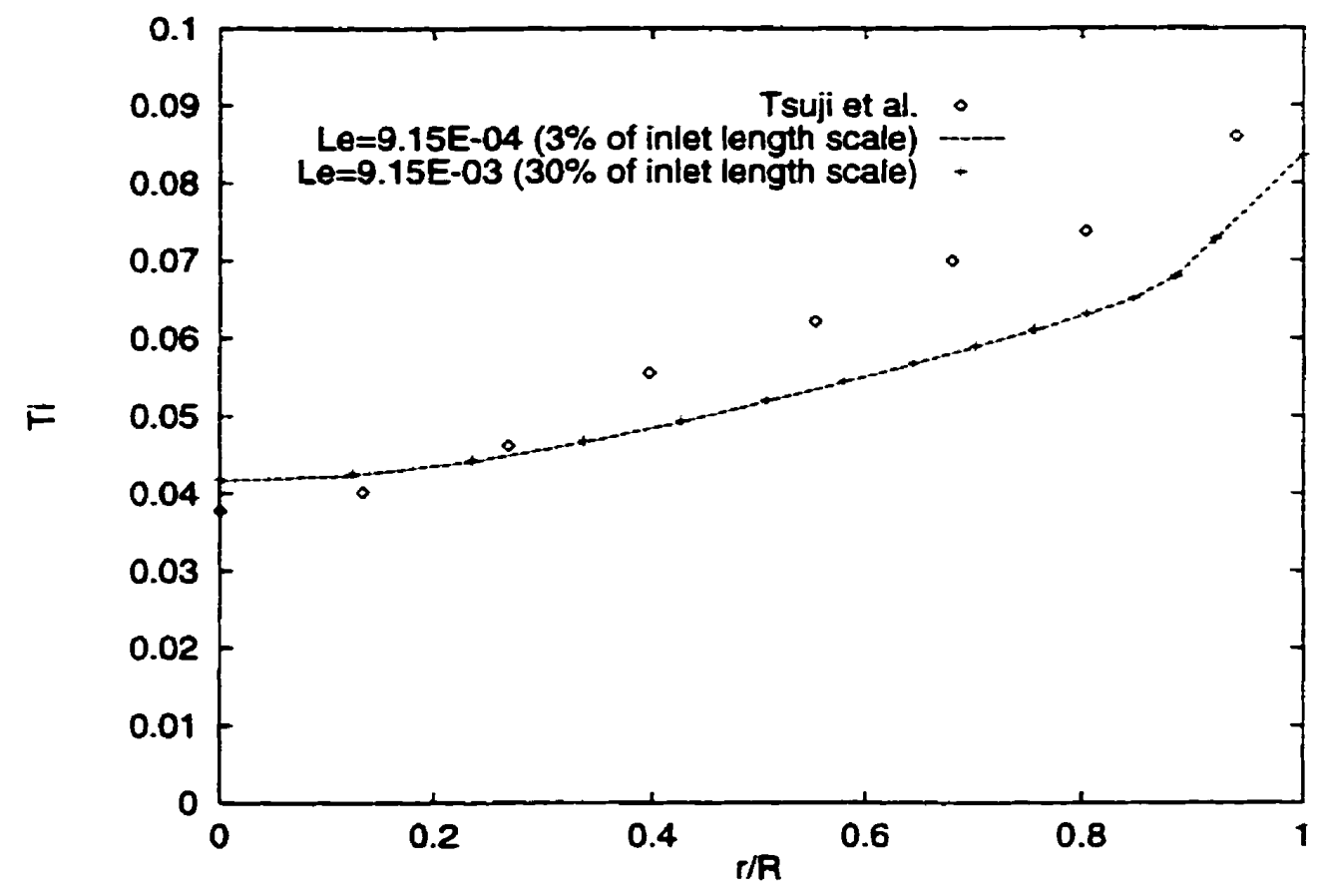

Figure 5.10: Turbulence intensity: Sensitivity of prediction to changes in inlet values of $L_{\epsilon}$. Prediction of experimental data of Tsuji et al.[44], $R e \approx 23000$ 


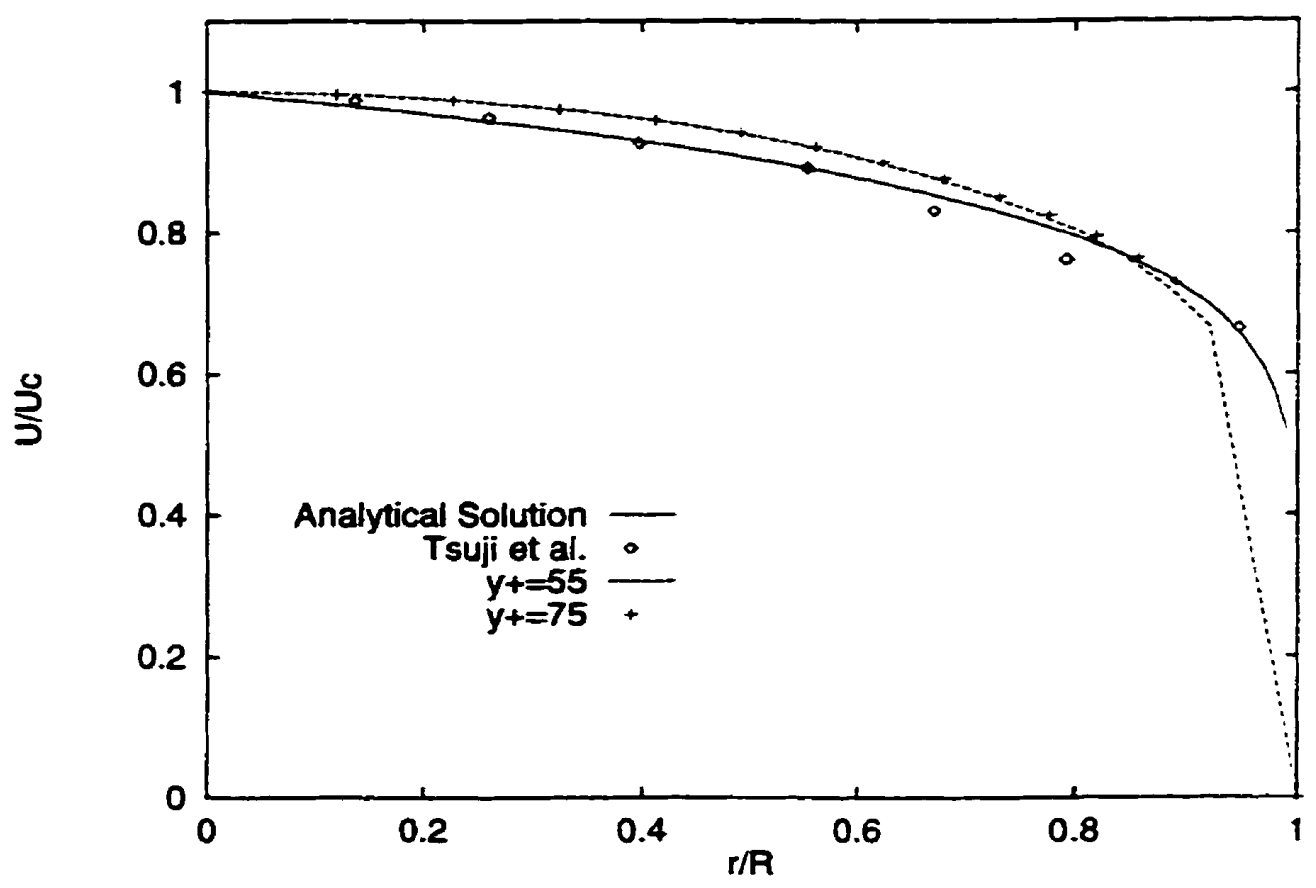

Figure 5.11: Mean velocity: Sensitivity of prediction to changes in $y^{\dagger}$. Prediction of analytical solution and experimental data of Tsuji et al.[44], $R e \approx 23000$

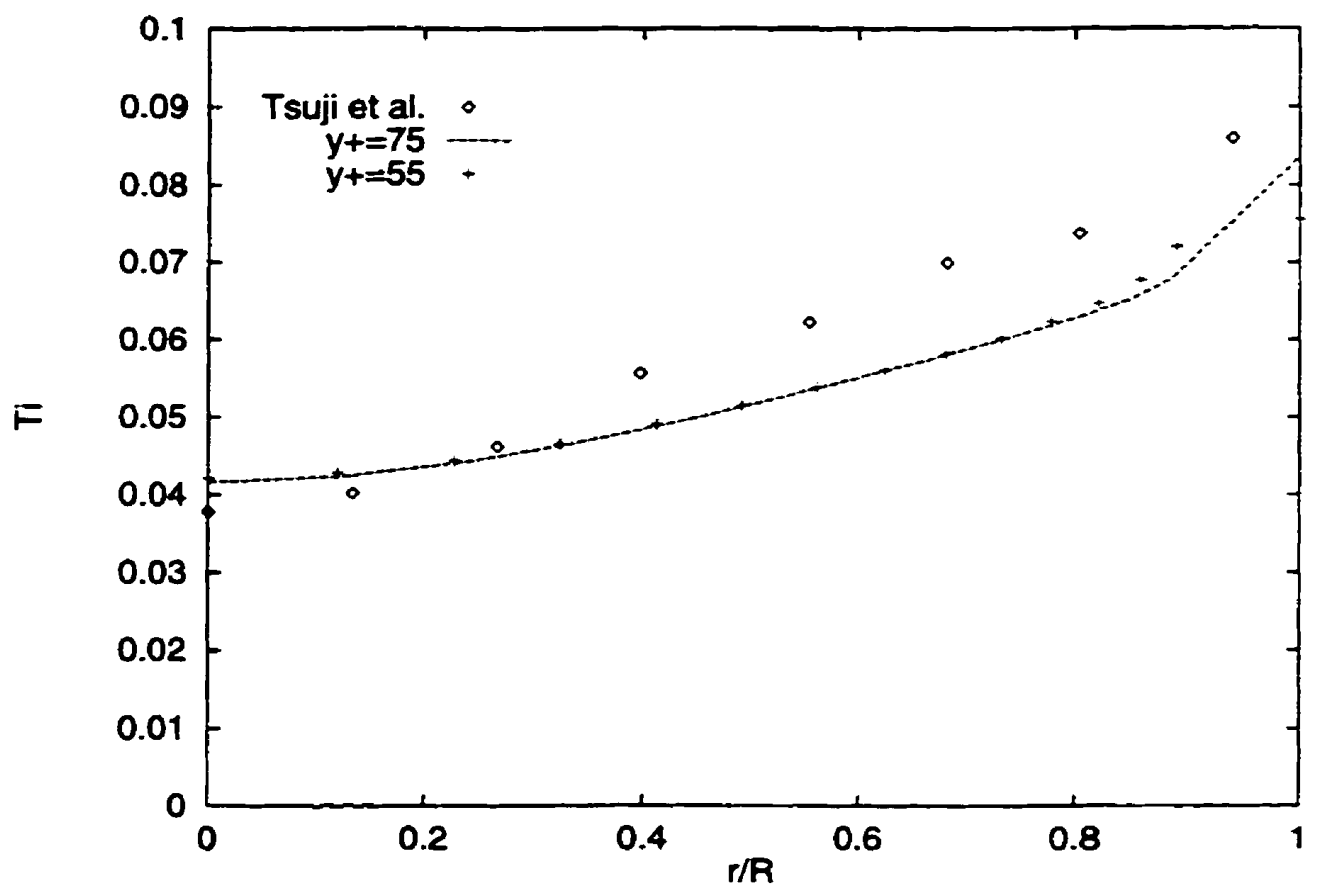

Figure 5.12: Turbulence intensity: Sensitivity of prediction to changes in $y^{\div}$. Prediction of experimental data of Tsuji et al.[44], $R e \approx 23000$ 


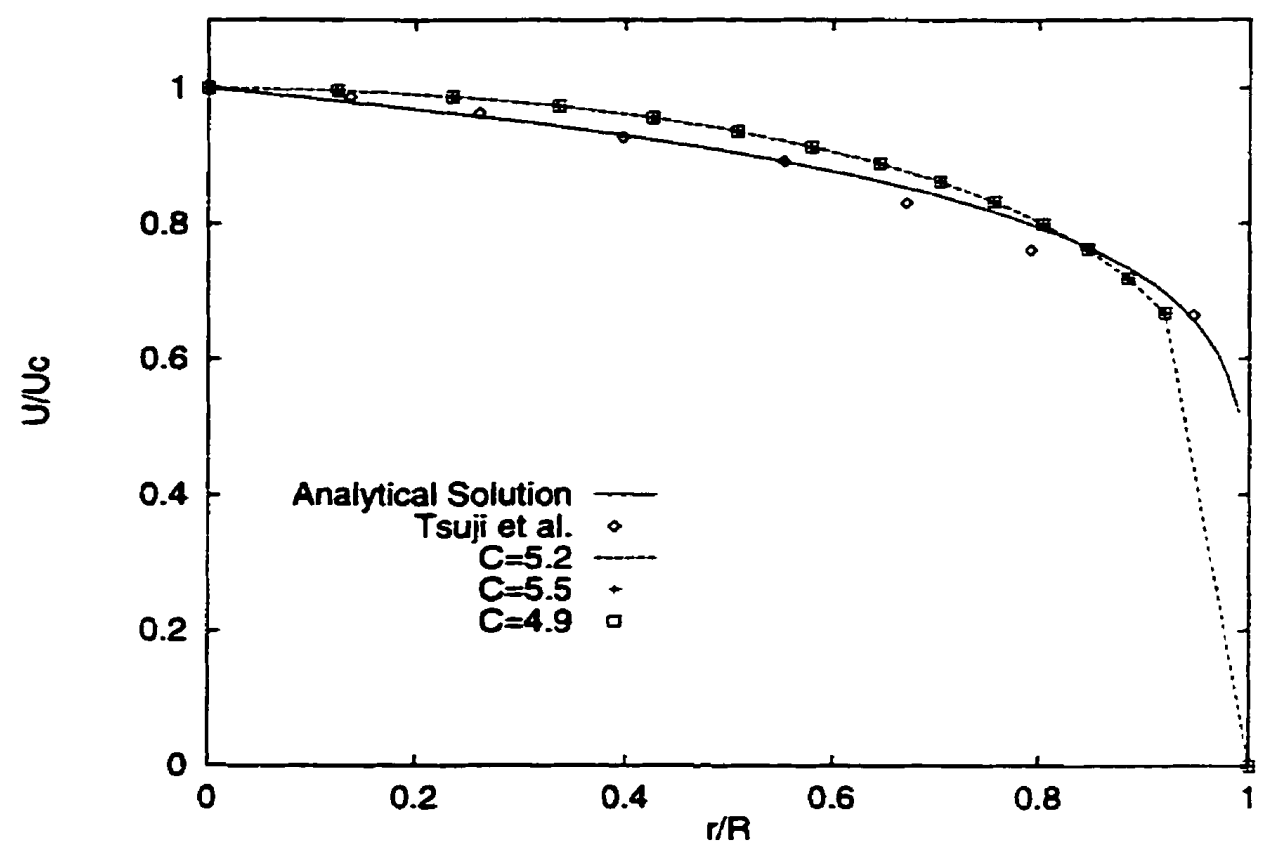

Figure 5.13: Mean velocity: Sensitivity of prediction to changes log-law of the wall constant, $C$. Prediction of analytical solution and experimental data of Tsuji et al. $[4+]$. $R e \approx 23000$

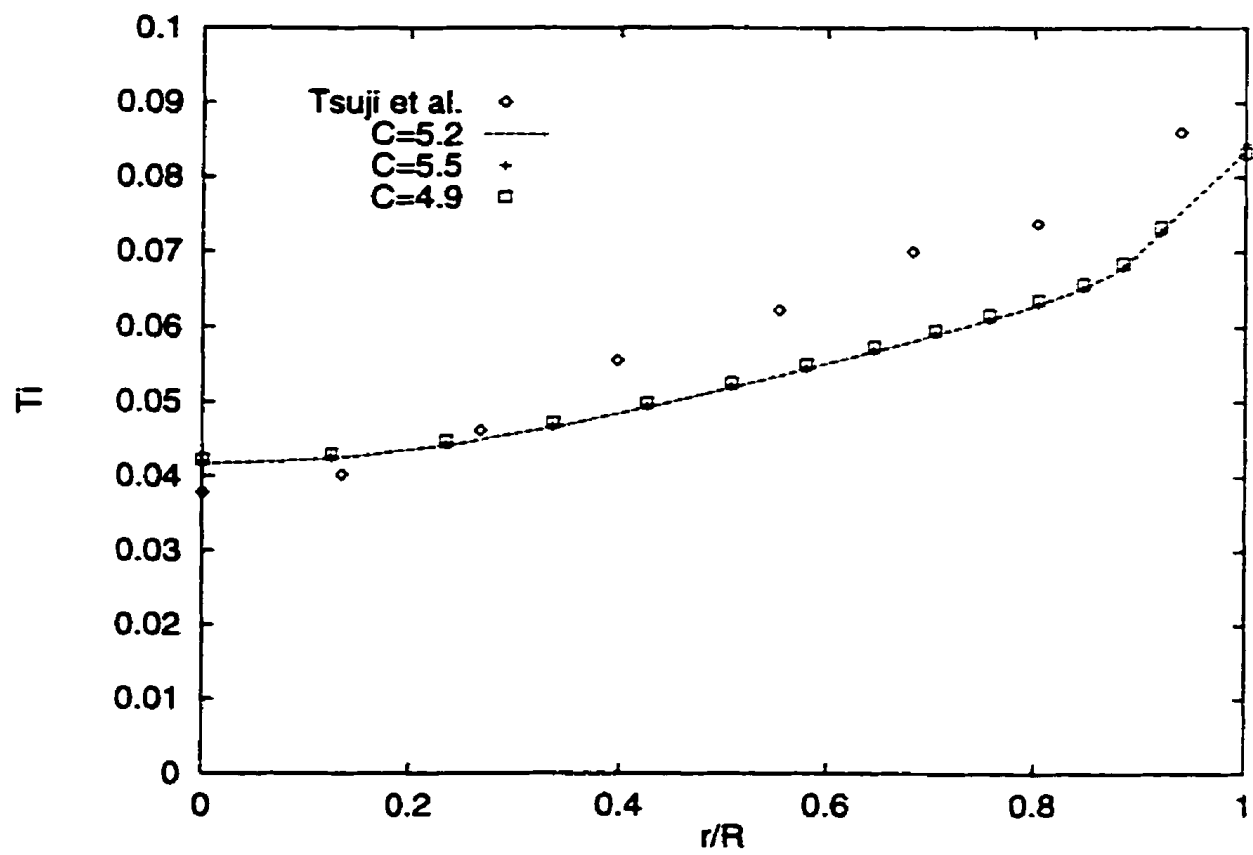

Figure 5.14: Turbulence intensity: Sensitivity of prediction to changes in log-law of the wall constant, $C$. Prediction of experimental data of Tsuji et al.[44], $R e \approx 23000$ 
Table 5.1: Summary of Sensitivity Analysis

\begin{tabular}{|l|c|c|}
\hline & \multicolumn{2}{|c|}{ Values } \\
\hline Number of Nodes & 45000 & 153000 \\
\hline$T_{i}$ & $3 \%$ & $6 \%$ \\
\hline$L_{\epsilon}$ & $3 \%$ & $30 \%$ \\
\hline$y^{+}$ & 55 & 75 \\
\hline$C$ & 5.2 & $4.9,5.5$ \\
\hline
\end{tabular}

node such that $y^{+}$is in the range of 30 to 500 [2], where

$$
y^{+}=\frac{C_{\mu}^{1 / 4} \sqrt{k} \Delta n}{\nu}
$$

and $\Delta n$ is the distance from the wall to the near-wall node.

Figures 5.11 and 5.12 show the effect of varying $y^{+}$on the solution. In the first run. $y^{+}$had a value of 55 while in the second run the near-wall node was moved farther awas from the wall to the corresponding $y^{+}$value of 75 . There is no significant change to the flow solution as a result of varying the near-wall node placement.

The final parameter that was examined was the effect of the constant that appears in the log-law of the wall. Recall that the near-wall tangential velocity is related to the wall shear stress through the standard wall function,

$$
u^{+}=\frac{1}{0.41} \ln \left(y^{+}\right)+C
$$

$C$ has a default value in TASCflow of 5.2 , however literature suggests that values ranging from 4.9 [33] to 5.5 [36] are acceptable. In order to verify the sensitivity of the solution to the value of $C$, simulations were performed with values of $C$ set to $4.9,5.2$ and 5.5 . Figures 5.13 and 5.14 show that the value of $C$ chosen in the range form 4.9 to 5.5 does not significantly affect the prediction.

Table 5.1 provides a summary of the sensitivity analysis performed for the turbulent pipe flow simulations. The second column corresponds to the values that were held constant for all of the analyses.

\subsection{Particle Dispersion}

All turbulence modulation models require knowledge of the particle concentration in the flow. Before proceeding on to model turbulence modulation, it had to be rerified that 


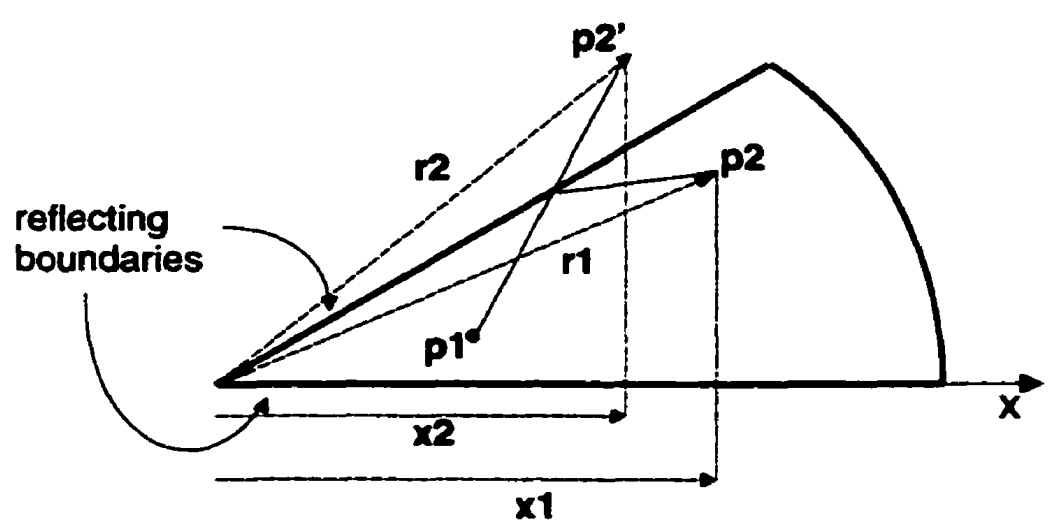

Figure 5.15: Particle path in a wedge-shape geometry

the flow could properly predict the location of the particles in a pipe flow; or rather. properly predict the particle dispersion.

The 1988 experiment of researchers Vames and Hanratty [46] was selected to validate TASCflow's predictions. This experiment involved a downward turbulent air flow into which water droplets were injected from a point source located at the center of the pipe. The particle dispersion was reported in the literature as $\overline{x^{2}}$, the "mean-squared displacement normal to the direction of the mean flow."

The wedge-shaped grid described in Section 5.1 was used in this validation. This presented an interesting problem: In a wedge-shaped geometry the distribution of particle position with respect to $x$ and $y$ is not known. Instead, the distribution of the radial displacement of the particle is known. Referring to Figure 5.15, consider the case of a particle starting at $p I$ and reflecting off a radial boundary. After reflecting, the particle is at $p 2$ with a radial location of $r_{1}$ and an $x$-location of $x_{1}$. Had the particle not been reflected it would be at $p 2^{\prime}$ with a radial location of $r_{2}$ and an $x$-location of $x_{2}$. Since the boundary is perfectly reflecting $r_{1}=r_{2}$, however it is clear that $x_{1}$ does not equal $x_{2}$. Therefore, in order to correctly interpret the computational data the relationship between radial particle distribution and Cartesian particle distribution must be established.

Consider the circular cross-section shown in Figure 5.16. Let $f_{r}(r)$ be the probability density function (PDF) for the particle radial position. The probability of a particle having a radial location in the small interval centered at $R^{*}$ is:

$$
\text { Probability that } r \in\left(R^{*}-\frac{\Delta r}{2}, R^{*}+\frac{\Delta r}{2}\right)=f_{r}\left(R^{*}\right) \triangle r
$$

If ' $N$ ' particles are released into the flow, the number of particles expected in the ring 


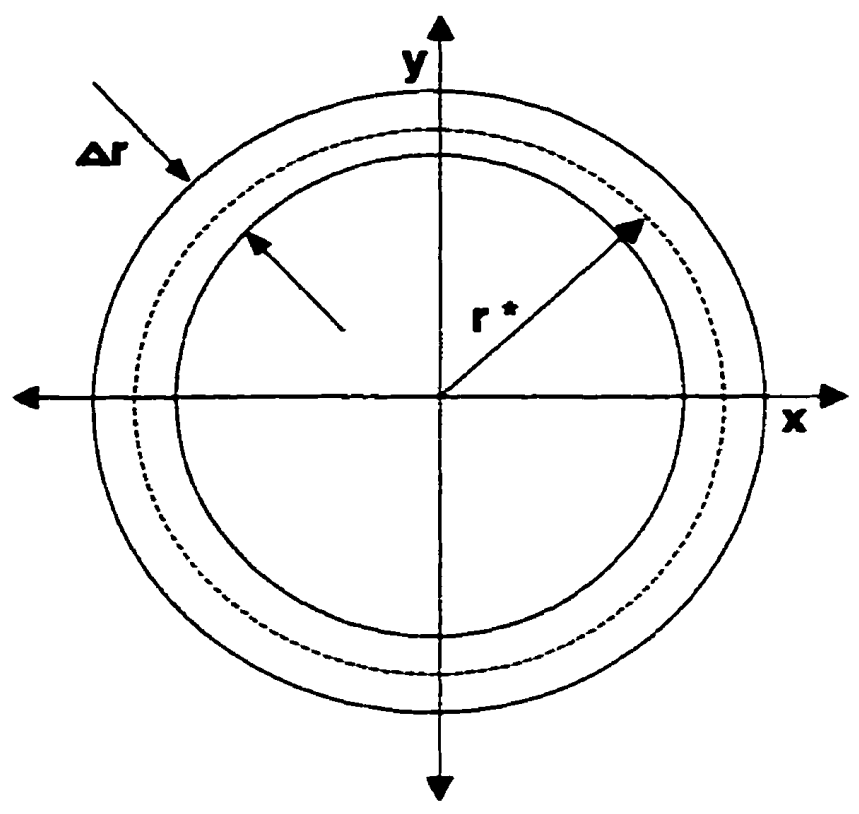

Figure 5.16: Circular Cross-Section

will be

$$
N f_{r}\left(R^{*}\right) \triangle r
$$

and the concentration of the particles in the ring, $C\left(R^{*}\right)$, is given by:

$$
\begin{aligned}
C\left(R^{*}\right) & =\frac{\text { number of particles }}{\text { volume }} \\
& =\frac{N f_{r}\left(R^{*}\right) \Delta r}{2 \pi R^{*} \Delta r} \\
& =\frac{N f_{r}\left(R^{*}\right)}{2 \pi R^{*}}
\end{aligned}
$$

If $C\left(R^{*}\right)$ is divided by $N$ the expression will integrate to unity (a property of a PDF). Now the expression for the normalized concentration of particles in the ring is actuallythe same as a joint $x-y$ PDF for the particles in Cartesian space. Therefore.

$$
\begin{aligned}
f_{x y}(x, y) & =C\left(R^{*}\right) \\
& =C\left(\sqrt{x^{2}+y^{2}}\right) \\
& =\frac{f_{r}\left(\sqrt{x^{2}+y^{2}}\right)}{2 \pi \sqrt{x^{2}+y^{2}}}
\end{aligned}
$$


So a PDF for $x$ is given by,

$$
\begin{aligned}
f_{x}(x) & =\int_{-\infty}^{\infty} f_{x y}(x, y) d y \\
& =\int_{-\infty}^{\infty} \frac{f_{r}\left(\sqrt{x^{2}+y^{2}}\right)}{2 \pi \sqrt{x^{2}+y^{2}}} d y
\end{aligned}
$$

This is the desired relationship between PDFs in Cartesian and Cylindrical coordinate systems. Lightstone [24] verified this result for particles in homogeneous, isotropic turbulence. Lightstone found that the PDF for radial particle position is described bythe Weibull distribution,

$$
f_{r}(r)=\frac{r}{\sigma^{2}} e^{\frac{-r^{2}}{2 \sigma^{2}}}
$$

where $\sigma^{2}=2 \varepsilon t$. It is also known that for particles in homogeneous, isotropic turbulence. $f_{x}(x)$ has a Gaussian distribution with a variance of $\sigma^{2}=2 \varepsilon t$. So if Equation ( 5.10 ) is correct a Gaussian distribution must be obtained when the PDF for radial particle position is used. Substituting Equation (5.11) into Equation (5.10) gives,

$$
\begin{aligned}
f_{x}(x) & =\int_{-\infty}^{\infty} \frac{f_{r}\left(\sqrt{x^{2}+y^{2}}\right)}{2 \pi \sqrt{x^{2}+y^{2}}} d y \\
& =\int_{-\infty}^{\infty} \frac{\sqrt{x^{2}+y^{2}}}{\sigma^{2} 2 \pi \sqrt{x^{2}+y^{2}}} e^{-\frac{x^{2}+y^{2}}{2 \sigma^{2}}} d y \\
& =\int_{-\infty}^{\infty} \frac{1}{2 \pi \sigma^{2}} e^{-\frac{x^{2}}{2 \sigma^{2}}} e^{-\frac{y^{2}}{2 \sigma^{2}}} d y \\
& =\frac{1}{\sqrt{2 \pi \sigma^{2}}} e^{-\frac{x^{2}}{2 \sigma^{2}}} \underbrace{\int_{-\infty} \frac{1}{\sqrt{2 \pi \sigma^{2}}} e^{-\frac{y^{2}}{2 \sigma^{2}}} d y}_{-\infty} \\
& =\frac{1}{\sqrt{2 \pi \sigma^{2}}} e^{-\frac{x^{2}}{2 \sigma^{2}}}
\end{aligned}
$$

which is a Gaussian distribution in $x$ with variance equal to $\sigma^{2}$.

Now $\overline{x_{p}^{2}}$ can be determined from the relationship between Cartesian and Cylindrical coordinate systems, Eq.(5.10):

$$
\begin{aligned}
\overline{x_{p}^{2}} & =\int_{-\infty}^{\infty} x^{2} f_{x}(x) d x \\
& =\int_{-\infty}^{\infty} x^{2} \int_{-\infty}^{\infty} \frac{f_{r}\left(\sqrt{x^{2}+y^{2}}\right)}{2 \pi \sqrt{x^{2}+y^{2}}} \underbrace{d y d x}_{d A}
\end{aligned}
$$


Using the following change of variables,

$$
\begin{aligned}
x & =r \cos \theta \\
y & =r \sin \theta \\
x^{2}+y^{2} & =r^{2} \\
d A & =r d r d \theta
\end{aligned}
$$

and substituting into Equation (5.13) yields,

$$
\begin{aligned}
\overline{x^{2}} & =\int_{-\infty}^{\infty} \int_{0}^{2 \pi} r^{2} \cos ^{2} \theta \frac{f_{r}(r)}{2 \pi r} r d r d \theta \\
& =\frac{1}{2} \int_{-\infty}^{\infty} r^{2} f_{r}(r) d r \\
& =\frac{\overline{r^{2}}}{2}
\end{aligned}
$$

This is the expression which relates radial particle displacement to Cartesian particle displacement. Figure 5.17 shows the result when this expression is applied to the data from the simulation and compared with Vames and Hanratty's experimental results. The result obtained from TASCflow under-predicts the results of Vames and Hanrattr: however the slope is in good agreement.

\subsubsection{Relevance to Taylor's Analysis}

Taylor [42] mathematically described the dispersion of a small particle from a point source in stationary, homogeneous, isotropic turbulence. Using this analysis a turbulent diffusion coefficient, $\varepsilon_{p}$, can be determined. This coefficient describes the dispersion of the particles in the turbulent flow.

Taylor's analysis shows that for large times the variance of the particle displacement is linear with time such that

$$
\frac{1}{2} \frac{d \overline{x^{2}}}{d t}=\overline{u_{p}^{\prime 2}} \tau_{L I}=\varepsilon_{p}
$$

where $\overline{x^{2}}$ is the variance of the particle displacement normal to the direction of the mean flow, $v_{p}^{\prime}$ represents the particle velocity fluctuations, and $\tau_{L I}$ is the Lagrangian integral timescale which characterizes the duration of the movement of a fluid particle in a given direction.

Substituting the relationship between radial and Cartesian particle displacement. 


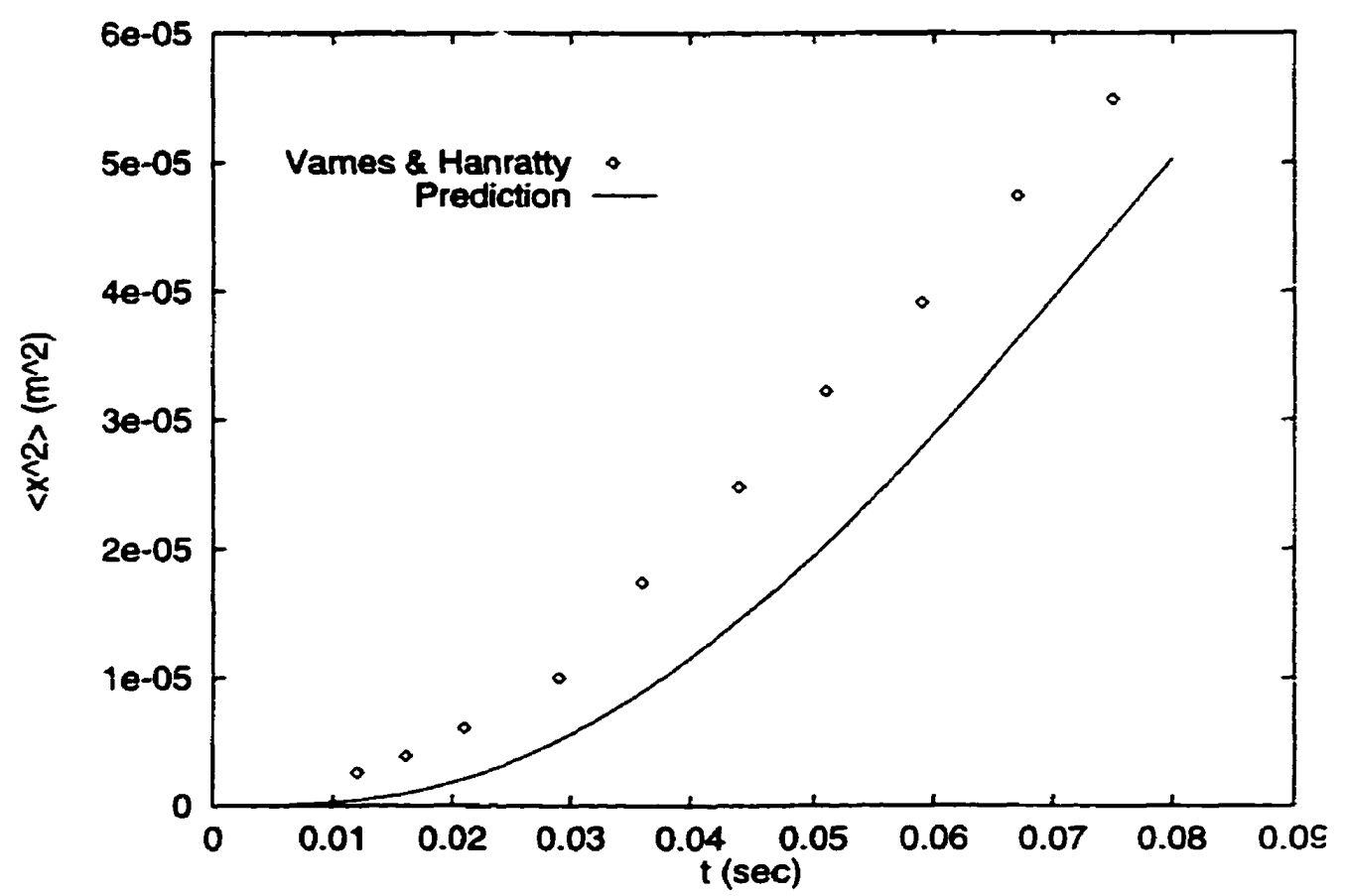

Figure 5.17: Particle Dispersion: Prediction of Vames and Hanratty's [46] experimental results using $\overline{x^{2}}=\overline{r^{2}} / 2$,

Equation (5.14), into Equation 5.15 gives:

$$
\frac{1}{4} \frac{d \overline{r^{2}}}{d t}=\varepsilon_{p}
$$

Referring again to Figure 5.17, even though the particle dispersion prediction underpredicts the experimental results of Vames and Hanratty the correct slope is obtained. Thus, Taylor's turbulent diffusion coefficient can be correctly obtained from the simulattion.

\subsection{Turbulent Source Term Implementation}

The purpose of this validation was to ensure that the source terms to the $k$ and $\epsilon$ equations were being implemented correctly. As described in Chapter 4 the source terms to the $k$ and $\epsilon$ equations are implemented through a user modifiable source code. Because the source terms are added to their respective equations through calls to subroutines, the user modifiable source code behaves as a 'black box'. It was therefore necessary to develop a verification procedure to ensure the source terms to the $k$ and $\epsilon$ equations were correctly. implemented. 
A grid-generated turbulent flow field was selected as the validating flow. This flow was chosen because an analytical solution to the $k$ and $\epsilon$ equations with source ternis could be easily derived for this problem. In a grid-generated turbulent flow field the flow is stationary, there is no production of turbulent kinetic energy and there are no gradients in the plane parallel to the inlet plane. This reduces the $k$ and $\epsilon$ equation to the following expressions:

$$
\begin{aligned}
U \frac{\partial k}{\partial x} & =-\epsilon \\
U \frac{\partial \epsilon}{\partial x} & =-C_{\epsilon 2} \frac{\epsilon^{2}}{k}
\end{aligned}
$$

where $U$ is the mean flow velocity in the axial direction, $x$. The analytical solution is given by:

$$
\begin{aligned}
k & =k_{o}\left(1+\frac{x}{U a}\right)^{-n} \\
\epsilon & =\epsilon_{o}\left(1+\frac{x}{U a}\right)^{-(n+1)}
\end{aligned}
$$

where $k_{o}$ and $\epsilon_{o}$ represent the initial values of $k$ and $\epsilon$ respectively and $a$ and $n$ are giren by,

$$
\begin{aligned}
a & =\frac{n k_{o}}{\epsilon_{o}} \\
n & =\frac{1}{C_{\epsilon 2}-1}
\end{aligned}
$$

Suppose a source term of magnitude $-b \epsilon$ is added to the expression for $k$, where $b$ is a constant. The equations for $k$ and $\epsilon$ become,

$$
\begin{aligned}
& U \frac{\partial k}{\partial x}=-\epsilon-b \epsilon \\
& U \frac{\partial \epsilon}{\partial x}=-C_{\epsilon 2} \frac{\epsilon^{2}}{k}
\end{aligned}
$$

Substituting $\epsilon^{*}=(1+b) \epsilon$ and $\partial \epsilon^{*}=(1+b) \partial \epsilon$ into the above equations gives.

$$
U \frac{\partial k}{\partial x}=-\epsilon^{*}
$$




$$
U \frac{\partial \epsilon^{*}}{\partial x}=-C^{*} \frac{\epsilon^{* 2}}{k}
$$

where $C^{*}=C_{\epsilon 2} /(1+b)$.

The solution for $k$ and $\epsilon$ is,

$$
\begin{aligned}
& k=k_{o}\left(1+\frac{x}{U a^{*}}\right)^{-n^{*}} \\
& \epsilon=\epsilon_{o}\left(1+\frac{x}{U a^{*}}\right)^{-\left(n^{*}+1\right)}
\end{aligned}
$$

where $a^{*}$ and $n^{*}$ are now given by,

$$
\begin{aligned}
a^{*} & =\frac{n^{*} k_{o}}{\epsilon_{o}^{*}} \\
n^{*} & =\frac{1}{C^{*}-1}
\end{aligned}
$$

A source in the form of $-b \epsilon$ was added the $k$ equation through the user modifiable source code, with $b$ taking on values of -0.5 and +0.5 . The predictions from the code are in excellent agreement with the analytical solution as shown in Figures 5.18 and 5.19 . It is therefore concluded that the user modifiable source code functions as is should.

\subsection{Momentum Source Term Implementation}

The final validation performed on the code was to ensure that the particle source terms to the momentum equation were correctly implemented in TASCflow. The portion of the source code where the momentum source terms are implemented was not available for verification, however the average momentum source term over the entire computational space is part of TASCflow's standard output and can be checked using the analytical solution of the momentum source term.

In order to match the output from TASCflow with the analytical solution a validation run was designed such that all of the variables appearing in the expression for the momentum source term were known. The validation run involved the injection of one particle into a laminar flow field. Because the flow was laminar the particle traveled along a straight path (constant $i, j$ ) through the domain. To ensure that the particle had a significant effect on the the gas-phase velocity, the particle was given a high mass flux $\left(\dot{m}_{p}=10 \mathrm{~kg} / \mathrm{s}\right)$ and an initial velocity that was $1 \%$ of the gas-phase. 


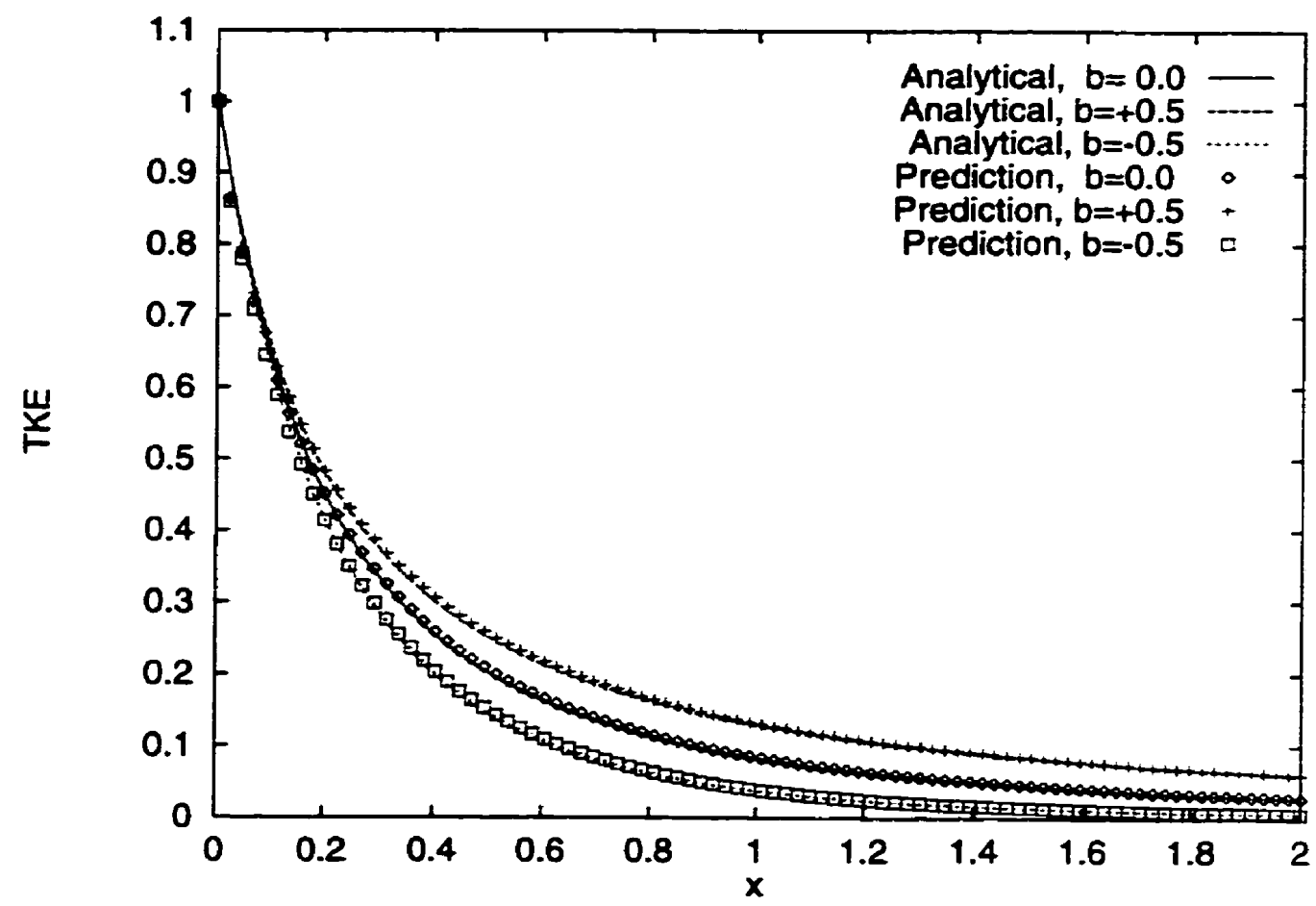

Figure 5.18: Analytical solution and code predictions for $\epsilon$

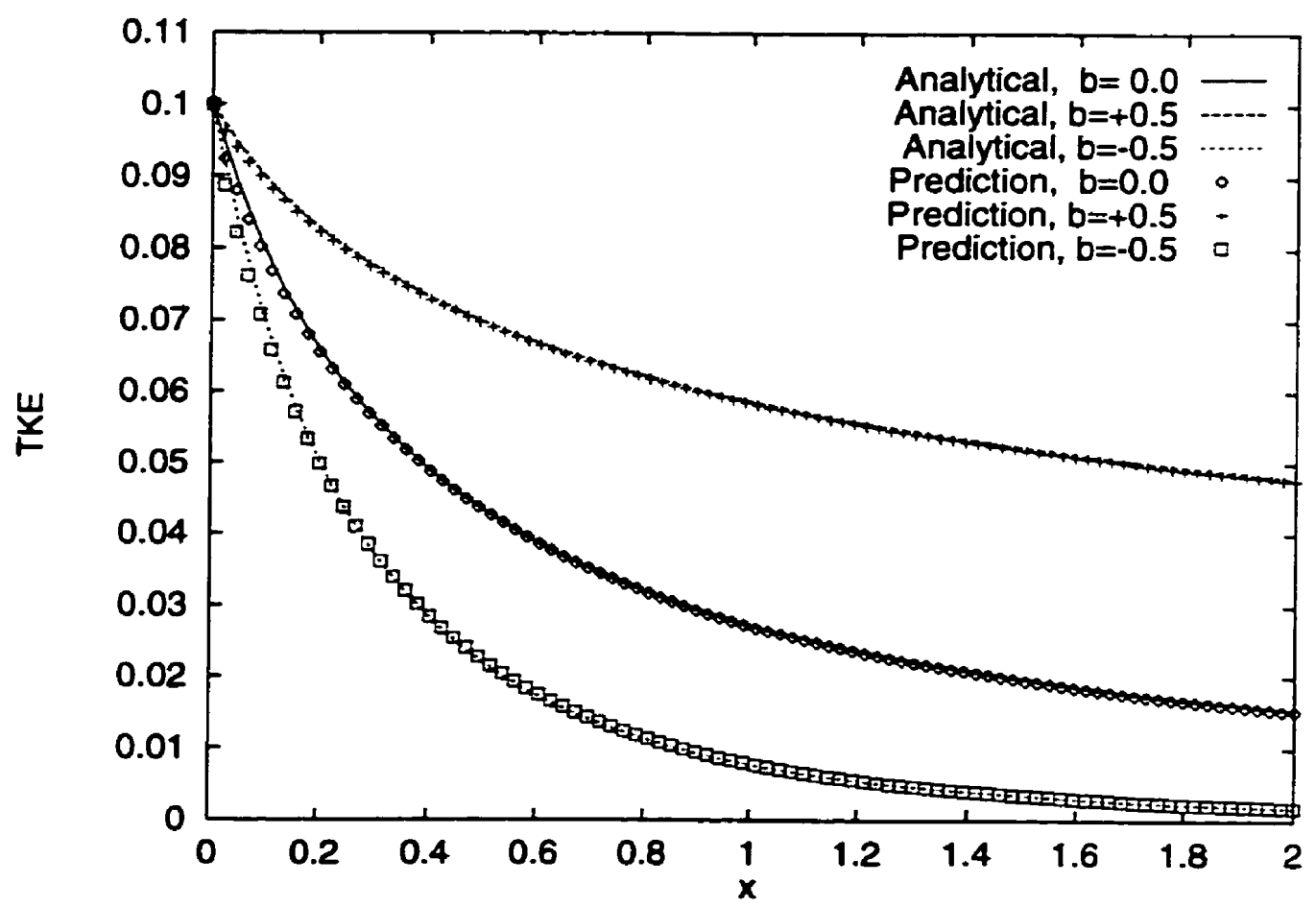

Figure 5.19: Analytical solution and code predictions for $k$ 
Recall from Section 4.4 that the average volumetric source term to the momentum equation due to the presence of the particles is given by

$$
F_{p}=\alpha\left(u_{g}-u_{p o}\right)\left(1-\exp \left(-\frac{\delta t}{\tau_{p}}\right)\right)
$$

where $u_{p o}$ is the initial particle velocity; $\alpha$ is the concentration of the particles per volume of flow, given by $\alpha=\dot{m}_{p} \delta t / F$, and $\delta t$ is the residence time of a particle. The arerage momentum source term, $\overline{F_{p}}$, can be obtained by dividing $F_{p}$ by the residence time of the particle, $\delta t$ and multiplying by the total volume of the flow domain such that.

$$
\overline{F_{p}}=\dot{m}_{p}\left(u_{g}-u_{p o}\right)\left(1-\exp \left(-\frac{\delta t}{\tau_{p}}\right)\right)
$$

The residence time of the particle $\delta t$ can be determined from integrating the particle equation of motion, Eq. (2.7), over the interval from $t=0$ to $t=\delta t$. Neglecting gravity: this yields:

$$
x_{p}=u_{g} \delta t-\tau_{p}\left(\exp \left(-\frac{\delta t}{\tau_{p}}\right)-1\right)\left(u_{p o}-u_{g}\right)
$$

where $x_{p}$ is the distance traveled by the particle, in this case, the length of the pipe.

The value of the average momentum source term obtained analytically exactly matched the value given in the output from TASCflow.

\subsection{Summary}

This chapter outlined the validations performed to ensure that TASCflow could correctlyimplement the turbulence modulation models and to gain insight into the accuracy of the predictions.

The laminar pipe flow simulation showed that a wedge-shaped grid can accuratelypredict flow through a circular pipe. Grid-independence was established and the sensitivity of the solution to inlet conditions, log-law of the wall and near-wall node placement examined. The ability of the code to predict particle dispersion was also established. Analytical worked yielded the interesting result that when modeling particle dispersion in a wedge-shaped grid the variance of the displacement normal to the direction of the mean flow, $\overline{x^{2}}$, is given by $\overline{r^{2}} / 2$. The analytical solution to the $k$ and $\epsilon$ equations for a grid generated turbulent flow were used to ensure that the turbulent source terms were implemented correctly. Finally, the implementation of momentum source terms was examined and confidence obtained in the solution. Such rigorous validation allows a better insight into the limitations intrinsic in TASCHow, the $k-\epsilon$ model and the SSF model. 
This provides a sense of the minimum error that can be expected when the turbulence modulation models are implemented. 


\section{Chapter 6}

\section{Results}

\subsection{Introduction}

Selected turbulence modulation models described in Chapter 2 are evaluated br comparing the model predictions to the experimental results of Tsuji et al [44]. The experiment involved an air-particle flow through a vertical pipe. The experiment was simulated using the TASCflow CFD code with the turbulence modulation models incorporated and the results obtained were compared to the experimental data.

The experiment of Tsuji et al is outlined. The turbulence modulation models used in this investigation are presented and briefly discussed and details of the simulation given. The results from the simulation are compared with the experimental data and the models' ability to predict general and experimental trends is discussed. An analysis is performed examining the behaviour of the models for various values of the particle time constant, $\tau_{p}$.

\subsection{The Experiment of Tsuji et al, 1983}

In the experiment of Tsuji et al [44] particles were released into a upward flowing vertical pipe. The use of a vertical pipe removed the asymmetrical effects of gravity from the flow. Referring to Figure 6.1, the particles were released into the flow and passed around a $90^{\circ}$ bend to enter the vertical test section as a two-phase flow. The pipe diameter changed abruptly at the bend outlet (from $40 \mathrm{~mm}$ to $30.5 \mathrm{~mm}$ ) causing the particles sliding on the outer wall of the bend to become dispersed. This ensured that the effect of the bend was not reflected in the particle distribution. The vertical test section consisted of a $30.5 \mathrm{~mm}$ diameter, $5.11 \mathrm{~m}$ long glass pipe. The length of the pipe was such that the flow was fully developed at the end test section. Simultaneous gas and particle relocity. measurements were taken using a laser-Doppler velocimeter (LDV) at the end of the test 


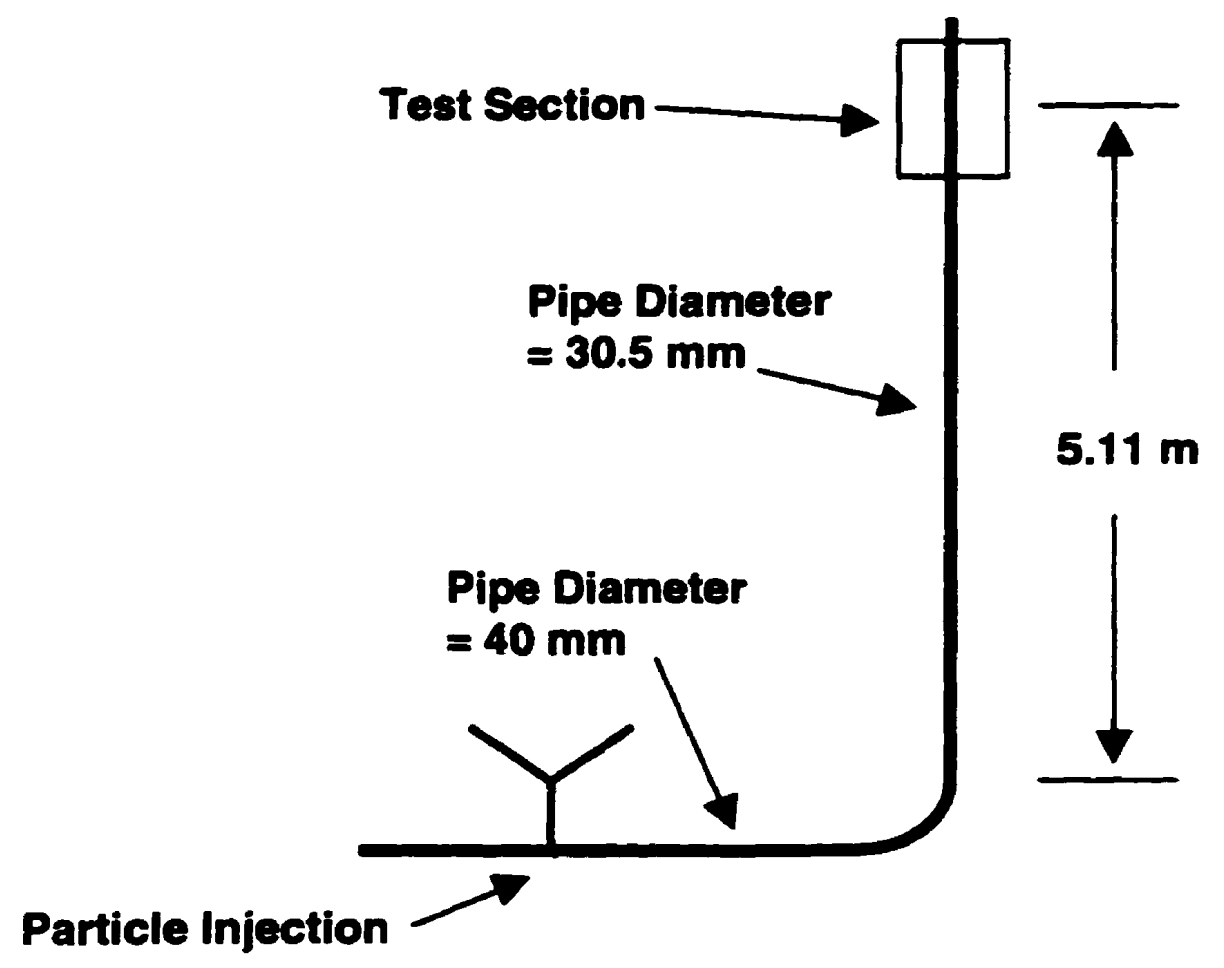

Figure 6.1: Experimental setup

section $(5.11 \mathrm{~m})$. This was achieved using a signal-discrimination device that is based on the principle that the burst signals with large pedestal components come from the large particles while burst signals with large Doppler but small pedestal components come form the tracer particles. Details are available in Tsuji and Morikawa (1982)[43] and Tsuji et al (1983)[44]. Radial turbulence intensity and mean velocity profiles were obtained at the end of the test section $(5.11 \mathrm{~m})$ in the fully developed region of the pipe.

This experiment used four kinds of spherical plastic pellets and one plastic cylinder. The polystyrene, spherical particles ranged in diameter from $200 \mu \mathrm{m}$ to $3 \mathrm{~mm}$ with densities from $1020 \mathrm{~kg} / \mathrm{m}^{3}$ to $1030 \mathrm{~kg} / \mathrm{m}^{3}$. Because electrostatic charging caused by friction between the particles and the pipe wall is significant for small particles, the 200 $\mu \mathrm{m}$ and $500 \mu \mathrm{m}$ particles were coated with a charge-preventing substance. The carricr phase was air with a density of $\rho_{g}=1.178$ and a viscosity of $1.84 * 10^{-05} \mathrm{Ns} / \mathrm{m}^{2}$. Mean air velocities range from 8 to $20 \mathrm{~m} / \mathrm{s}$ and particle loading ranges are up to 5 . 
Table 6.1: Turbulence Modulation Models to be Investigated

\begin{tabular}{|c|c|c|c|}
\hline Model & $\overline{S_{k}}$ & $\overline{S_{\epsilon}}$ & Comments \\
\hline $\begin{array}{l}\text { Chen \& } \\
\text { Wood }\end{array}$ & $\frac{2 k \alpha}{\rho_{g} \tau_{p}}\left[1-\exp \left(\frac{B_{k} T_{p} \epsilon}{0.165 k}\right)\right]$ & $-\frac{2 \alpha}{\rho_{g} T_{p}} \epsilon$ & $B_{k}=0.0825$ \\
\hline $\begin{array}{l}\text { Tu \& } \\
\text { Fletcher }\end{array}$ & $\frac{2 k \alpha}{\rho_{g} \tau_{p}}\left[1-\exp \left(-\frac{0.09 \tau_{p} \epsilon}{w^{n} k}\right)\right]$ & $-\frac{2 \epsilon \alpha}{\rho_{g} \tau_{p}}\left[1-\exp \left(-\frac{0.4 \tau_{p} \epsilon}{w^{n} k}\right)\right]$ & $\begin{array}{l}n=0, w \leq 1 \\
n=1 . w>1\end{array}$ \\
\hline $\begin{array}{l}\text { Mostafa \& } \\
\text { Mongia }\end{array}$ & $\frac{2 k a}{\rho_{g} \tau_{p}}\left(1-\frac{\tau_{L L}}{\tau_{L I}+\tau_{p}}\right)$ & $-C_{\epsilon 3} \frac{2 \epsilon a}{p_{g} \tau_{p}}\left(1-\frac{\pi}{\tau_{l}+\tau_{p}}\right)$ & $\begin{array}{c}\tau_{l}=0.35 \mathrm{k} / \epsilon \\
C_{\epsilon 3}=1.00\end{array}$ \\
\hline $\begin{array}{l}\text { Lightstone } \\
\text { \& Hodgson }\end{array}$ & $\frac{2 k \alpha}{\rho_{g} \tau_{p}}\left(1-\frac{\tau^{\cdot}}{\tau^{*}+\tau_{p}}\right)$ & $-\frac{2 \epsilon \alpha}{\rho_{g} \tau_{p}}\left(1-\frac{\tau^{*}}{\tau^{+} \tau_{p}}\right)$ & $\begin{array}{l}\frac{1}{\tau^{*}}=\frac{u_{R E L}}{L_{I}}+\frac{i}{\tau_{L I}} \\
\tau_{L I}=0.13 .5 k / \epsilon \\
L_{I}=2 \tau_{L I} \sqrt{2 k / 3}\end{array}$ \\
\hline
\end{tabular}

\subsection{Turbulence Modulation Models}

Recall from Section 2.3.4 that the turbulence modulation models appear as sources in the $k$ and $\epsilon$ equations. The exact expression for the source terms are repeated below:

$$
\begin{aligned}
S_{k} & =\frac{1}{\rho_{g}} \frac{\alpha}{\tau_{p}}\left(\overline{u_{g i}^{\prime} u_{g i}^{\prime}}-\overline{u_{g i}^{\prime} u_{p i}^{\prime}}\right) \\
S_{\epsilon} & =\frac{1}{\rho_{g}} \frac{2}{\tau_{p}} \alpha \overline{\left.\nu \frac{\partial u_{g i}^{\prime}}{\partial x_{j}}\left(\frac{\partial u_{g i}^{\prime}}{\partial x_{j}}-\frac{\partial u_{p i}^{\prime}}{\partial x_{j}}\right)\right]}
\end{aligned}
$$

Note that $S_{k}$ appears as a sink to the $k$ equation while $S_{\epsilon}$ appears as a source to the $\epsilon$ equation. Four turbulence modulation models were selected for this investigation: Chen and Wood [4], Tu and Fletcher [45], Mostafa and Mongia [30] and Lightstone and Hodgson. These models were presented in Section 2.3.4 and are summarized in Table 6.1 .

These models were selected for use in this investigation because they are easily implemented as sources in the $k-\epsilon$ model and are compatible with the implementation procedure used in TASCflow. 
In order to implement the model of Lightstone and Hodgson in TASCflow, a modification to the model was required. Because of the way TASCflow is designed, the relative velocity field, $u_{R E L}$, is not available to the portion of the code where the model is implemented. Therefore, it was assumed that the particles were at their terminal velocity in the test section and the approximation $u_{R E L}=\tau_{p} g$ was made.

\subsection{Simulation}

The 45000 node wedge-shaped grid described in Chapter 5 was used in this investigation. The grid was long enough to ensure fully developed flow at the outlet, where all velocity and turbulence intensity radial profiles were taken. The initial velocities of the gasphase were not reported in the literature so the velocity profiles for each test case were integrated to obtain an average velocity used as the inlet velocity. The inlet velocity was specified as constant across the inlet plane. The inlet turbulence intensity and eddylength scale were set to 0.06 and $9.15^{*} 10^{-} 4$ respectively. The static pressure was set to 0 at one face on the outlet plane.

The total number of particles injected was 1500 . These particles were injected with a constant mass flux across the inlet plane. The mass flux of the particles was not reported in the literature; it was therefore calculated from the loading ratio, $w$, such that:

$$
\dot{m}_{p}=w \rho_{g} U_{g} A_{\text {wedge }}
$$

The initial velocity of the particles was set equal to the gas-phase, $u_{R E L}(0)=0$. The source terms were implemented into TASCflow according to the procedure outlined in Section 4.4.

This investigation will focus on the $200 \mu \mathrm{m}$ and $500 \mu \mathrm{m}$ diameter particles. Three test cases were selected and are presented in Table 6.2. Case 1 involved the $200 \mu \mathrm{m}$ diameter particles at a loading ratio of 3.2. Case 2 involved the the $200 \mu \mathrm{m}$ diameter particles at a loading ratio of 1.3 . Case 3 involved the $500 \mu \mathrm{m}$ diameter particles at a loading ratio of 3.4. This provided a measure of the turbulence modulation models' ability to predict trends due to a change in particle size (Case 1 vs. Case 3) and loading ratio (Case 1 r's. Case 2).

\subsection{Results}

In this section the results of the simulations are presented. First, each case is looked at in turn; the results from the models are compared against each other and the re- 
Table 6.2: Particle test cases

\begin{tabular}{|c|c|c|c|}
\hline & Case 1 & Case 2 & Case 3 \\
\hline$d_{p}$ & $200 \mu \mathrm{m}$ & $200 \mu \mathrm{m}$ & $500 \mu \mathrm{m}$ \\
$w$ & 3.2 & 1.3 & 3.4 \\
$\rho_{p}$ & $1020 \mathrm{~kg} / \mathrm{m}^{3}$ & $1020 \mathrm{~kg} / \mathrm{m}^{3}$ & $1020 \mathrm{~kg} / \mathrm{m}^{3}$ \\
$U_{g}$ & $9.62 \mathrm{~m} / \mathrm{s}$ & $10.5 \mathrm{~m} / \mathrm{s}$ & $9.66 \mathrm{~m} / \mathrm{s}$ \\
$\dot{m}_{p}$ & $1.104^{*} 10^{-} 3 \mathrm{~kg} / \mathrm{s}$ & $4.89^{*} 10^{-4} \mathrm{~kg} / \mathrm{s}$ & $1.176^{*} 10^{-} 3 \mathrm{~kg} / \mathrm{s}$ \\
\hline
\end{tabular}

sults discussed. Then the results from each model are looked at, in order to gain an understanding of each model's ability to predict the experimental trends.

The experimental data reports turbulence intensity and mean velocity data for a clean (particle-free) run. It is important to note that this clean run and each subsequent run with a change in loading ratio within the size group have different mass flows from one another. Thus, the clean data presented on each plot is from the simulation: the ability of TASCflow to predict Tsuji et al's particle-free experimental results was discussed in Section 5.2.

\subsubsection{Case 1}

The results from the simulations are shown in Figures 6.2 and 6.3. As a basis for comparison, the analytical solution for a laminar flow at the same mass flow rate as the turbulent flows is plotted against the results.

To verify that the solution obtained using 1500 particles was statistically significant the number of particles injected was doubled to 3000 , and the solution re-run using the model of Chen and Wood. Figure 6.4 shows that the turbulence intensity solution exhibits little change with the injection of 3000 particles. It is therefore concluded that the solution obtained with 1500 particles is statistically significant.

Looking at the velocity profiles, those predicted by the models of Chen and Wood. Mostafa and Mongia, and Lightstone and Hodgson all predict an increase in the steepness of the velocity profiles, while the experimental results suggest that the velocity profile should become flatter with the introduction of particles. To verify that the solution obtained from the models made physical sense, the turbulent viscosity, $\mu_{t}$, was plotted 


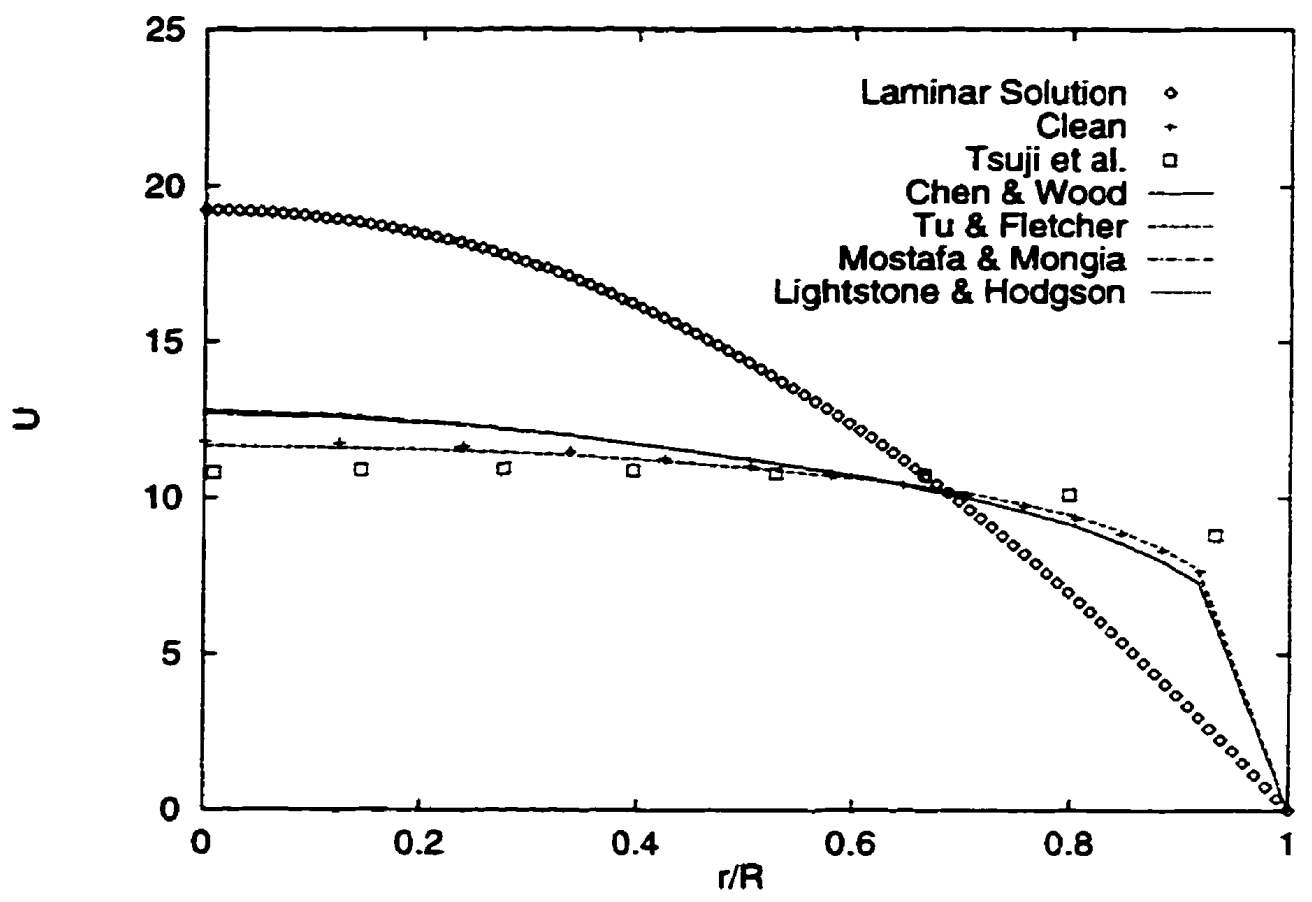

Figure 6.2: Mean velocity: Predictions of experimental data of Tsuji et al[ $[1-1]$. $R e \approx$ $23000, d_{p}=200 \mu \mathrm{m}, w=3.2$ (Case 1 )

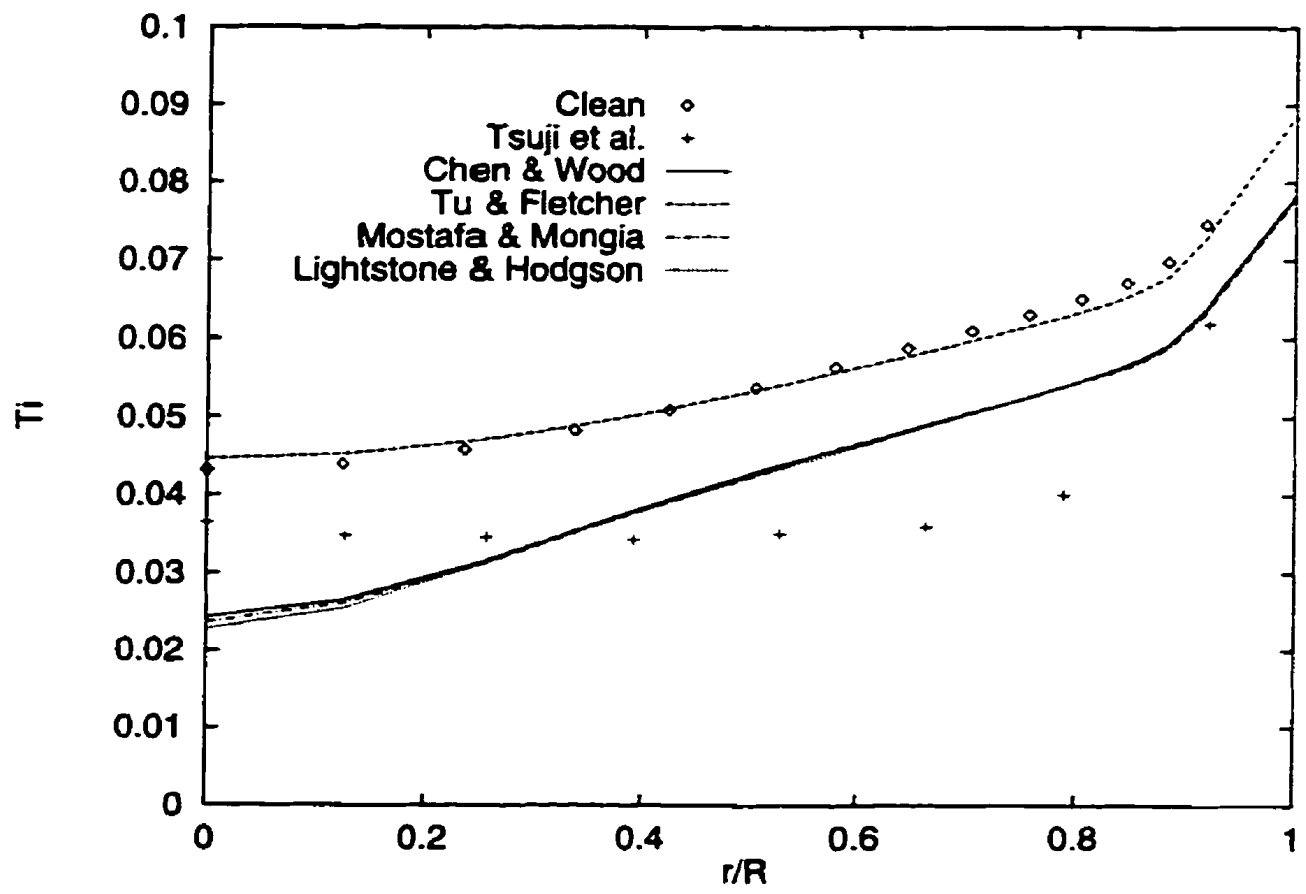

Figure 6.3: Turbulence intensity: Predictions of experimental data of Tsuji et al[ $4 t]$. $R e \approx 23000, d_{p}=200 \mu \mathrm{m}, w=3.2$ (Case 1 ) 


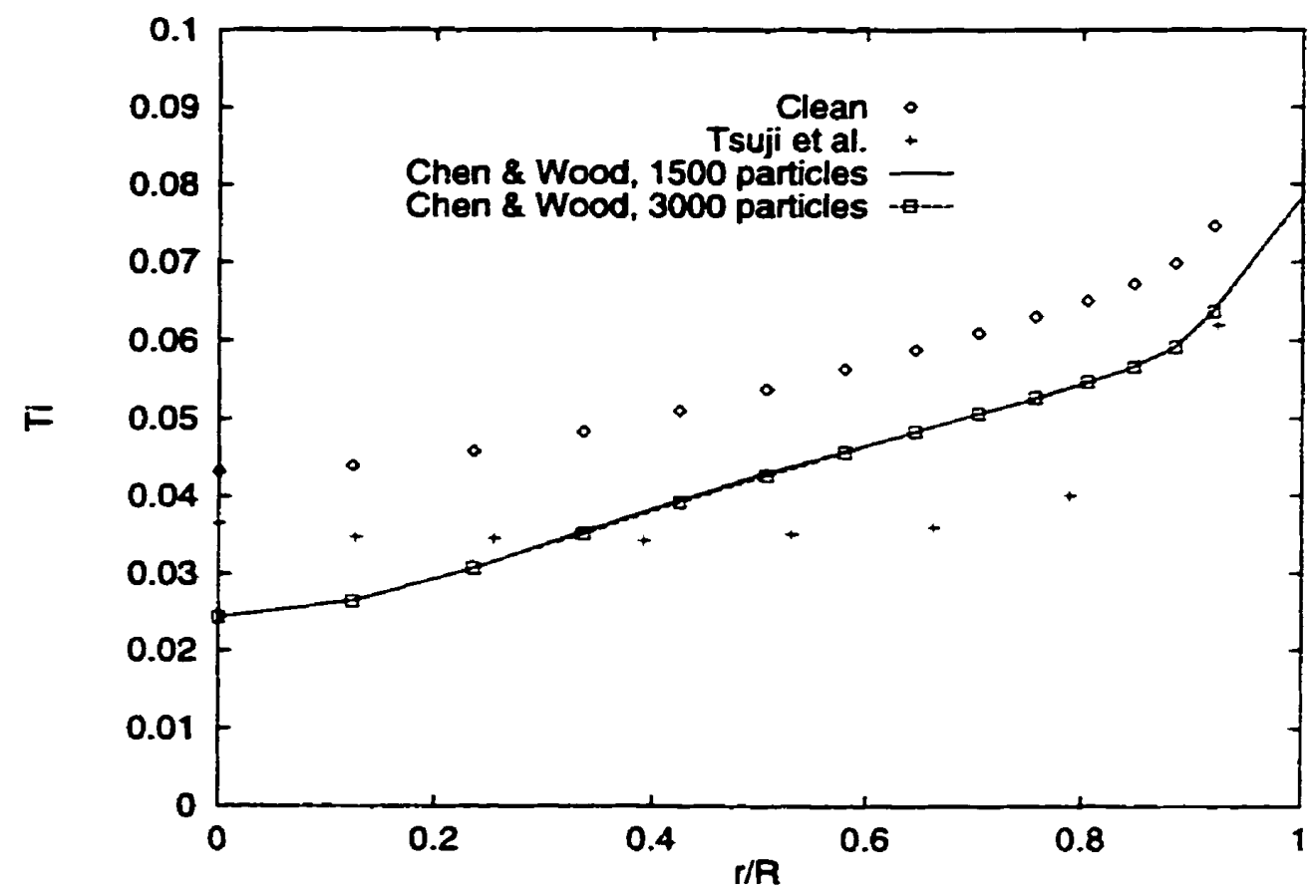

Figure 6.4: Effect of doubling number of particles injected: Turbulence intensity predictions of experimental data of Tsuji et al[44], $R e \approx 23000, d_{p}=200 \mu \mathrm{m}, w=3.2$ (Cassc 1)

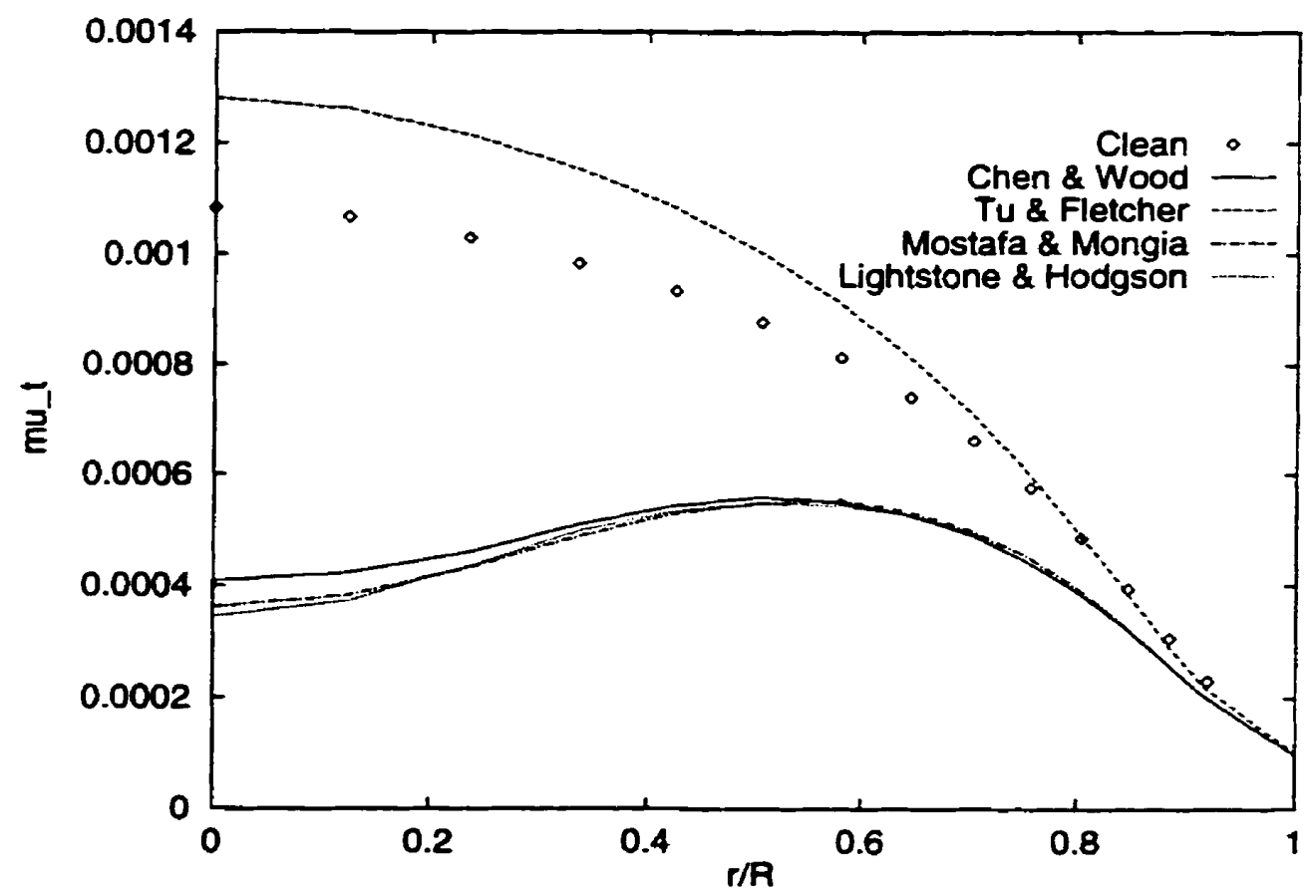

Figure 6.5: Turbulent Viscosity, $\mu_{t}$ : Predictions from the models and clean solution. Case 1. 
for both the clean flow and the particle-laden flow. If the change in velocity profile is physically correct, there should be a decrease in $\mu_{t}$ for these three models. Figure 6.5 shows that this is indeed the case. Likewise there should little change in $\mu_{t}$ for the prediction from the model of Tu and Fletcher. It is observed that there is in fact a small increase in $\mu_{t}$ for this model.

The models of Chen and Wood, Mostafa and Mongia and Lightstone and Hodgson give nearly identical predictions for turbulence intensity; near the centerline $T_{i}$ was underpredicted (to $r / R \approx 0.3$ ) and over-predicted in the rest of the pipe. The model of Tu and Fletcher predicts very little change from the clean solution for the turbulence intensity profile. In order to determine why the results of Chen and Wood, Mostafa and Mongia. and Lightstone and Hodgson are nearly identical and differ from those of Tu and Fletcher. numerical analysis was performed.

Looking at Table 6.1, all of the models are in the form:

$$
S_{k}=\frac{2 k \alpha}{\rho_{g} \tau_{p}} f
$$

where $f$ is a dimensionless function.

The value of $f$ appearing in Equation (6.4) was calculated using centerline values of $k, \epsilon, \alpha$ taken from Chen and Wood's solution. The models of Chen and Wood. Mostafa and Mongia, and Lightstone and Hodgson all give results of 90 to $100 \%$ of $(2 k \alpha) /\left(\tau_{p} \rho_{g}\right)$ while the model of $\mathrm{Tu}$ and Fletcher gives the significantly different result of $10 \%$. This accounts for the different prediction given by the model of $\mathrm{Tu}$ and Fletcher from the others. The values obtained for $f$ are presented in Table 6.3.

Next, in order to determine how the models interact with the $k-\epsilon$ model. the expression for the turbulent kinetic energy, $k$, was broken down into several components. Recall from Section 2.2.3 that the expression for $k$ is composed of the following processes:

$$
\rho_{g} \frac{D k}{D t}=\text { Diffusion }+ \text { Production }- \text { Dissipation }-\rho_{g} S_{k}
$$

Plots of the Production, Dissipation and source term due to particle-turbulence interaction, $S_{k}$, are shown in Figures 6.6 to 6.8. It is clear from these plots that, as expected. the results obtained using the model of Tu and Fletcher differ significantly from the other three models. However, Figure 6.9 shows that for the total source to the $k$ equation. that is Production - Dissipation - $S_{k}$, the results from Tu and Fletcher's model are indistinguishable from the results of the other models. This is an interesting result. If the significant difference in the models for the turbulence intensity profile is not a result of the total source to the $k$-equation then it must be through the diffusion term that the 
Table 6.3: Summary of Handchecks: Case 1

\begin{tabular}{|c|c|}
\hline Model & +2 \\
\hline Chen \& Wood & $\left(1-\exp \left(\frac{-0.5 \tau_{p} \epsilon}{k}\right)\right)=0.9$ \\
\hline Tu \& Fletcher & $\left(1-\exp \left(\frac{-0.09 \tau_{\rho} \epsilon}{3.2 k}\right)\right)=0.1$ \\
\hline Mostafa \& Mongia & $\left(1-\frac{\pi}{\eta_{n}+\tau_{p}}\right)=$ \\
\hline Lightstone \& Hodgson & $\left(1-\frac{\tau^{*}}{\tau^{*}+\tau_{0}}\right)=$ \\
\hline
\end{tabular}

turbulent kinetic energy profiles are modified.

The diffusion term appearing in the $k-\epsilon$ model is of the form:

$$
\text { Diffusion }=\frac{\partial}{\partial x_{k}}\left[\frac{\mu_{t}}{\sigma_{k}} \frac{\partial k}{\partial x_{k}}\right]
$$

where $\sigma_{k}=1$.

The diffusion term will act to 'smear' the turbulence intensity profiles. The higher the value of $\mu_{t}$ the flatter the turbulence intensity profile. Figure 6.5 shows the radial profiles for $\mu_{t}$ obtained at the outlet. The turbulent viscosity obtained at the centerline using the model of $\mathrm{Tu}$ and Fletcher is higher than that of both the clean flow and the turbulent viscosity obtained with the other three models. Thus, it is expected that the turbulence intensity profile obtained using $\mathrm{Tu}$ and Fletcher's model will be flatter than both the clean profile and the profiles obtained with the other three models. Looking at Figure 6.3 it is clear that this is the case. Therefore, it is concluded that the diffusion term, specifically $\mu_{t}$, plays an important role in determining the turbulence intensity across the pipe.

\subsubsection{Case 2}

This case once again involves particles with diameters of $200 \mu \mathrm{m}$, however the loading ratio for this case is 1.3 , less than half of the loading ratio of 3.2 used in Case 1. The results from the simulations are shown in Figures 6.10 and 6.11. The velocity profiles 


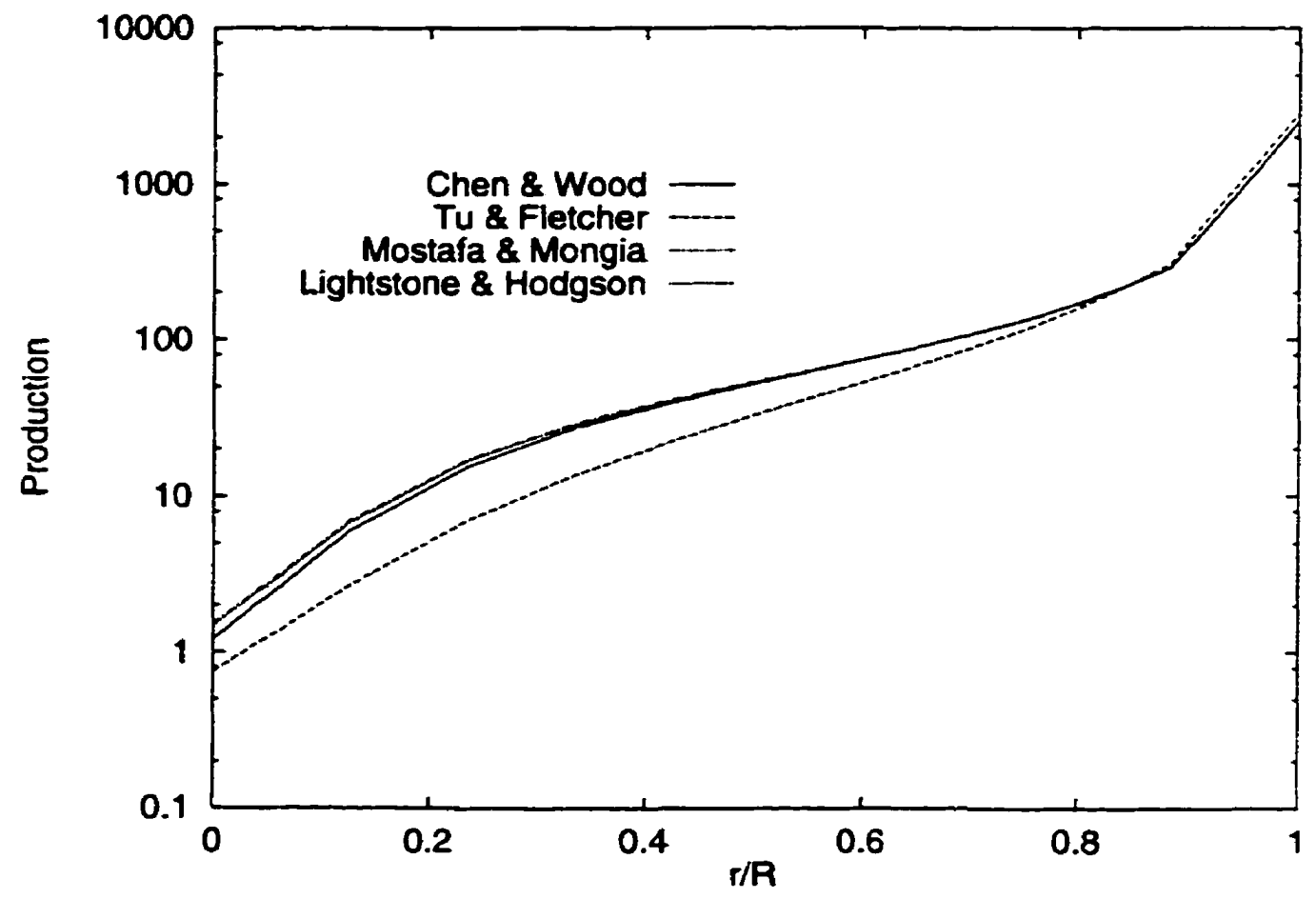

Figure 6.6: Production of $k$ : Case 1

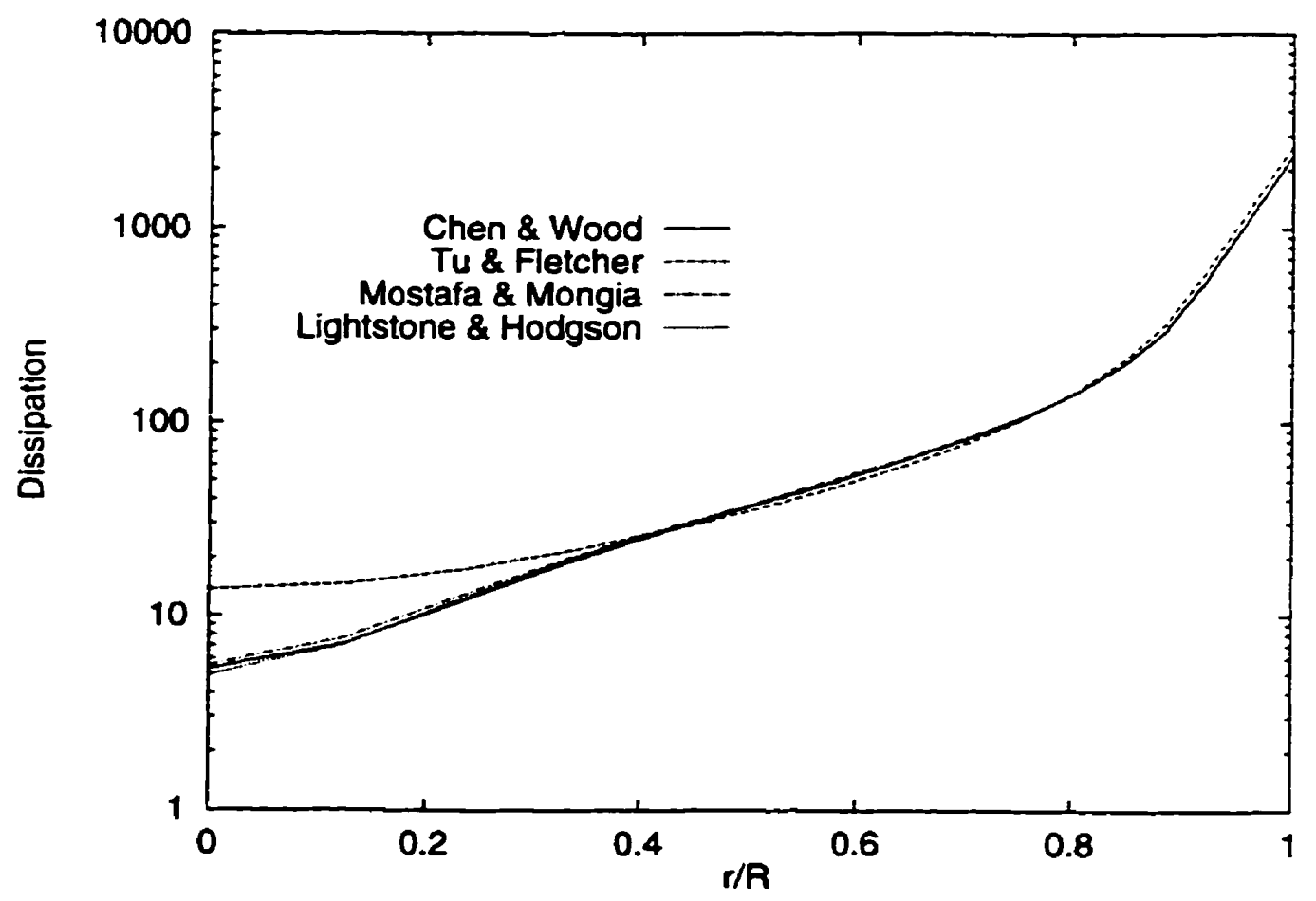

Figure 6.7: Dissipation of $k$ : Case 1 
Chapter 6. Results

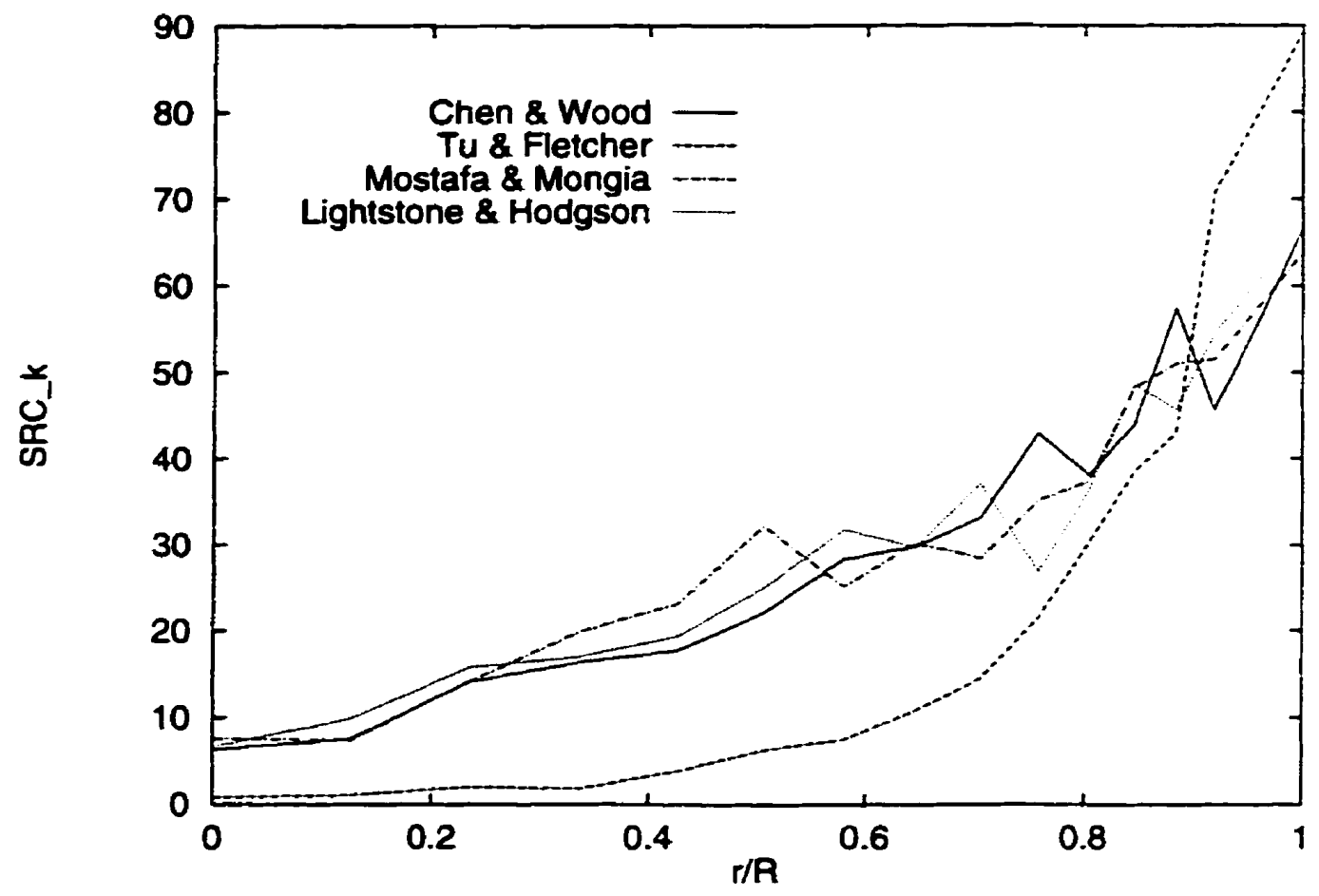

Figure 6.8: Source to $k$ equation, $S_{k}$ : Case 1

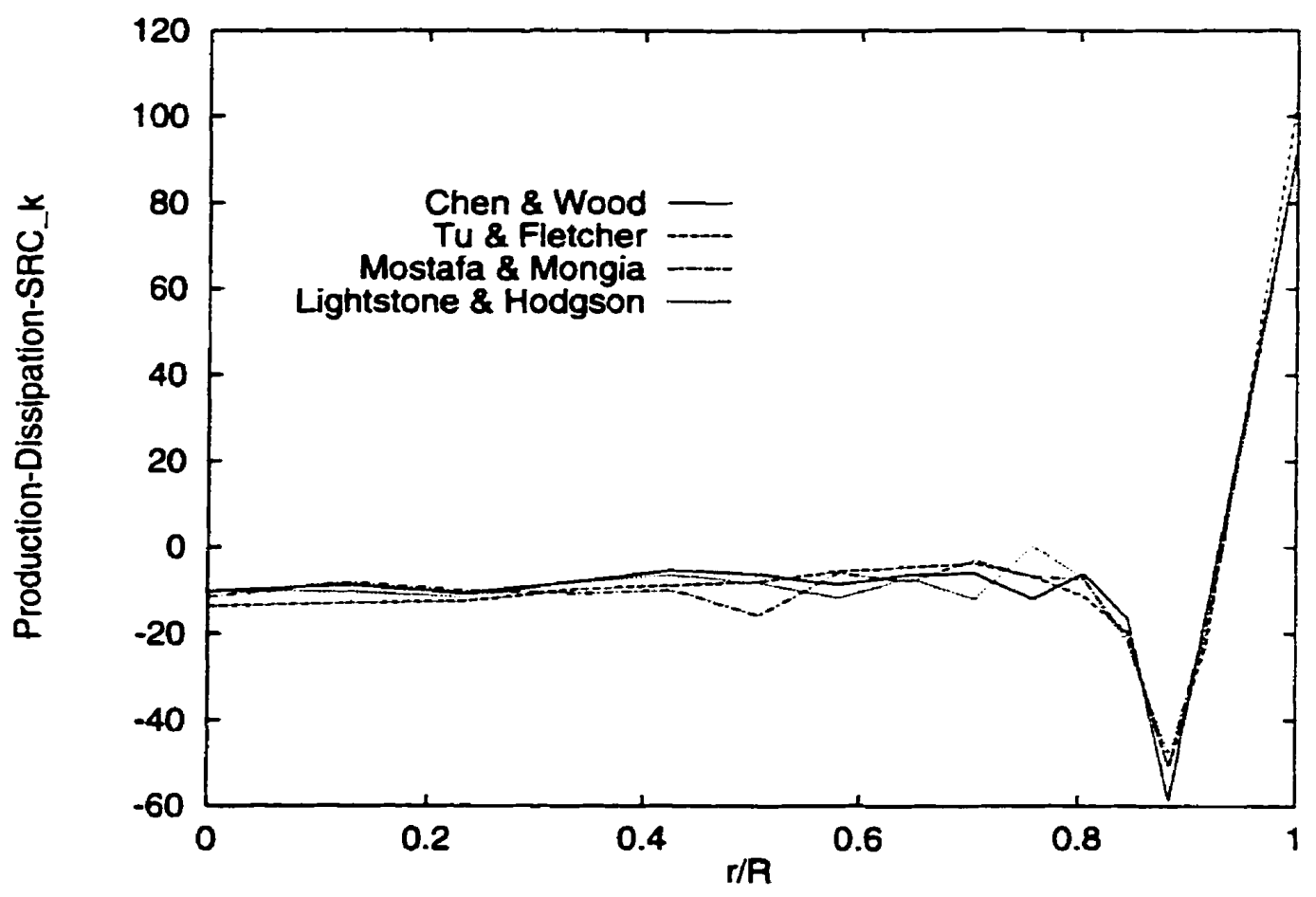

Figure 6.9: Total source to $k$ equation, Production $-\epsilon-S_{k}$ : Case 1 
predicted by all of the models are in good agreement with the experimental results. The turbulence intensity profiles are over-predicted by all of the models, with the model of Tu and Fletcher once again giving a different result than the other models.

Figures 6.12 to 6.15 show plots of production, dissipation, $S_{k}$, and the total source to the $k$ equation. The same trends exist for this case as were seen in Case 1 . There is a small difference between the production, dissipation and $S_{k}$ obtained using $\mathrm{Tu}$ and Fletcher's model and the others, however when the total source to the $k$ equation is examined, the result of $\mathrm{Tu}$ and Fletcher does not distinguish itself.

Figure 6.16 shows the turbulent viscosity profiles for this case. The turbulence viscosity profile of Tu and Fletcher is steeper than that of the other models; that is, the value of $\mu_{t}$ is higher across most of the pipe. This corresponds to the flatter turbulence intensityprofile obtained from Tu and Eletcher's results. Likewise, the $\mu_{t}$ for $\mathrm{Tu}$ and Fletcher is lower than the clean value across the pipe and a steeper turbulence intensity profile is seen. So as seen in Case 1 , the effect of the additional source term is incorporated. in part, through the turbulent viscosity, $\mu_{t}$.

\subsubsection{Case 3}

This case involves a change in particle size, from the $200 \mu \mathrm{m}$ used in Case 1 to $500 \mu \mathrm{m}$. The loading ratio is close to that of Case 1 ; for this case a loading ratio of 3.4 is used. for Case $1 w=3.2$. The predictions are shown in Figures 6.17 and 6.18. The experimental results were under-predicted by all of the models; this as expected since none of the models are able to predict an increase in turbulence intensity. The model of $\mathrm{Tu}$ and Fletcher again yields a different solution than the other models. Figures 6.19 to 6.22 show the variation in production, dissipation, $S_{k}$ and the total source to the $k$ equation. For this case there is very little difference between the the results of the four models. Also notice that the values for $S_{k}$ are significantly smaller than those in the other two cases. This is due to the increase in the value of $\tau_{p}$ associated with the larger particles used in this case.

The turbulent viscosity profiles are shown in Figure 6.23. The profile of $\mu_{t}$ from the model of $\mathrm{Tu}$ and Fletcher lies in between the clean profile and the profile obtained with the other models. This is properly reflected in the turbulence intensity profiles and once again it is shown that $\mu_{t}$ has a significant effect on the turbulence intensity profiles. 


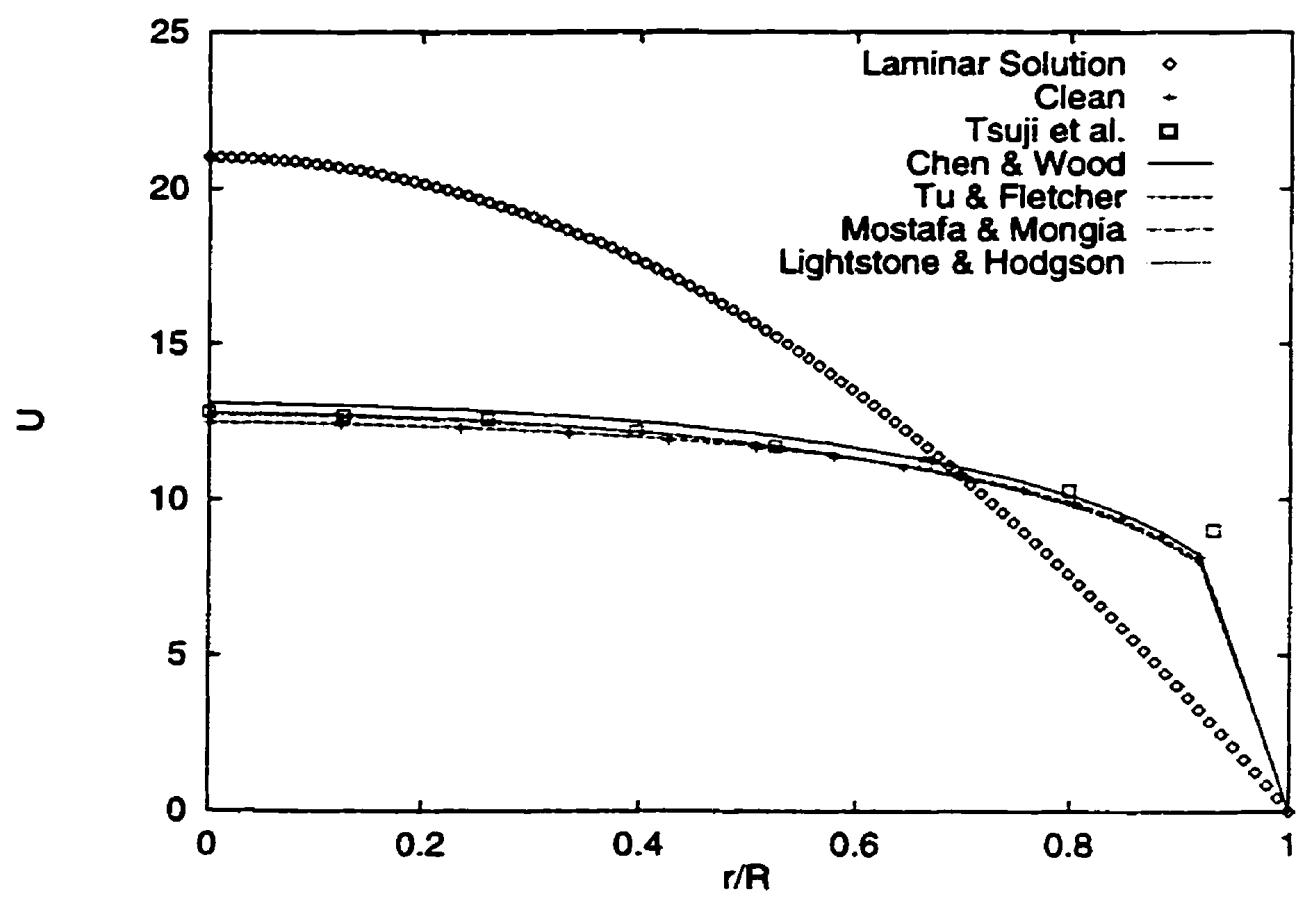

Figure 6.10: Mean velocity: Predictions of experimental data of Tsuji et al[44]. Re $\approx$ $23000, d_{p}=200 \mu \mathrm{m}, w=1.3$ (Case 2)

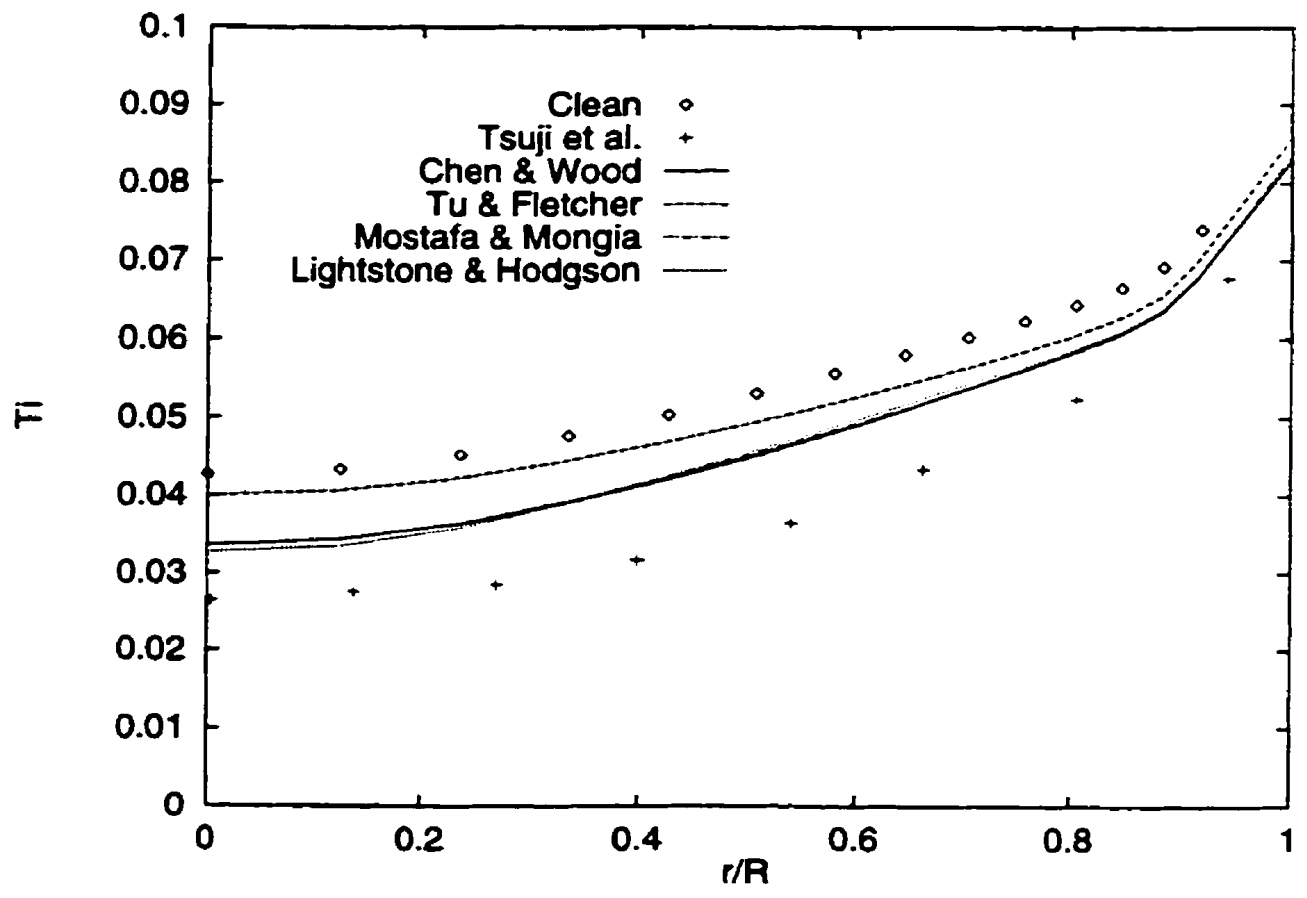

Figure 6.11: Turbulence intensity: Predictions of experimental data of Tsuji et al[ $t 4]$. $R e \approx 23000, d_{p}=200 \mu \mathrm{m}, w=1.3$ (Case 2) 


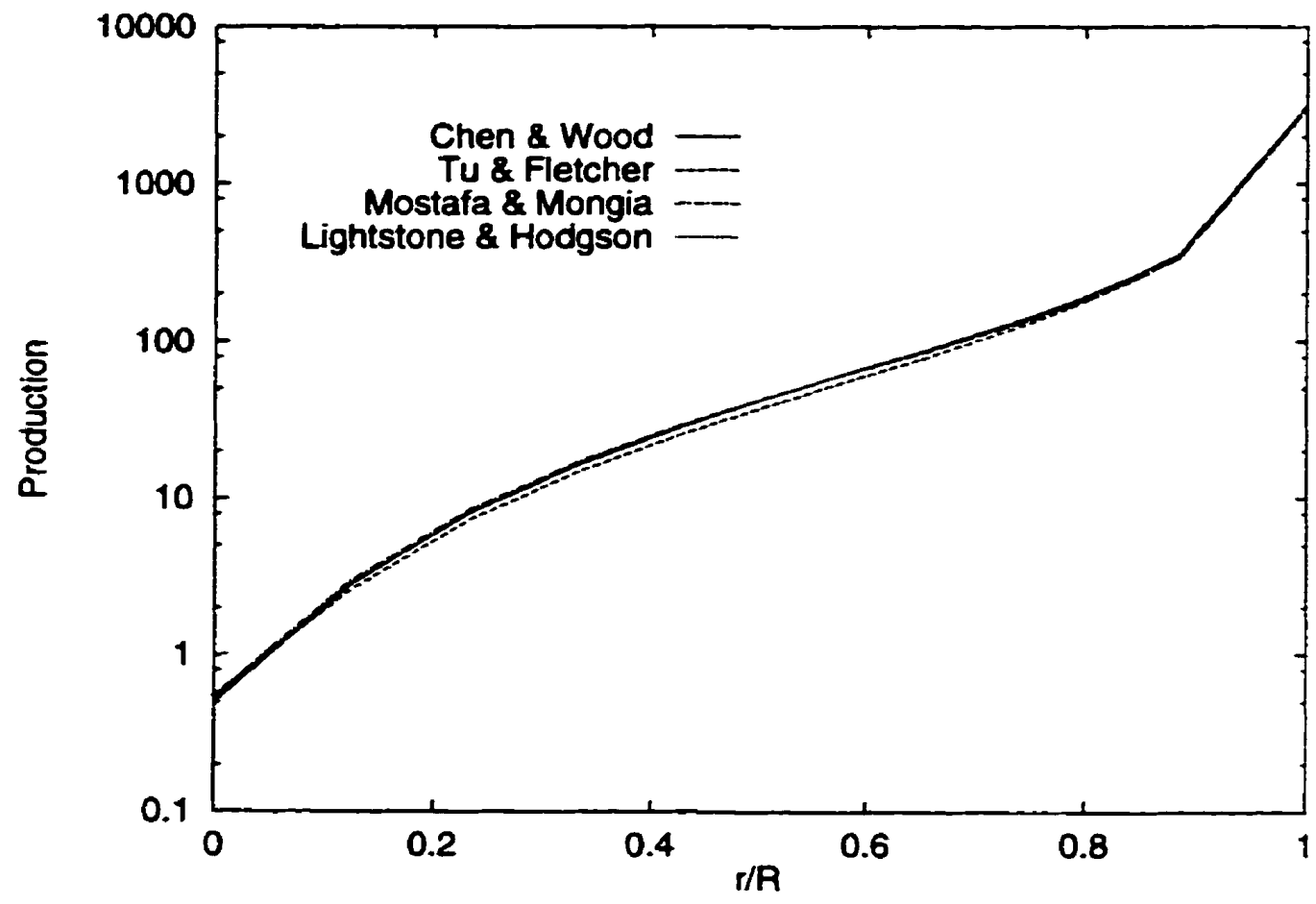

Figure 6.12: Production of $k$ : Case 2

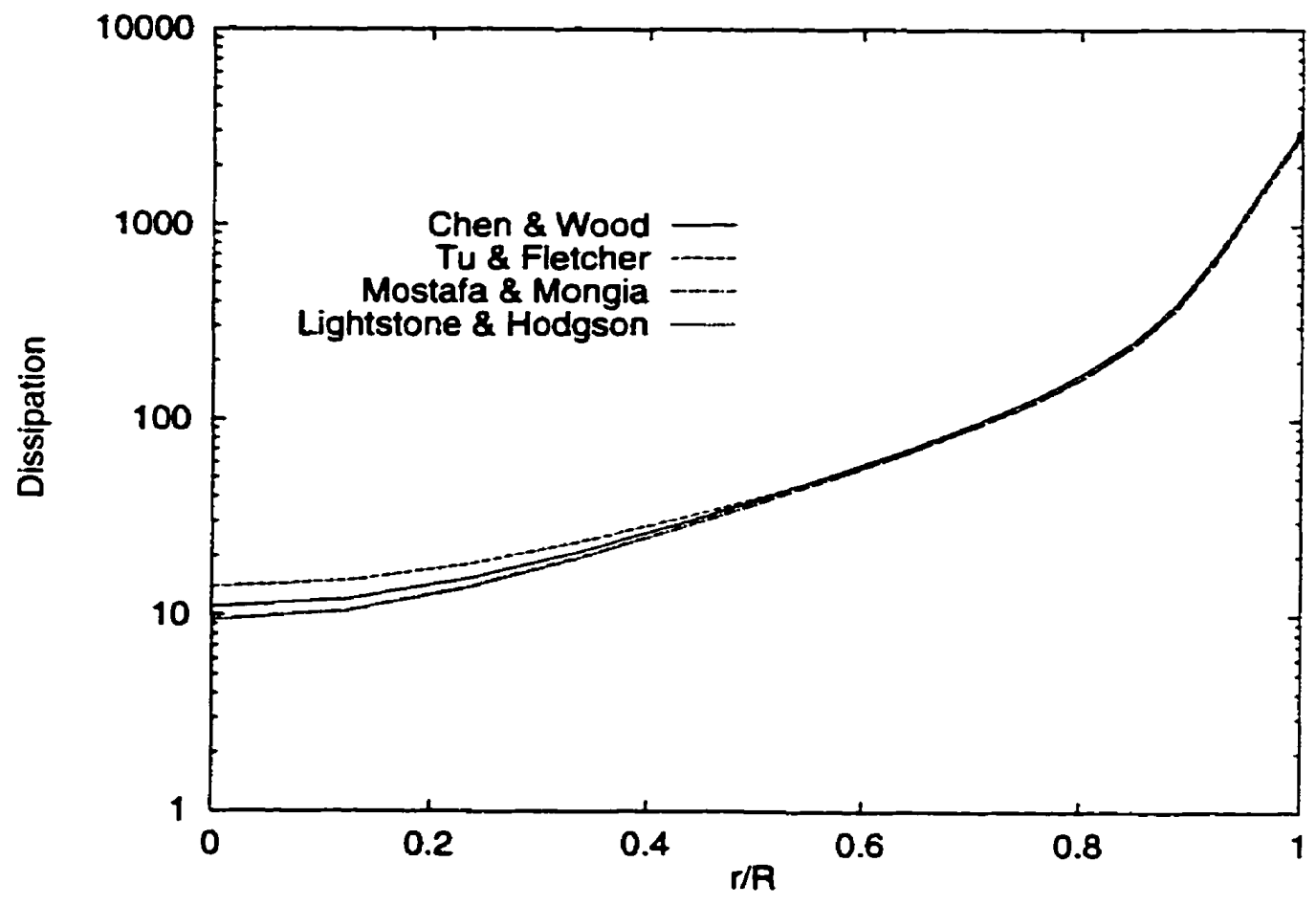

Figure 6.13: Dissipation of $k$ : Case 2 


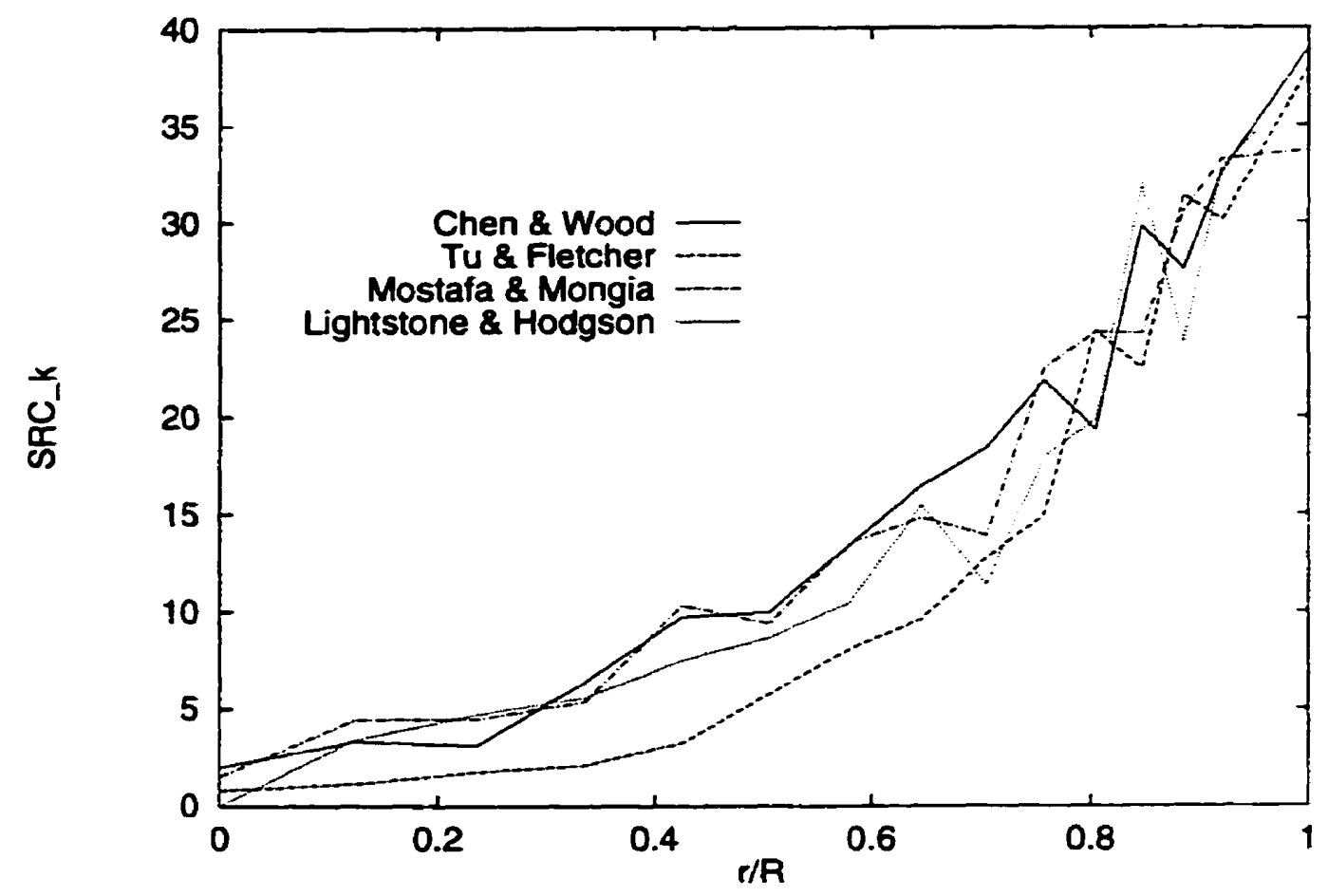

Figure 6.14: Source to $k$ equation, $S_{k}$ : Case 2

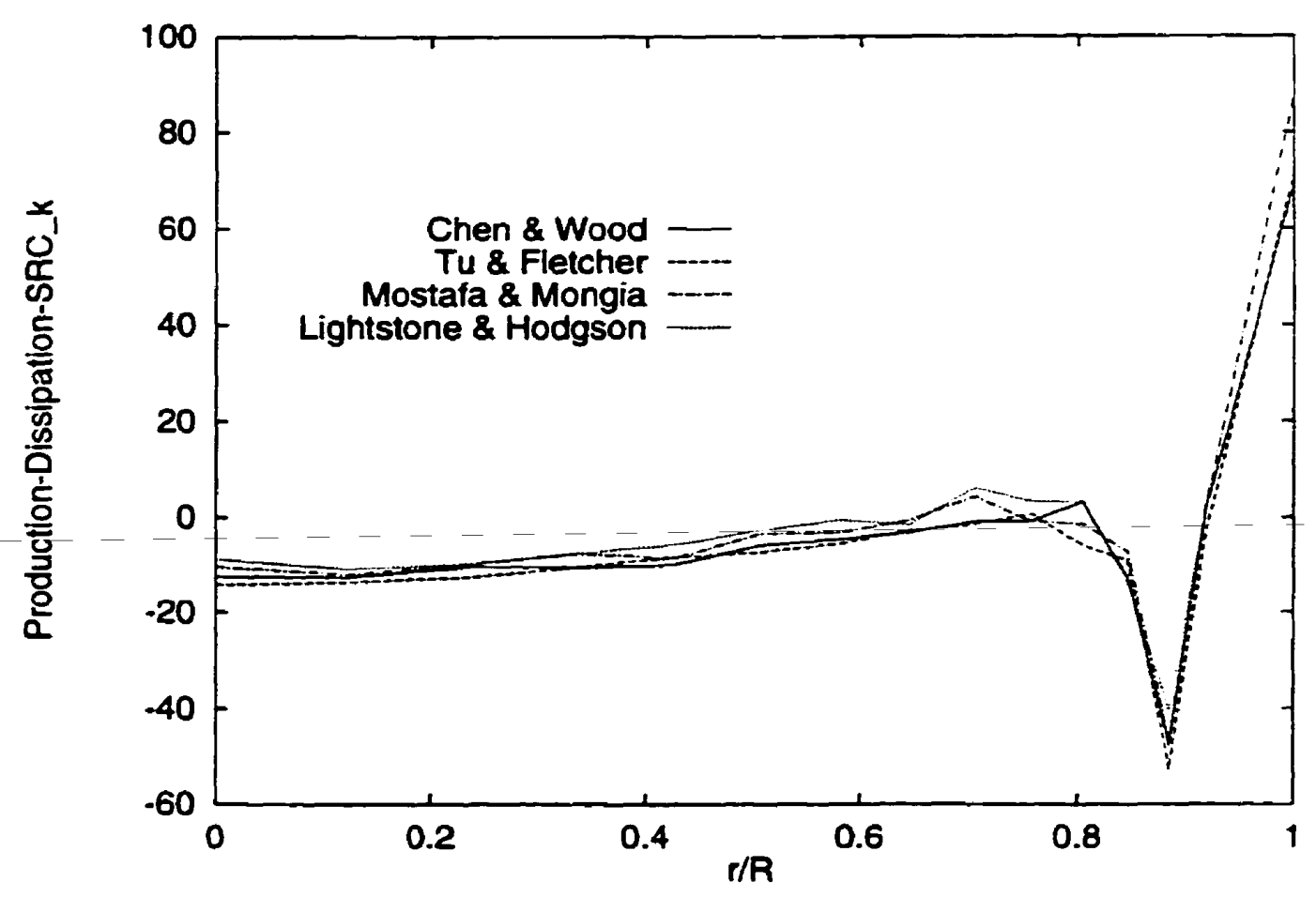

Figure 6.15: Total source to $k$ equation, Production $-\epsilon-S_{k}$ : Case 2 


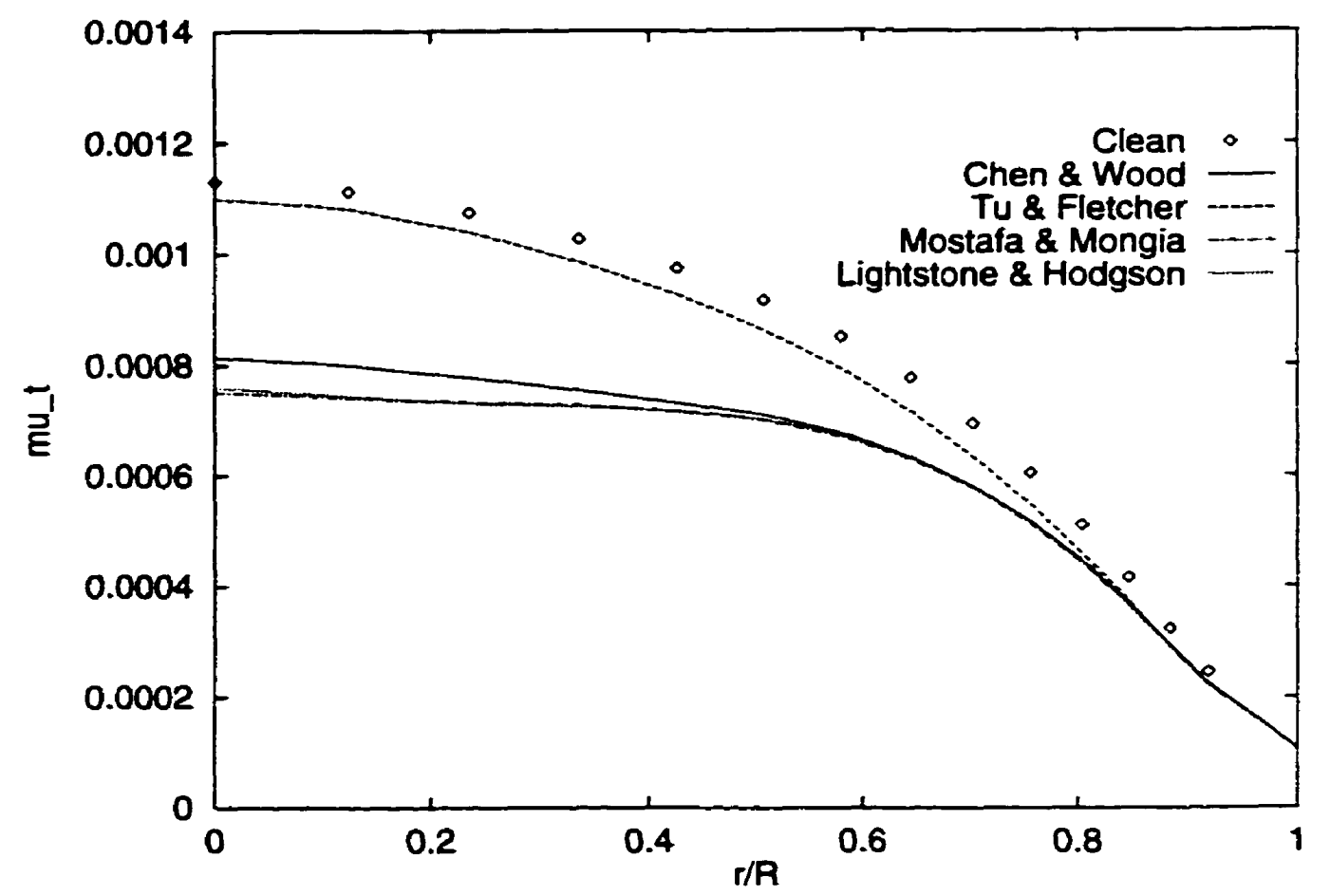

Figure 6.16: Turbulent Viscosity, $\mu_{t}$ : Predictions from the models and clean solution. Case 2.

\subsubsection{Turbulence Modulation Trend Predictions}

As discussed in Chapter 2, there are two main parameters which affect turbulence modulation: particle diameter and loading ratio. Recall that from experimental obserration there is an increase in turbulence intensity for large particles and a decrease in turbulence intensity for small particles. The loading ratio acts to further decrease the turbulence intensity for small particles and, conversely, further increase the turbulence intensity for large particles.

The three cases used for this investigation involve one change in particle diameter (200 $\mu \mathrm{m}$ to $500 \mu \mathrm{m}$ ) and one change in loading ratio ( $w=3.2$ to $w=1.3$ ). The expected result, according to the general experimental trend, is that an increase in turbulence intensity will be seen from Case 1 to Case 3, and Case 1 to Case 2 (reduction in loading ratio means a reduction in the magnitude of the decrease).

Figures 6.24 to 6.27 show the percent change in turbulence intensity predicted by the models. The percent change was calculated as:

$$
\text { \%Change in } T_{i}=\frac{T_{i, \text { Case } 2,3}-T_{i, \text { Case } 1}}{T_{i, \text { Case } 1}}
$$




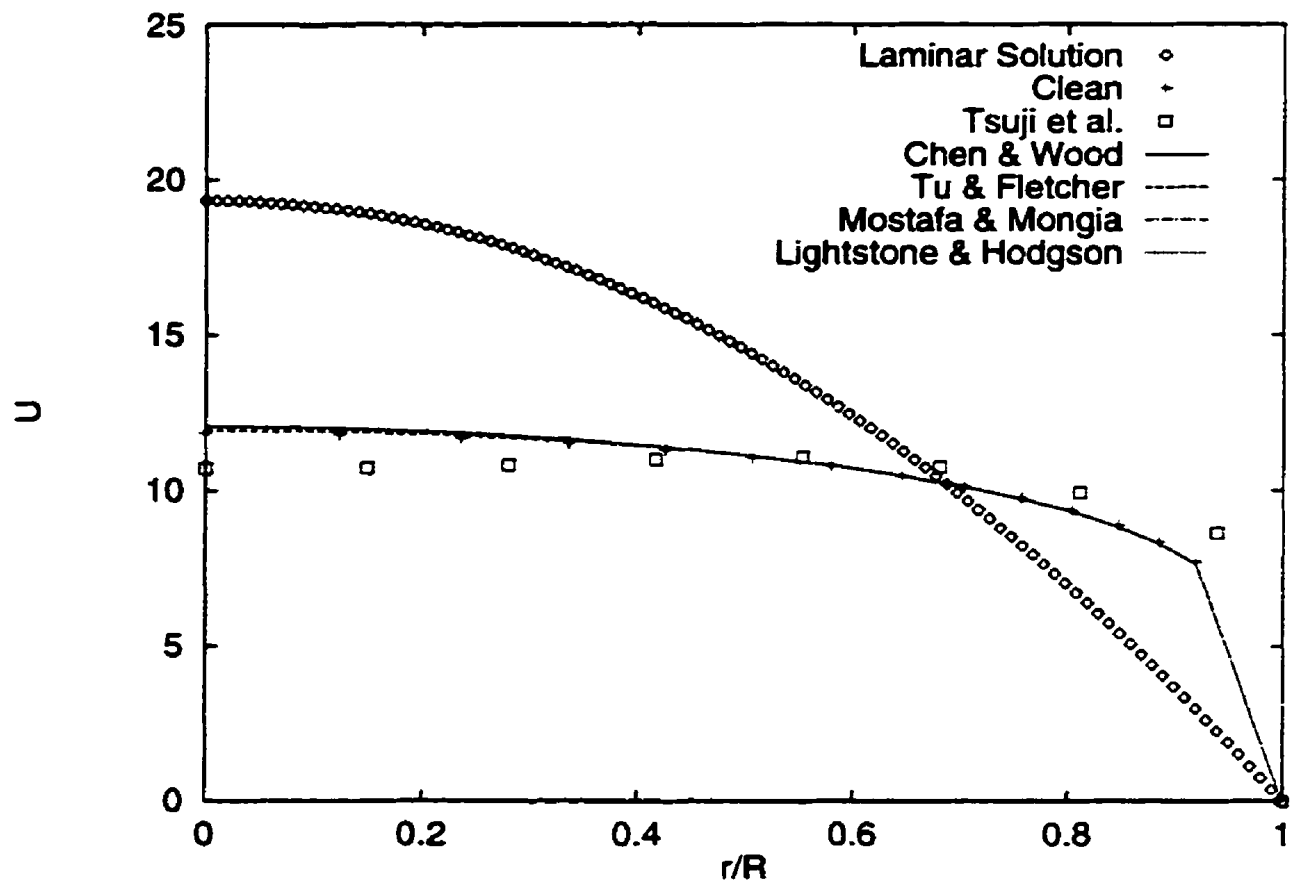

Figure 6.17: Mean velocity: Predictions of experimental data of Tsuji et al[44]. Re $\approx$ $22000, d_{p}=500 \mu \mathrm{m}, w=3.4$ (Case 3)

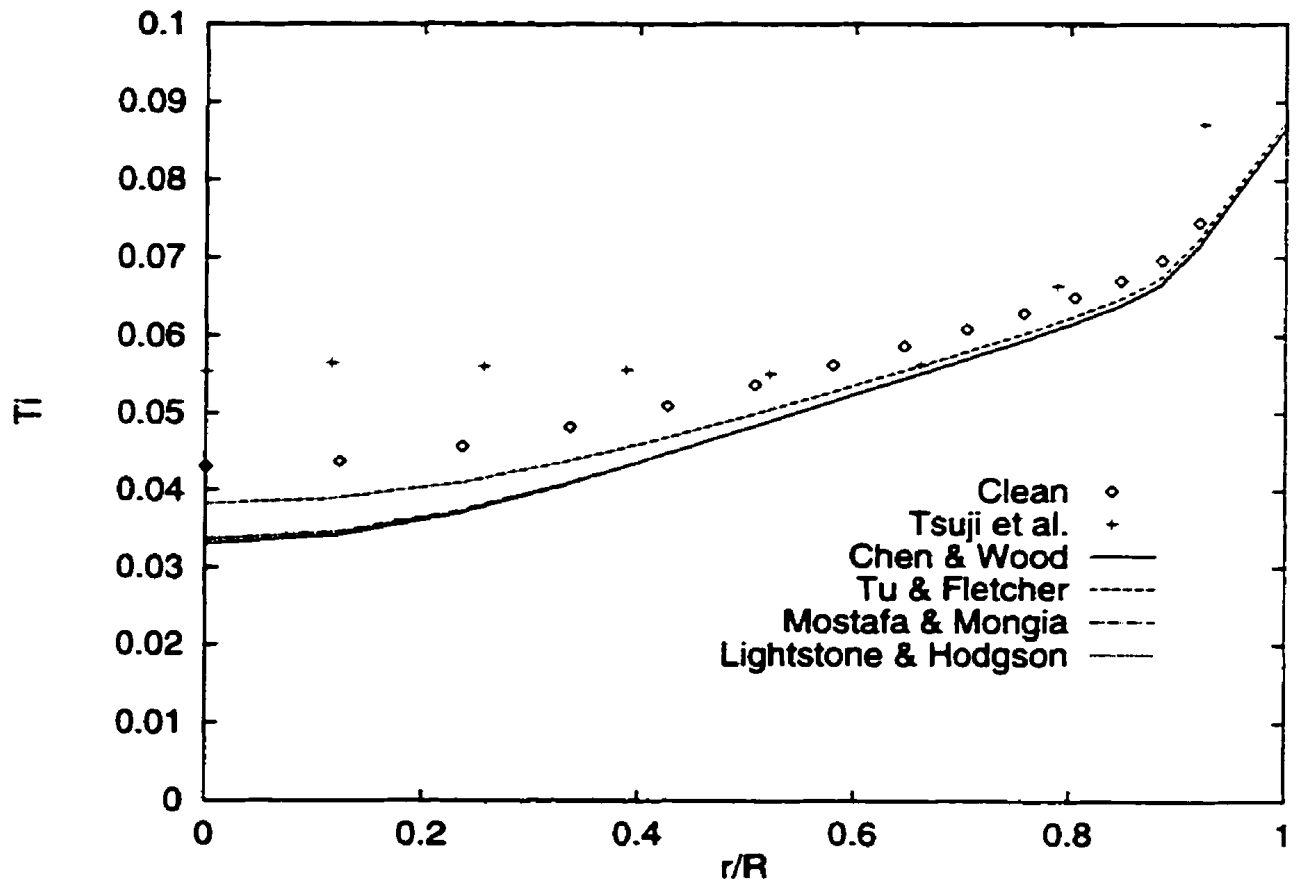

Figure 6.18: Turbulence intensity: Predictions of experimental data of Tsuji et al[ 44$]$. $R e \approx 22000, d_{p}=500 \mu \mathrm{m}, w=1.3$ (Case 3) 


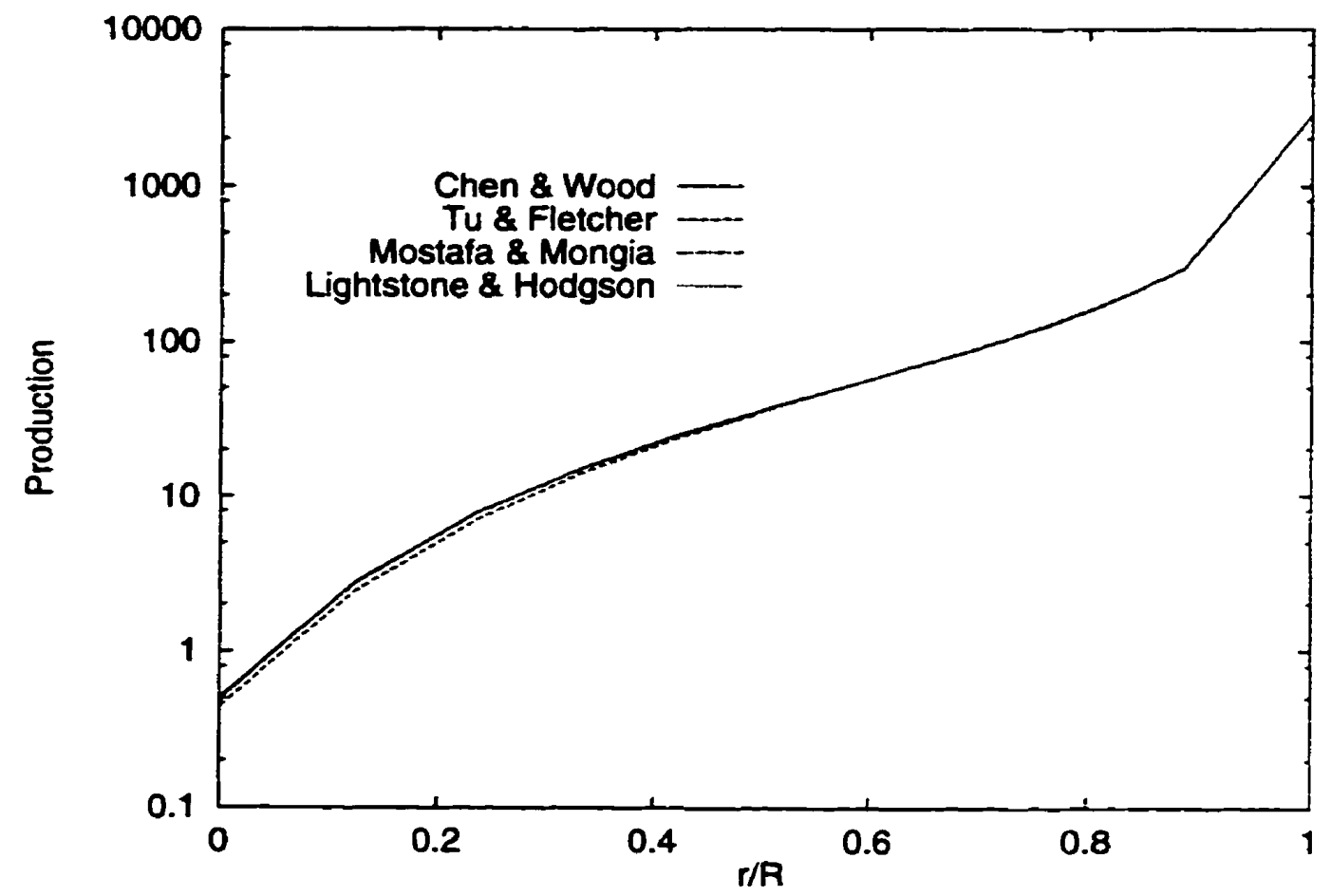

Figure 6.19: Production of $k$ : Case 3

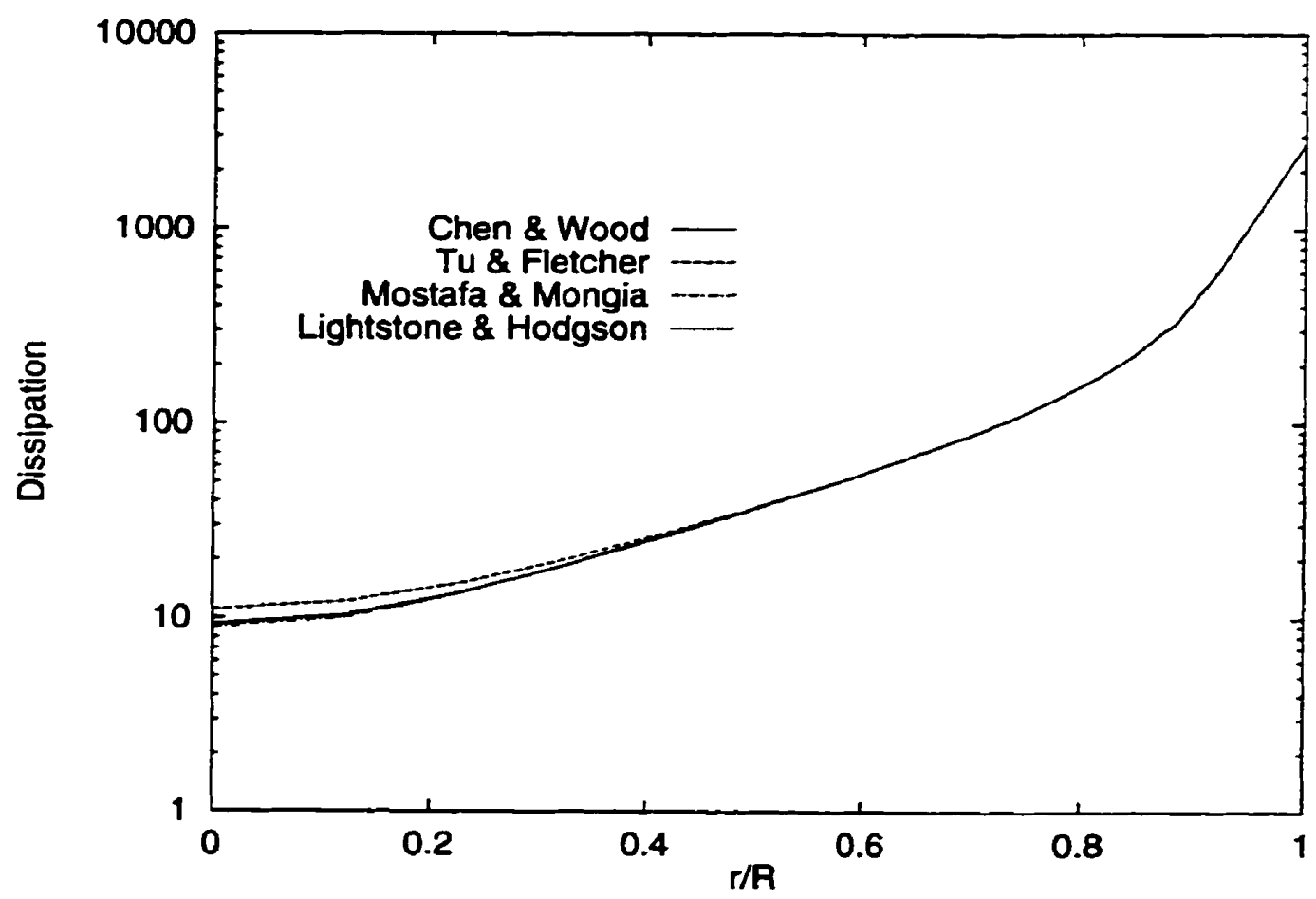

Figure 6.20: Dissipation of $k$ : Case 3 


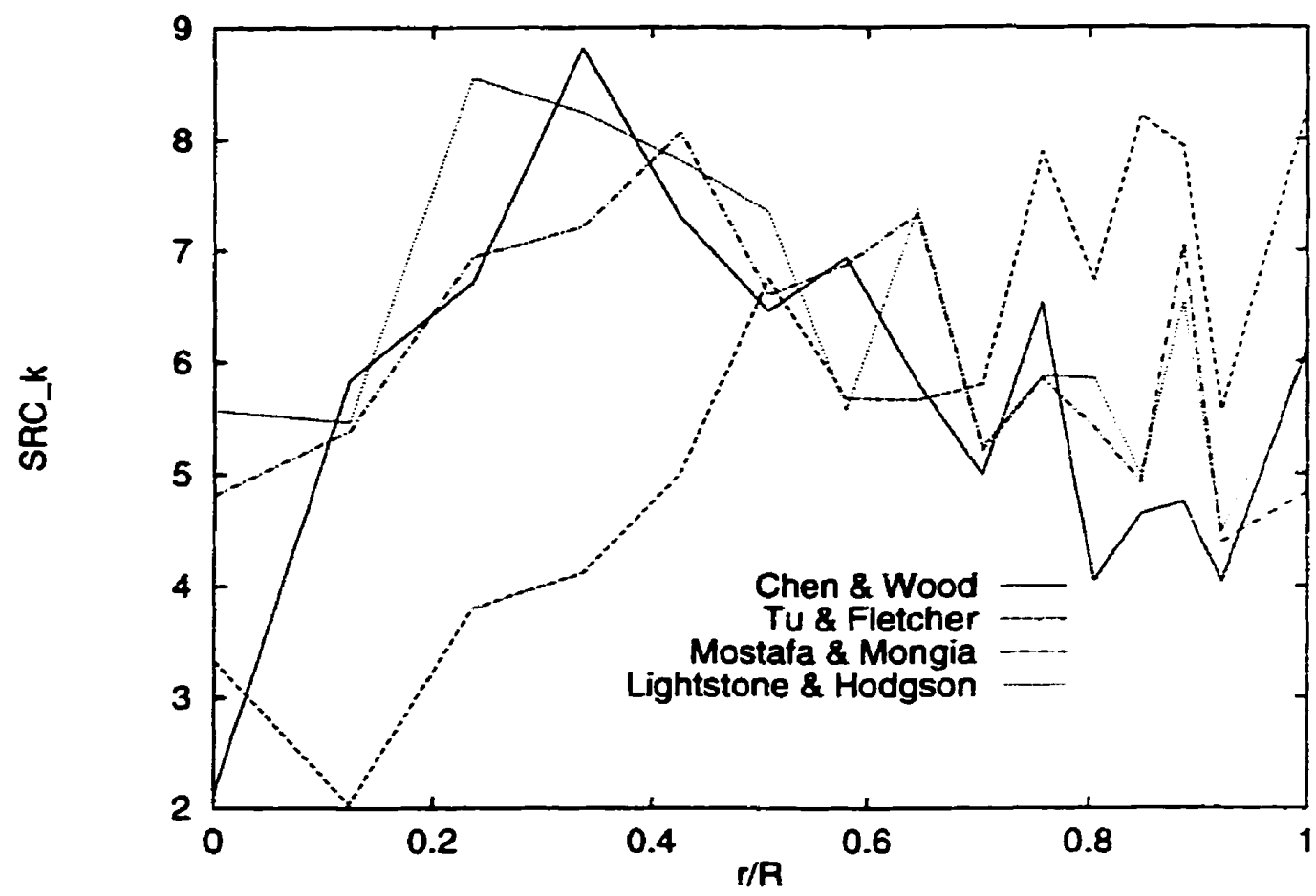

Figure 6.21: Source to $k$ equation, $S_{k}$ : Case 3

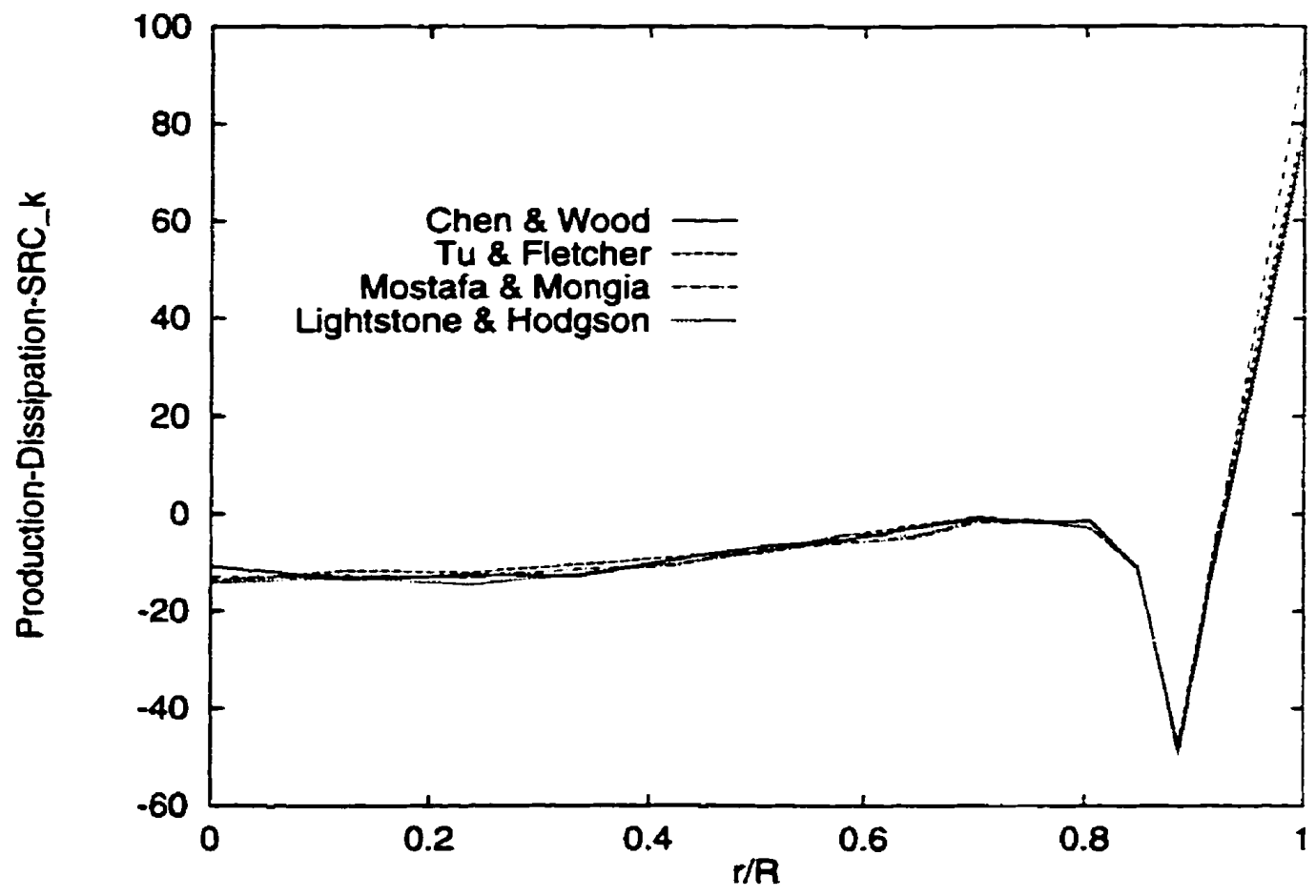

Figure 6.22: Total source to $k$ equation, Production $-\epsilon-S_{k}$ : Case 2 


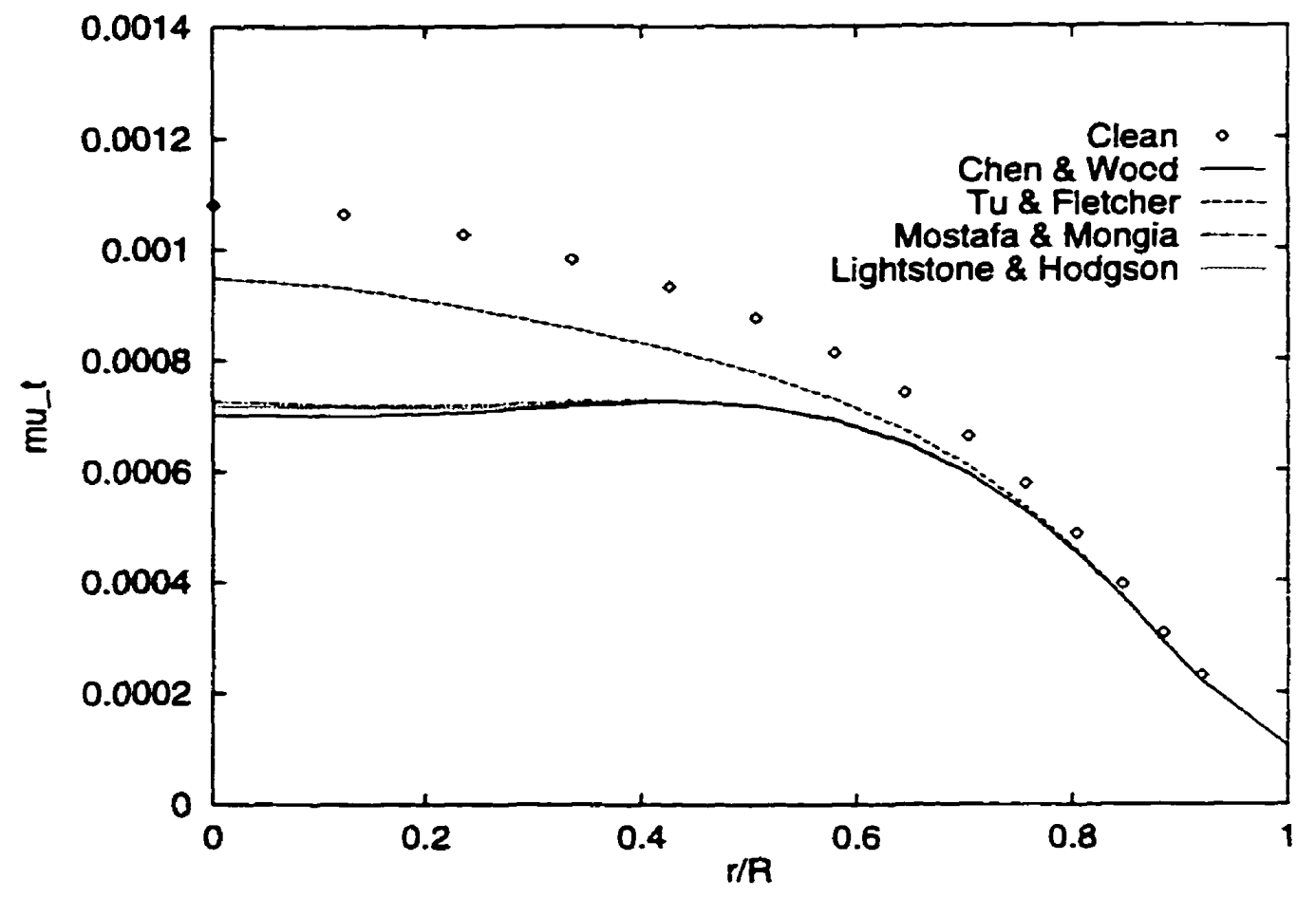

Figure 6.23: Turbulent Viscosity, $\mu_{t}$ : Predictions from the models and clean solution. Case 2.

Notice from the figures that the experimental results of Tsuji et al predict a decrease in turbulence intensity between Case 1 and Case 2 from the centerline to $r / R \approx 0 . \bar{j}$. This is contrary to the expected trend. In order to see an increase in turbulence intensity across the pipe a loading below 1 should be used (see [44]). The models of Chen and Wood, Mostafa and Mongia, and Lightstone and Hodgson produce similar results. An increase in turbulence intensity is seen for both cases across the pipe. This is in agreement with the general experimental trend, however the magnitude of the change is quite far off the experimental results of Tsuji et al. The model of Tu and Fletcher captures neither the experimental trend or the general trend. It is interesting to note that this model was unable to capture the trend for a decrease in the loading ratio, since it was designed specifically to do so.

\subsection{Analysis}

The models of Chen and Wood, Mostafa and Mongia, and Lightstone and Hodgson all yield very similar results. The models are all a function of the particle time constant. $\tau_{p}$. a reflection of the inertia of the particle defined as: 


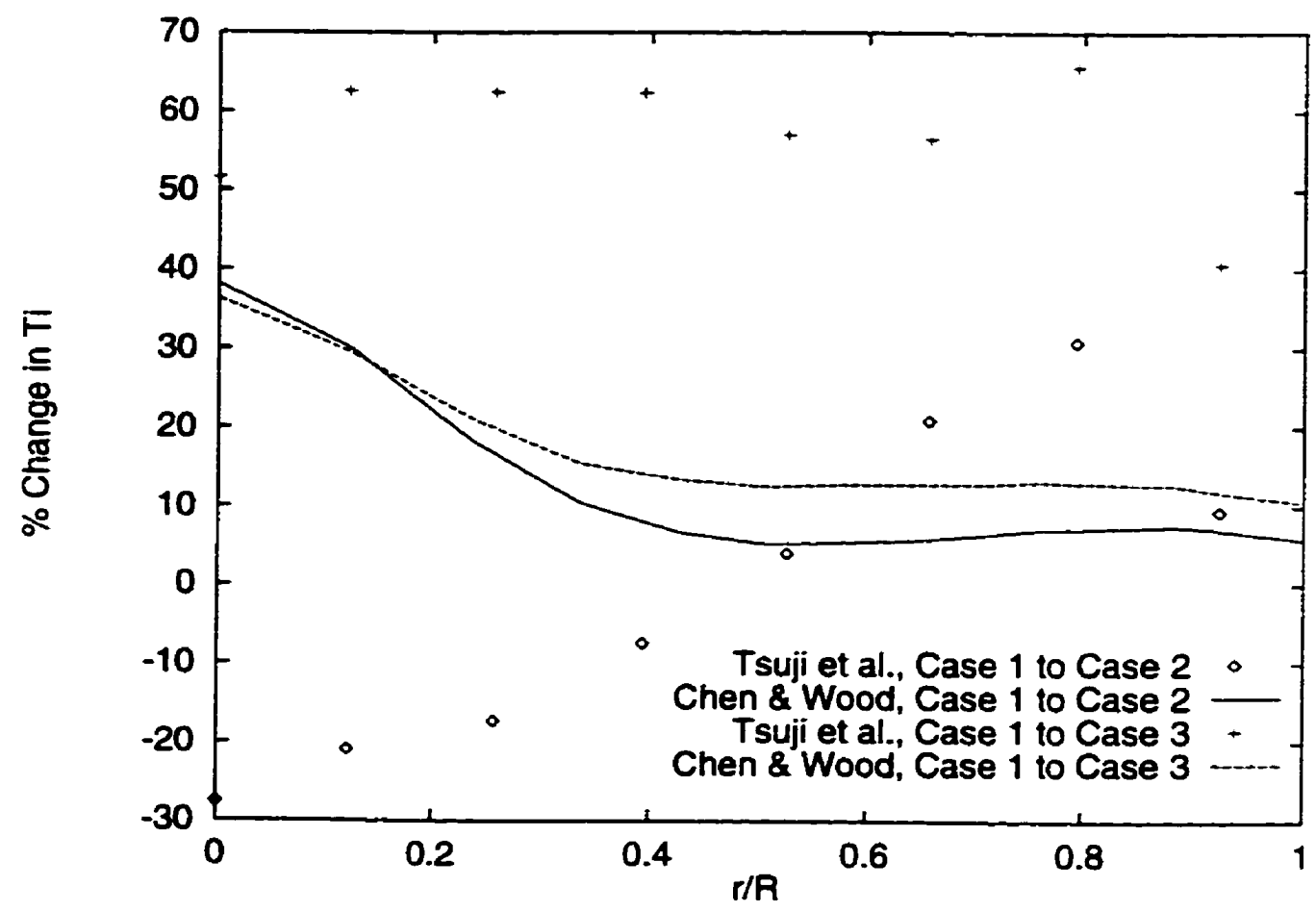

Figure 6.24: Chen and Wood: Prediction of change in turbulence intensity

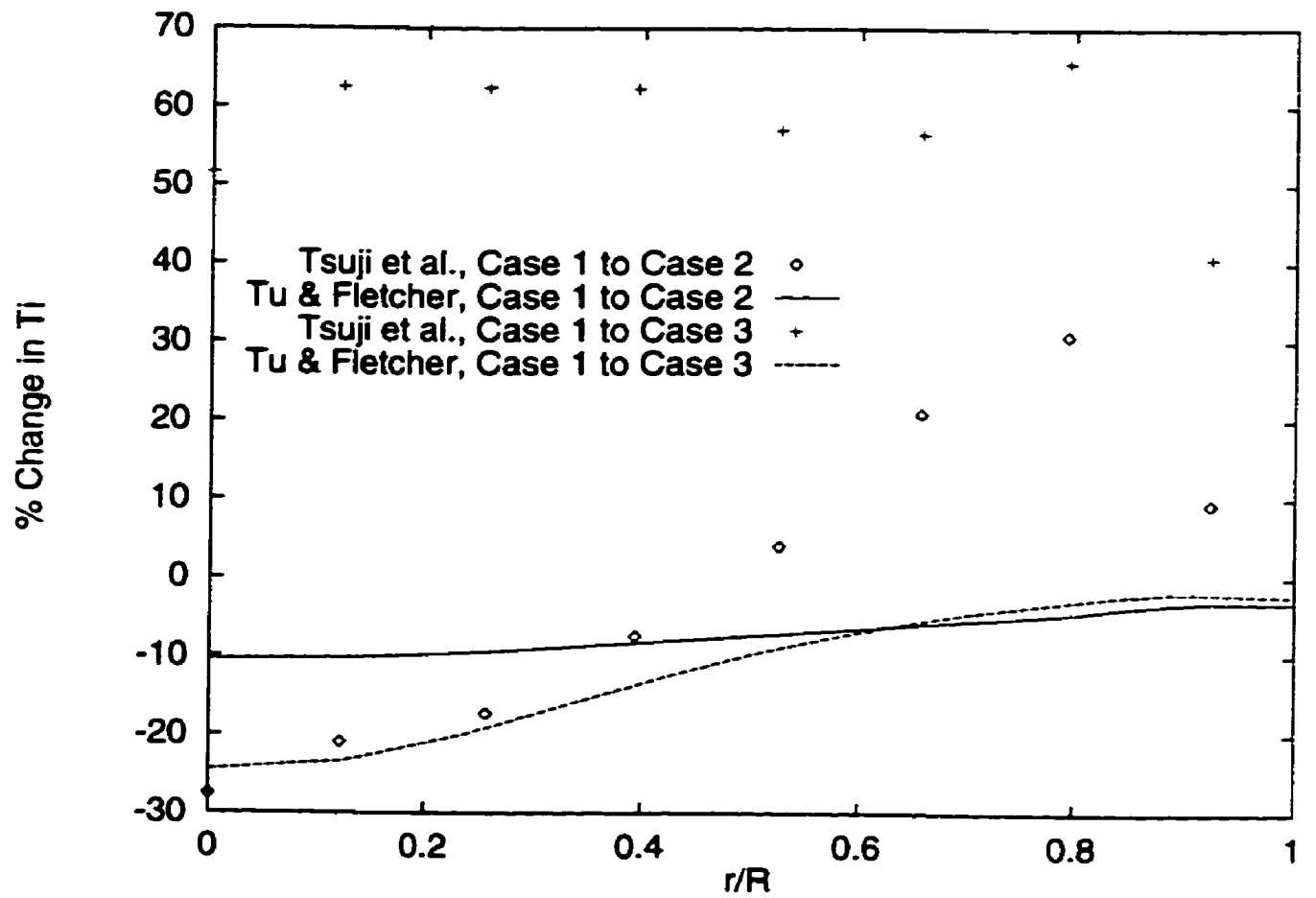

Figure 6.25: Tu and Fletcher: Prediction of change in turbulence intensity 


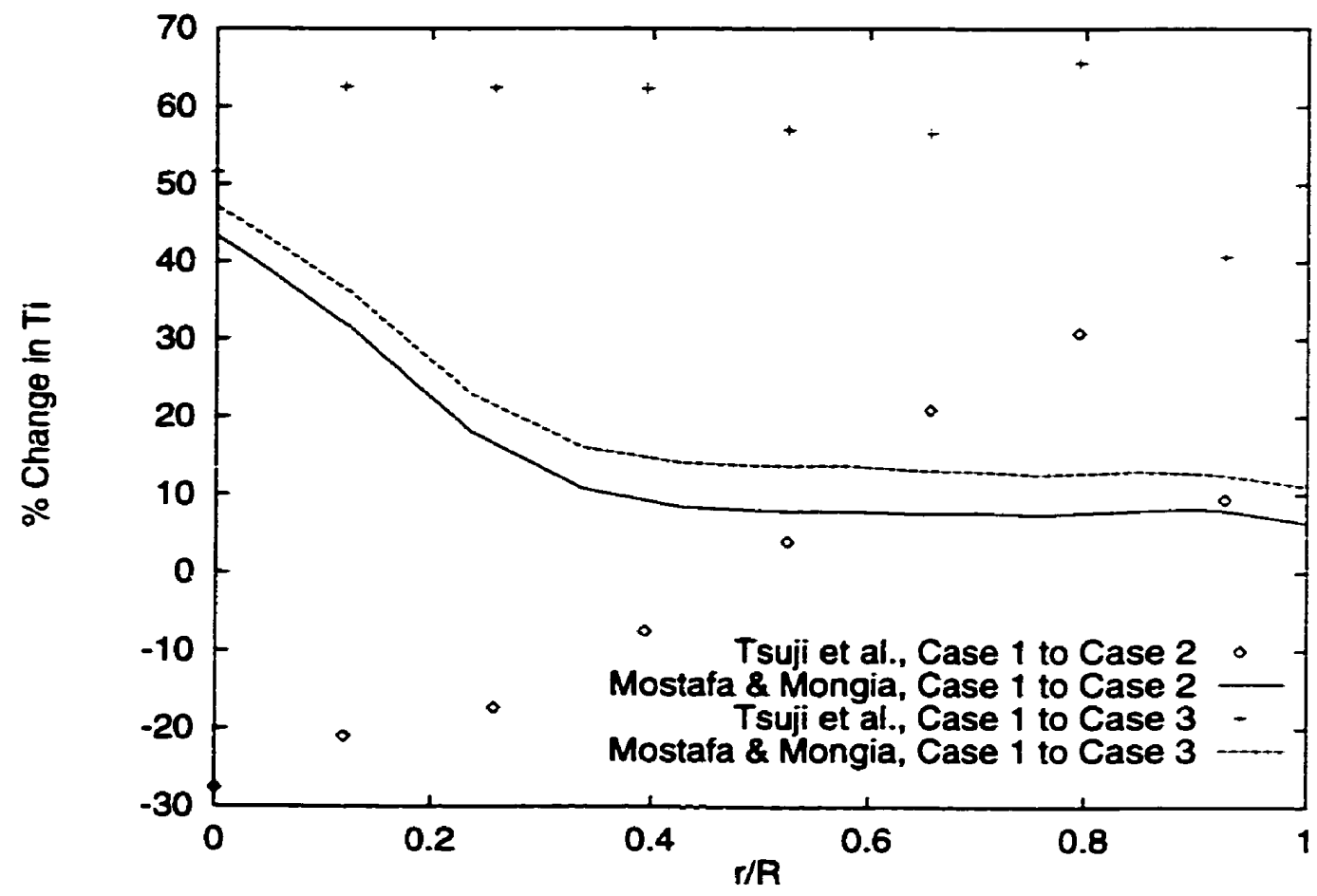

Figure 6.26: Mostafa and Mongia: Prediction of change in turbulence intensity

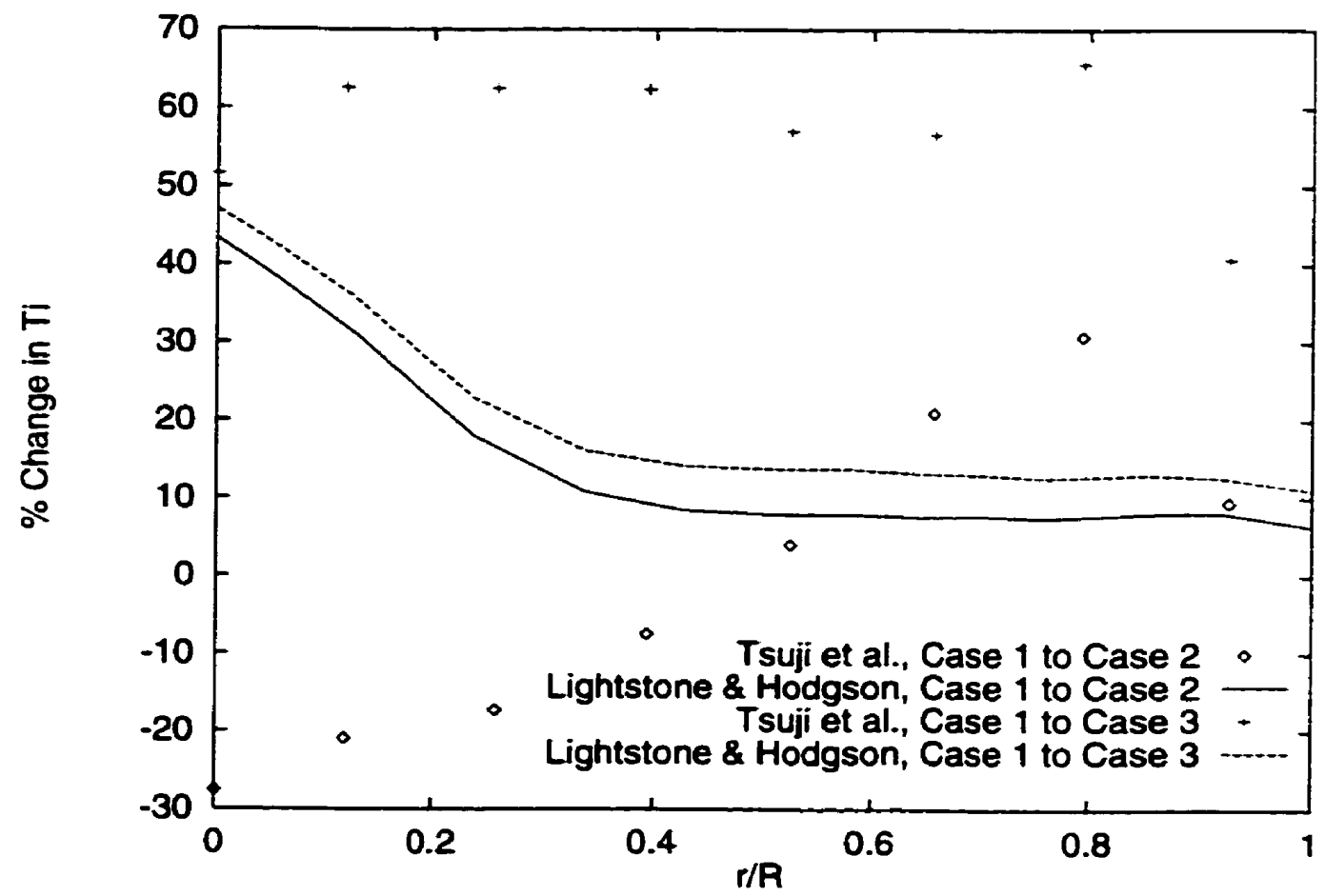

Figure 6.27: Lightstone and Hodgson: Prediction of change in turbulence intensity. 


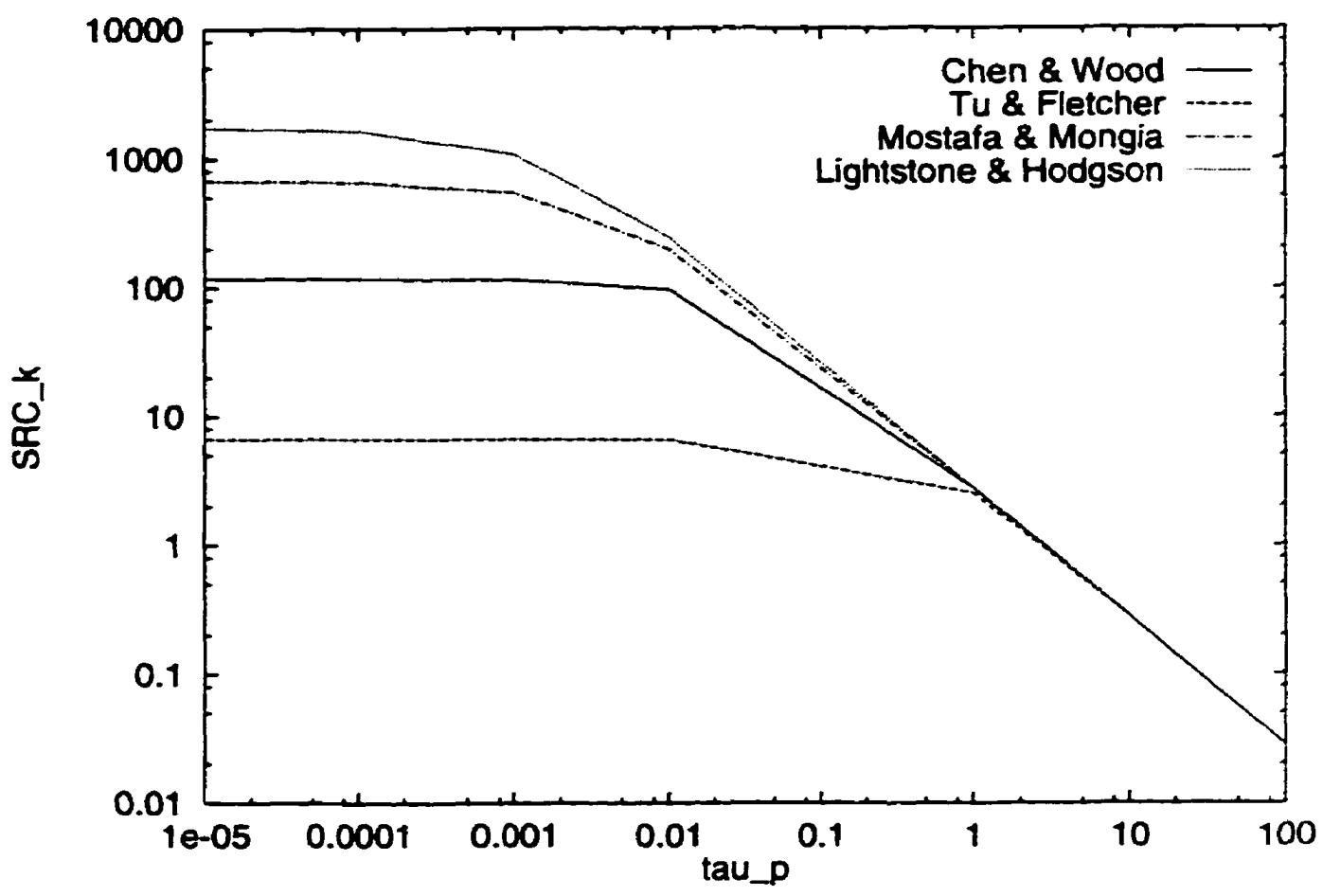

Figure 6.28: $S_{k}$ vs $\tau_{p}$

$$
\tau_{p}=\frac{d^{2} \rho_{p}}{18 \mu}
$$

In order to determine in what range of $\tau_{p}$ the models diverge from each other. the source term, $S_{k}$ for each model was plotted as a function of $\tau_{p}$. This is shown in Figure 6.28. The values for $k, \epsilon$ and $\alpha$ used in this analysis are the same for each model. and are taken from the solution for Case 1 obtained using Chen and Wood's model at $r / R=0 . \overline{3}$. The two values of $\tau_{p}$ used in this investigation were $\tau_{p}=0.12$ and $\tau_{p}=0.78$ for the $200 \mu \mathrm{m}$ and $500 \mu \mathrm{m}$ diameter particles respectively

The models are all of the form:

$$
S_{k}=\frac{2 k \alpha}{\rho_{g} \tau_{p}} f
$$

The dimensionless function $f$ goes to zero as $\tau_{p}$ goes to zero for all models. Howerer. since $\tau_{p}$ appears in the denominator of all the models, as $\tau_{p}$ approaches zero the source term approaches $0 / 0$. Thus the source terms are undefined at $\tau_{p}=0$. At $\tau_{p} \geq 10$ the models all collapse to the same curve. At $\tau_{p} \leq 10$ the model of $\mathrm{Tu}$ and Fletcher diverges from the other models. The model of Chen and Wood is the next to diverge at a value of approximately $\tau_{p} \leq 1$ and reaching a maximum value at $\tau_{p} \leq 0.01$. The model of 
Mostafa and Mongia begins to diverge from that of Lightstone and Hodgson at $\tau_{p} \leq 1$ : the profile begins to flatten out around $\tau_{p} \leq 0.01$. The model of Lightstone and Hodgson has the highest maximum value of any of the four models; it is reached at $\tau_{p}=1 * 10^{-1}$.

The model of Lightstone and Hodgson diverges significantly from the model of Mostafa and Mongia at a value of $\tau_{p}<0.01$. Since the model of Lightstone and Hodgson was formulated to capture the crossing trajectory effect, it is interesting to look at the range of $\tau_{p}$ for which the crossing trajectory effect is significant. The crossing trajectory effect becomes important when the time it take for a particle to cross an eddy, $\tau_{t r}$, is less that the eddy lifetime, $\tau_{e}$. These timescales are defined as:

$$
\begin{aligned}
\tau_{e} & =\frac{l_{e}}{\sqrt{\frac{2}{3} k}} \\
l_{e} & =\frac{C_{\mu}^{3 / 4} k^{3 / 2}}{\epsilon} \\
\tau_{t r} & =-\tau_{p} \ln \left(1-\frac{l_{e}}{\left(\tau_{p}\left|\overrightarrow{u_{g}}-\overrightarrow{u_{p}}\right|\right)}\right)
\end{aligned}
$$

where $C_{\mu}=0.09$.

For the flow considered in this analysis $\tau_{t r}$ is less than $\tau_{e}$ for $\tau_{p} \geq 0.06$ (note that $\left|\overrightarrow{u_{g}}-\overrightarrow{u_{p}}\right|$ was approximated by $\tau_{p} g$ ). In this range the model of Lightstone and Hodgson is similar to the model of Mostafa and Mongia. So for this flow the model of Lightstone and Hodgson does not manage to capture the crossing trajectory effect. This could be attributed to the approximation to the relative velocity, $u_{R E L}=\tau_{p} g$. Further research is required to determine the length of time required for particles in this particular flow to reach their terminal velocity.

\subsection{Summary}

This chapter began with background information: the experiment of Tsuji et al was outlined and details of the numerical simulation presented.

The turbulence modulation models of Chen and Wood [4], Tu and Fletcher [45]. Mostafa and Mongia [30], and Lightstone and Hodgson were studied. Three cases were investigated such that there was one change in particle size and one change in loading ratio between the three cases. Turbulence intensity and mean velocity profiles obtained 
from simulations with each model were compared against experimental data from Tsuji et al.

The models of Chen and Wood, Mostafa and Mongia, and Lightstone and Hodgson gave nearly identical results for all three experimental cases. Analysis performed and this was attributed to the models all collapsing to similar values. The results obtained from Tu and Fletcher differed from the other three models. Each process that contributes to $k$ was examined and it was determined that it is through the turbulent viscosity, $\mu_{t}$, that the differences in the four models appear in the flow solution.

The models of Chen and Wood, Mostafa and Mongia, and Lightstone and Hodgson all predicted the general turbulence modulation trends, but could not reproduce the experimental results. The model of $\mathrm{Tu}$ and Fletcher was unable to reproduce even the general turbulence modulation trends.

Analysis was performed to determine the behaviour of the models with changing $\tau_{p}$. It was found that for values of $\tau_{p}>10$ all the models converge to the same line. Thr model of Lightstone and Hodgson was found to be incapable of capturing the crossing trajectory effect for the flow considered. 


\section{Chapter 7}

\section{Conclusion}

\subsection{Summary and Conclusions}

This thesis investigated the problem of turbulence modulation, the process whereby the fluid-phase turbulence is modified by the presence of particles. Two primary parameters that effect turbulence modulation were identified from experimental data: particle diameter and loading ratio. In general, large particles will cause an increase in turbulence intensity and small particles will cause a decrease in turbulence intensity. Intermediate sized particles will have a mixed effect. For large particles, an increase in the loading ratio serves to further increase the turbulent kinetic energy of the flow. For small particles. as the loading ratio is initially increased there will be a further reduction in turbulence intensity. As the loading ratio is increased even more the turbulence intensity begins to increase.

Four mechanisms were identified as contributing to the turbulence modulation process. These are:

1. Energy transfer through the drag force.

2. Increased dissipation through particle collisions with the small eddies.

3. Energy production through vortex shedding and wake effects.

4. Energy production through the crossing trajectory effect. The particles falling through the eddies will increase the mean velocity gradients and consequently: the turbulent kinetic energy of the flow.

The effect of particles on the gas-phase is incorporated into the momentum. $k$ and $\epsilon$ equations through an additional source term. The source term appearing in the $k$ equation required that the correlation $\overline{u_{g i}^{\prime} u_{p i}^{\prime}}$ be modeled. 
This investigation used the $k-\epsilon$ model of Launder and Spalding [21] to solve the turbulent flow field. The SSF model of Shuen, Chen and Faeth [40] was used to determine the particle trajectories. Three turbulence modulation models were selected as the focus of this work: the model of Chen and Wood [4], the model of Tu and Fletcher [45]. and the model of Mostafa and Mongia [30]. The models are included as sources to the $k-\epsilon$ equation. None of them can predict an increase in turbulence intensity.

The new model of Lightstone and Hodgson accounts for the crossing trajectory effect. whereas the other models do not. It is similar in form to the model of Mostafa and Mongia except the relative velocity between the gas and particle phases is incorporated into one of the timescales.

The models were evaluated using the TASCflow CFD code. Extensive validation was performed to make sure the code worked as it should and to acquire a measure of the accuracy of the predictions for flows which are less complex than those which exhibit turbulence modulation.

The turbulence modulation models were evaluated by comparing the model predictions to the experimental results of Tsuji et al [44]. This experiment involved a gasparticle flow through a vertical pipe. Turbulence intensity and mean velocity measurements were taken at the end of the pipe in the fully developed region. Three experimental cases were selected for this investigation. Case 1 involved $200 \mu \mathrm{m}$ diameter particles at a loading ratio of 3.2. Case 2 involved involved $200 \mu \mathrm{m}$ diameter particles at a loading ratio of 1.3; this case provided a measure of the turbulence modulation models' ability to predict changes in turbulence intensity due to a change in loading ratio. Case 3 involved $500 \mu \mathrm{m}$ diameter particles at a loading ratio of 3.4; this case provided a measure of the turbulence modulation models' ability to predict changes in turbulence intensity due to a change in particle diameter.

The results for Case 1 show that the models of Chen and Wood. Mostafa and Mongia. and Lightstone and Hodgson all give similar results. These models under-predict the experimental turbulence intensity result near the centerline and over-predict the turbulence intensity across the rest of the pipe. The prediction obtained using the model of $\mathrm{Tu}$ and Fletcher exhibits little change from the clean results. It was found that the reason the models of Chen and Wood, Mostafa and Mongia, and Lightstone and Hodgson gave similar results and the model of Tu and Fletcher differed was because the magnitude of the source term of the first three models are within $10 \%$ of each other, while the magnitude of Tu and Fletcher's source term is $90 \%$ lower.

The model of $\mathrm{Tu}$ and Fletcher differs from the other models again for Case 2. In this case all of the models over-predict the turbulence intensity across the pipe. For Case 3 
once again the model of $\mathrm{Tu}$ and Fletcher yields a different solution than the other models. In this case the experimental results are under-predicted by all of the models.

To determine how the models interact with $k-\epsilon$ equation, the expression for turbulent kinetic energy was broken down into components and the production, dissipation. source term to account for turbulence modulation, turbulent viscosity and total source term (production - dissipation - source) examined for each case. It was found that the diffusion term, specifically $\mu_{t}$, plays an important role in modifying the turbulence intensityprofiles.

The effectiveness of the models at predicting general experimental trends was also examined. The percent change in turbulence intensity between Case 1 and Case 2 (change in loading ratio), and Case 1 and Case 3 (change in particle diameter) was calculated for the experimental results and model predictions. It was found that the models of Chen and Wood, Mostafa and Mongia, and Lightstone and Hodgson all predicted the correct general experimental trends, however they could not reproduce the experimental results of Tsuji et al. The model of Tu and Fletcher was not able to reproduce either the general experimental trends or the results of Tsuji et al. This is an interesting result since the model of Tu and Fletcher was designed specifically to capture the effect of a change in loading ratio.

In order to determine the behaviour of the models with changing particle response time, $\tau_{p}$, an analysis was performed. It was found that for values of $\tau_{p}>10$ all the models converged to the same curve. The model of Lightstone and Hodgson was found to be incapable of capturing the crossing trajectory effect for the flow considered.

\subsection{Recommendations for Future Work}

There are many factors which affect turbulence modulation, making it a very complex problem. This thesis has managed only to scratch the surface of the processes and problems involved in modeling turbulence modulation. Suggestions for future work in this area include:

- Vortex shedding and wake effects should be studied in the context of turbulence production. A model that accounts for these effects and can be incorporated into the $k-\epsilon$ equation is required.

- The impact of the source term appearing in the $\epsilon$ equation on the turbulence intensity should be studied. A model for $S_{\epsilon}$ that is independent of the model for $S_{k}$ should be developed. 
- The performance of the model of Lightstone and Hodgson under different flow conditions and configurations should be investigated. The effect of using the terminal velocity of the particle as an approximation to the relative velocity should also be studied.

- The impact of the constant appearing in the models of Chen and Wood and Tu and Fletcher should be investigated. Alternative methods of incorporating a pararneter that represents a change in loading ratio should be explored.

- As mentioned briefly at the beginning of this thesis, in the Lagrangian formulation the values for $u_{g i}^{\prime}$ and $u_{p i}^{\prime}$ are coarsely known. These coarse values can be used to evaluate the correlation $\overline{u_{g i}^{\prime} u_{p i}^{\prime}}$ and it therefore would not require modeling. Research is required into the development of a numerical procedure which can provicle the bookkeeping for these values. 


\section{Appendix A}

\section{Derivation of Turbulent Kinetic Energy Equation}

An equation for the turbulent kinetic energy of the gas-phase, including the effect of the particulate phase on the gas phase is obtained by subtracting the expression for the mean kinetic energy from the expression for the turbulent kinetic energy. Incompressible. Stokian, constant viscosity flow is assumed throughout.

\section{Mean Kinetic Energy}

An expression for the mean kinetic energy is obtained by multiplying the equation for mean momentum, Eq.(2.11), by $U_{g i}$ and summing over $i$. Neglecting body forces this yields the following equation:

$$
\begin{aligned}
\rho_{g} \frac{\partial K}{\partial t}+\rho_{g} U_{g j} \frac{\partial K}{\partial x_{j}}= & -\frac{\partial U_{g i} \bar{P}}{\partial x_{i}}+\rho_{g} \overline{u_{g i}^{\prime} u_{g j}^{\prime}} \frac{\partial U_{g i}}{\partial x_{j}}-\rho_{g} \frac{\partial}{\partial x_{j}} U_{g i} \overline{u_{g i}^{\prime} u_{g j}^{\prime}} \\
& +\mu \frac{\partial}{\partial x_{i}} U_{g j}\left(\frac{\partial U_{g i}}{\partial x_{j}}+\frac{\partial U_{g j}}{\partial x_{i}}\right) \\
& -\frac{\mu}{2}\left(\frac{\partial U_{g i}}{\partial x_{j}}+\frac{\partial U_{g j}}{\partial x_{i}}\right)^{2}-U_{g i} \overline{F_{p i}}
\end{aligned}
$$

where $\mathrm{K}$ is the mean kinetic energy per unit mass, equal to $\frac{1}{2} U_{g i} U_{g i}$. 


\section{Total Kinetic Energy}

Neglecting body forces, start with the instantaneous momentum equation. Eq. (2.9). multiply by $u_{g i}$, and sum over $i$ to obtain the instantaneous kinetic energy equation:

$$
\rho_{g} \frac{D \frac{1}{2} u_{g i}^{2}}{D t}=-U_{g i} \frac{\partial P}{\partial x_{i}}+\mu \frac{\partial}{\partial x_{j}} u_{g i}\left(\frac{\partial u_{g i}}{\partial x_{j}}+\frac{\partial u_{g j}}{\partial x_{i}}\right)-\frac{1}{2} \mu\left(\frac{\partial u_{g i}}{\partial x_{j}}+\frac{\partial u_{j}}{\partial x_{i}}\right)^{2}-u_{g i} F_{p i}
$$

Applying Reynolds decomposition to Eq. (A) and time-averaging vields the equation for the total kinetic energy of the gas phase:

$$
\begin{aligned}
\rho_{g}\left(\frac{\partial K}{\partial t}+U_{g j} \frac{\partial K}{\partial x_{j}}+\frac{\partial k}{\partial t}+U_{g j} \frac{\partial k}{\partial x_{j}}\right) & =-u_{g i} \frac{\partial \bar{P}}{\partial x_{i}}-\rho_{g} \frac{\partial}{\partial x_{i}} \overline{u_{g i}^{\prime}\left(\frac{P^{\prime}}{\rho_{g}}+k\right)} \\
& -\rho_{g} \frac{\partial}{\partial x_{i}} U_{g j} \overline{u_{g i}^{\prime} u_{g j}^{\prime}}+\mu \frac{\partial}{\partial x_{i}} U_{g j}\left(\frac{\partial U_{g i}}{\partial x_{j}}+\frac{\partial L_{g j}^{\prime}}{\partial x_{i}}\right) \\
& +\mu \frac{\partial}{\partial x_{i}} \overline{u_{g j}^{\prime}\left(\frac{\partial u_{g i}^{\prime}}{\partial x_{j}}+\frac{\partial u_{g j}^{\prime}}{\partial x_{i}}\right)} \\
& -\frac{\mu}{2}\left(\frac{\partial U_{g i}}{\partial x_{j}}+\frac{\partial U_{g j}}{\partial x_{i}}\right)^{2}-\frac{\mu}{2}\left(\frac{\partial u_{g i}^{\prime}}{\partial x_{j}}+\frac{\partial u_{g j}^{\prime}}{\partial x_{i}}\right)^{2} \\
& -\left(U_{g i} \overline{F_{p i}}-\overline{u_{i}^{\prime} F_{p i}^{\prime}}\right)
\end{aligned}
$$

where $\mathrm{k}$ is the turbulent kinetic energy per unit mass equal to $\overline{\frac{1}{2} u_{g i}^{\prime} u_{g i}^{\prime}}$.

\section{Turbulent Kinetic Energy}

The turbulent kinetic energy is obtained from subtracting equation (A.1) from equation (A.2):

$$
\begin{aligned}
\rho_{g} \frac{\partial k}{\partial t}+U_{g j} \frac{\partial k}{\partial x_{j}} & =-\rho_{g} \overline{u_{g i}^{\prime} u_{g j}^{\prime}} \frac{\partial U_{g j}}{\partial x_{i}}-\rho_{g} \frac{\partial}{\partial x_{i}} \overline{u_{g i}^{\prime}\left(\frac{P^{\prime}}{\rho_{g}}+k\right)} \\
& +\mu \frac{\partial}{\partial x_{i}} \overline{u_{g j}^{\prime}\left(\frac{\partial u_{g i}^{\prime}}{\partial x_{j}}+\frac{\partial u_{g j}^{\prime}}{\partial x_{i}}\right)}-\frac{\mu}{2} \overline{\left(\frac{\partial u_{g i}^{\prime}}{\partial x_{j}}+\frac{\partial u_{g j}^{\prime}}{\partial x_{i}}\right)}-\overline{u_{g i}^{\prime} F_{p i}^{\prime}}
\end{aligned}
$$




\section{Appendix B}

\section{Analysis of Momentum Source Term}

As discussed in Section 4.4 the source term to the mean momentum equation is obtained by substituting the analytical solution to the particle equation of motion into the expression for the instantaneous particle source term, $F_{p}$, and time-averaging. This gires the following expression for the influence of the particles on the mean momentum:

$$
-\overline{F_{p}}=\underbrace{\frac{\alpha}{\delta t}\left(u_{p}^{o}-u_{g}\right)\left(1-\exp \left(\frac{-\delta t}{\tau_{p}}\right)\right)}_{I}-\underbrace{\left[\alpha g+\alpha g \frac{\tau_{p}}{\delta t}\left(\exp \left(\frac{-\delta t}{\tau_{p}}\right)-1\right)\right]}_{I I}
$$

where Term I represents the effect of the velocity difference between the gas and particle phases on the mean momentum while Term II is the effect of the interaction of the particle and gravity on the mean momentum. The following analysis shows that Term II is negligible.

To begin, perform a Taylor series expansion (up to the second order term) of Term II:

$$
\begin{aligned}
\alpha g & +\alpha g \frac{\tau_{p}}{\delta t}\left(\exp \left(\frac{-\delta t}{\tau_{p}}\right)-1\right) \\
& =\alpha g+\alpha g \frac{\tau_{p}}{\delta t}\left(1-\frac{\delta t}{\tau_{p}}+\frac{1}{2} \frac{\delta t^{2}}{\tau_{p}^{2}}-1\right) \\
& =\frac{\alpha g}{2} \frac{\delta t}{\tau_{p}}
\end{aligned}
$$

So as $\delta t \rightarrow 0$ Term II vanishes.

Next a Taylor series expansion is applied to Term I:

$$
\frac{\alpha}{\delta t}\left(u_{p}^{o}-u_{g}\right)\left(1-\exp \left(\frac{-\delta t}{\tau_{p}}\right)\right)=\frac{\alpha}{\delta t}\left(u_{p}^{o}-u_{g}\right)\left(1-1+\frac{\delta t}{\tau_{p}}-\frac{1}{2} \frac{\delta t^{2}}{\tau_{p}^{2}}\right)
$$




$$
=\frac{\alpha\left(u_{p}^{o}-u_{g}\right)}{\tau_{p}}-\frac{\alpha\left(u_{p}^{o}-u_{g}\right)}{2 \tau_{p}}\left(\frac{\delta t}{\tau_{p}}\right)
$$

As $\delta t \rightarrow 0$ Term I reduces to:

$$
\frac{\alpha}{\delta t}\left(u_{p}^{o}-u_{g}\right)\left(1-\exp \left(\frac{-\delta t}{\tau_{p}}\right)\right) \approx \frac{\alpha\left(u_{p}^{o}-u_{g}\right)}{\tau_{p}}
$$

So as $\delta t \rightarrow 0$ Term I dominates.

Now look at the case where $\tau_{p}$ is large, that is, the particles are heavy and do not exactly follow the fluid. At their limit the particles will be at their terminal velocity where,

$$
u_{p}=u_{g}-\tau_{p} g
$$

Substituting this in to Term I and Term II yields:

$$
\begin{aligned}
& \frac{\alpha}{\delta t}\left(u_{g}-\tau_{p} g-u_{g}\right)\left(1-\exp \left(\frac{-\delta t}{\tau_{p}}\right)\right)-\left[\alpha g+\alpha g \frac{\tau_{p}}{\delta t}\left(\exp \left(\frac{-\delta t}{\tau_{p}}\right)-1\right)\right] \\
= & -\alpha g
\end{aligned}
$$

Substituting the Equation (B.5) into the expanded form of Term I, Eq.(B.4):

$$
\frac{\alpha\left(u_{g}-\tau_{p} g-u_{g}\right)}{\tau_{p}}=-\alpha g
$$

So for flows involving particles with large $\tau_{p}$ Term II does not contribute to the source term. It can therefore be concluded that for $\delta t / \tau_{p} \ll 1$ the influence of Term II is negligible.

Thus, the source term that is used in TASCflow to account for the interaction between the particles and the mean momentum is given by;

$$
-\overline{F_{p}}=\frac{\alpha}{\delta t}\left(u_{p}^{o}-u_{g}\right)\left(1-\exp \left(\frac{-\delta t}{\tau_{p}}\right)\right)
$$




\section{Bibliography}

[1] A. Adeniji-Fashola and C.P. Chen. Modeling of confined turbulent fluid-particle flows using Eulerain and Lagrangian schemes. International Journal of Heat and Mass Transfer, 33:691-701, 1990.

[2] Advanced Scientific Computing Ltd., Waterloo, Ontario, Canada. TASCflow:3D. Version 2.4, Theory Documentation, Volume 1, March 1995.

[3] Advanced Scientific computing Ltd., Waterloo, Ontario, Canada. TASCflow $3 D$. Version 2.4, Theory Documentation, Volume 2, March 1995.

[4] C.P. Chen and P.E. Wood. Turbulence closure model for dilute gas-particle flows. Canadian Journal of Chemical Engineering, 63:349-360, 1985.

[5] P.P. Chen and C.T. Crowe. On the Monte-Carlo method for modelling particle dispersion in turbulence. In Proceedings of International Symposium on Gas-Solid Flow, pages 37-41, New Orleans, LA, 1984.

[6] C.T. Crowe. Review - numerical models for dilute gas-particle flows. Journal of Fluids Engineering, 104:297-303, 1982.

[7] C.T. Crowe. The state-of-the-art in the development of numerical models for dispersed phase flows. In Proceedings of the International Conference on Multiphase. Flows '91 - Tsukuba, pages 49-59, Tsukuba, Japan, September 1991.

[8] C.T. Crowe. Modeling turbulence in multiphase flows. In W. Rodi and F. Martelli. editors, Engineering Turbulence Modelling and Experiments 2, pages 899-913. Elsevier Science Publishers, 1993.

[9] C.T. Crowe, M.P. Sharma, and D.E Stock. The particle-source-in-cell method for gas droplet flow. Journal of Fluids Engineering, 99:325-332, 1977.

[10] G.T. Csanaday. Turbulent diffusion of heavy particles in the atmosphere. Journal of the ATmospheric Sciences, 20:201-208, 1963. 
[11] S. Elghobashi. On predicting particle-laden turbulent flows. Applied Scientific Research, 52:309-329, 1994.

[12] J. Fan, X. Zhang, L. Cheng, and K. Cen. Numerical simulation and experimental study of two-phase flow in a vertical pipe. Aerosol Science and Technology, 27:281291, 1997.

[13] R.W. Fox and A.T. McDonald. Introduction to Fluid Mechanics. John Wiley and Sons, third edition edition, 1985.

[14] R.A. Gore and C.T. Crowe. Effect of particle size on modulating turbulent intensity: International Journal of Multiphase Flow, 15:279-285, 1989.

[15] A.D. Gosman and E. Ioannides. Aspects of computer simulation of liquid-fueled combusters. Journal of Energy, 7:482-490, 1983.

[16] E.A. Hennick. A comparison of stochastic separated flow models for particle dispersion in turbulent flows. Master's thesis, University of Toronto, 1998.

[17] G. Hetsroni. Particles-turbulence interaction. International Journal of Multiphase. Flow, 15(5):735-746, 1989.

[18] J.O. Hinze. Turbulence. McGraw-Hill, second edition, 1975.

[19] R.G. Huget. The Evaluation and Development of Approximation Schemes for the Finite Volume Method. PhD thesis, University of Waterloo, 1985.

[20] C.T. Kenning, V.M.and Crowe. On the effect of particles on carrier phase turbulence in gas-particle flow. International Journal of Multiphase Flow, 23:403-408. 1997.

[21] B.E. Launder and D.B. Spalding. The numerical computation of turbulent flows. Computer Methods in Applied Mechanics and Engineering, 3(269-289), 19ז4.

[22] S.L. Lee and F. Durst. On the motion of particles in turbulent duct flows. International Journal of Multiphase Flow, 8:125-146, 1982.

[23] Y. Levy and F.C. Lockwood. Velocity measurements in a particle laden turbulent free jet. Combustion and Flame, 40:333-339, 1981.

[24] M.F. Lightstone. A Stochastic Model of the Dispersion of Solid Particles in a Turbulent Gaseous Environment. PhD thesis, University of Waterloo, 1992. 
[25] M.F. Lightstone and G.D. Raithby. The validity of the gradient diffusion approach for modelling particle dispersion in a turbulent gaseous flow. In Proceeding of the Fourth Annual Conference of the CFD Society of Canada, pages 473-4i9. 1996.

[26] J.N. Lillington. A vector upstream differencing scheme for problems in fluid flow involving significant source terms in steady-state linear systems. International Journal of Numerical Methods in Fluids, 1:3-16, 1981.

[27] R. Martinuzzi and A. Pollard. Comparative study of turbulence models in predicting turbulent pipe flow. Part I: Algebraic stress and $k-\epsilon$ models. AIAA Journal. $27(1): 29-36,1989$.

[28] D. Modarress, H. Tan, and S. Elghobashi. Two-component LDA measurement in a two-phase turbulent jet. AIAA Journal, 22:396-404, 1984.

[29] D. Modarress, J. Wuerer, and S. Elghobashi. An experimental study of a turbulent round two-phase jet. Chemical Engineering Communications, 28:341-354. 1984.

[30] A.A. Mostafa and H.C. Mongia. On the interaction of particles and turbulent fluid flow. International Journal of Heat and Mass Transfer, 31:2063-2075, 1988.

[31] A.A. Mostafa, H.C. Mongia, V.G. McDonell, and G.S. Samuelsen. Erolution of particle-laden jet flows: A theoretical and experimental study. AIAA Journal. 27:167-183, 1989.

[32] S.V. Patankar. Numerical Heat Transfer and Fluid Flow. Taylor and Francis. 1980.

[33] M.C. Potter and D.C. Wiggert. Mechanics of Fluids. Prentice-Hall, Inc., 1991.

[34] G.D. Raithby. Skew upstream difference schemes for problems involving fluid flow: Computational Methods in Applied Mechanical Engineering, 9:153-164, $19 \overline{\mathbf{r}} 6$.

[35] M.J. Raw. A New Control-Volume-Based Finite Element Procedure for the Nitmerical Solution of the Fluid Flow and Scalar Transport Equations. PhD thesis. University of Waterloo, 1985.

[36] H. Schlichting. Boundary Layer Theory. McGraw-Hill, 6th edition, 1968.

[37] G.E. Schneider and M.J. Raw. A skewed, positive influence coefficient upwinding procedure for control-volume-based finite element convection-diffusion computation. Numerical Heat Transfer, 8:1-26, 1986. 
[38] G.E. Schneider and M.J. Raw. Control-volume finite element method for heat transfer and fluid flow using co-located variable - 1. computational procedure. Numerical Heat Transfer, 11:363-390, 1987.

[39] J.S. Shirolkar, C.F.M Coimbra, and M. Queiroz McQuay. Fundamental aspects of modeling turbulent particle dispersion in dilute flows. Progress in Energy Combustion Science, 22:363-399, 1996.

[40] J.S. Shuen, A.S.P. Solomon, Q.F. Zhang, and G.M. Faeth. A theoretical and experimental study of turbulent particle-laden jets. Technical Report NASA-CR-168-29:3. NASA - Lewis Research Center, 1983.

[41] J.S. Shuen, A.S.P. Solomon, Q.F. Zhang, and G.M. Faeth. Structure of particle-laden jets: Measurements and predictions. AIAA Journal, 23(3):396-404, 1985.

[42] G.I. Taylor. Diffusion by continuous movements. Proceedings of the London Mathematical Society, 20:196-211, 1921.

[43] Y. Tsuji and Y. Morikawa. LDV measurements of an air-solid two-phase flow in a horizontal pipe. Journal of Fluid Mechanics, 120:385-409, 1982.

[44] Y. Tsuji, Y. Morikawa, and H. Shiomi. LDV measurements of an air-solid two-phase flow in a vertical pipe. Journal of Fluid Mechanics, 139:417-434, 1984.

[45] J.Y. Tu and C.A.J. Fletcher. An improved model for particulate turbulence modulation in confined two-phase flows. International Communications in Heat and Mass Transfer, 21:775-783, 1994.

[46] J.S. Vames and T.J. Hanratty. Turbulent dispersion of droplets for air flow in a pipe. Experiments in Fluids, 6:94-104, 1988.

[47] Z. Yuan and E.E. Michaelides. Turbulence modulation in particulate flows - a theoretical approach. International Journal of Multiphase Flow, 18:779-785, 1992.

[48] Q. Zhou and M.A. Leschziner. A time-correlated stochastic model for particle dispersion in anisotropic turbulence. In 8th Turbulent Shear Flows Symposium. 1991.

[49] Q. Zhou and M.A. Leschziner. A Lagrangian particle dispersion model based on a time-correlated stochastic approach. In Proc. 1st Joint JSME-ASME Fluids Engineering Conf., 4th Symp. on Gas-Solid Flow. ASME/JSME, June 1991. 